Aus der Klinik für Unfallchirurgie, Orthopädie und Plastische Chirurgie

(Prof. Dr. med. W. Lehmann)

der Medizinischen Fakultät der Universität Göttingen

Der roboterassistierte

Lymphknotentransfer mittels

vaskularisiertem Omentumlappen in der

\title{
Therapie des sekundären Lymphödems
}

der Extremitäten

\author{
INAUGURAL-DISSERTATION \\ zur Erlangung des Doktorgrades \\ der Medizinischen Fakultät der \\ Georg-August-Universität zu Göttingen
}

vorgelegt von

Dominik Johannes Behringer

aus

Breisach am Rhein

Göttingen 2021 
Dekan:

\section{Betreuungsausschuss}

Betreuer/in

Ko-Betreuer/in:

\section{Prüfungskommission}

Referent/in

Ko-Referent/in:

Datum der mündlichen Prüfung:
Prof. Dr. med. W. Brück

Prof. Dr. med. G. Felmerer

Priv.-Doz. Dr. med. M. Grade

Prof. Dr. med. G. Felmerer

Prof. Dr. med. H. Baraki

14. Dezember 2021 
Hiermit erkläre ich, die Dissertation mit dem Titel

"Der roboterassistierte Lymphknotentransfer mittels vaskularisiertem Omentumlappen in der Therapie des sekundären Lymphödems der Extremitäten" eigenständig angefertigt und keine anderen als die von mir angegebenen Quellen und Hilfsmittel verwendet zu haben.

Göttingen, den 
Die Daten, auf denen die vorliegende Arbeit basiert, wurden teilweise publiziert:

Felmerer G, Behringer D*, Emmerich N, Grade M, Stepniewski A (2021): Donor defects after lymph vessel transplantation and free vascularized lymph node transfer: A comparison and evaluation of complications. World J Transplant 11, 129-137

*geteilte Erstautorenschaft 


\section{Inhaltsverzeichnis}

Abbildungsverzeichnis ................................................................................ III

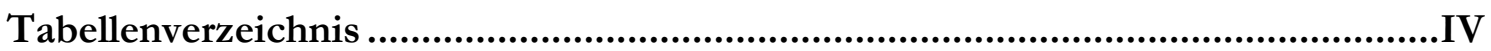

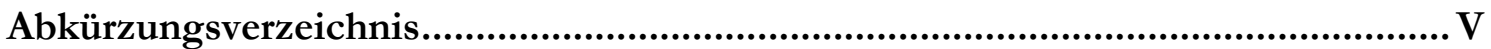

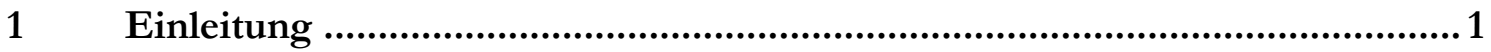

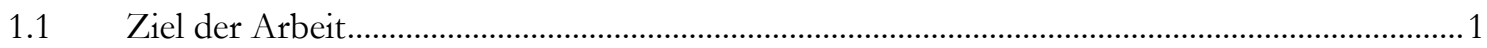

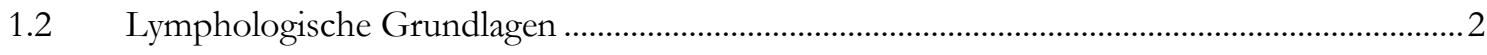

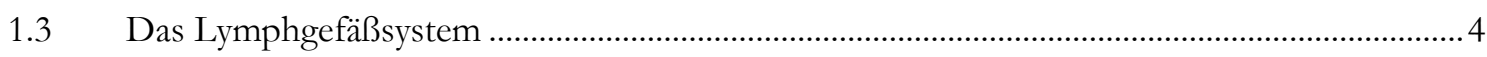

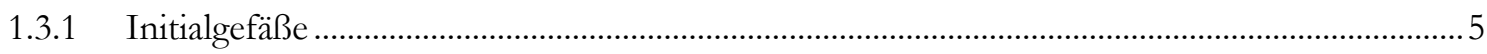

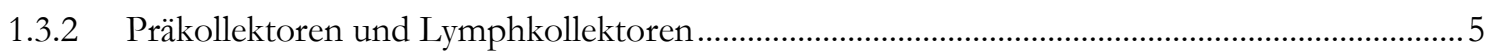

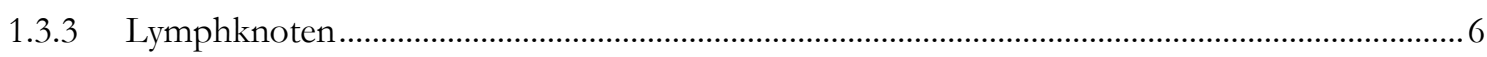

1.3.4 Lymphgänge und Lymphstämme …………………...........................................................

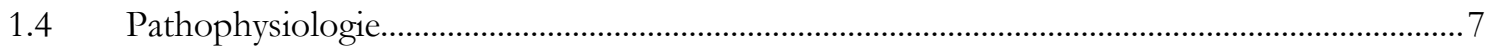

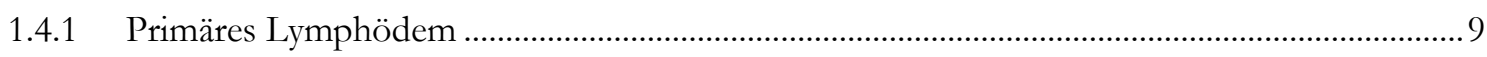

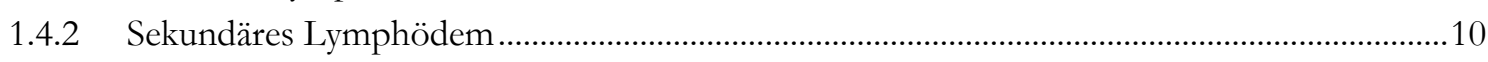

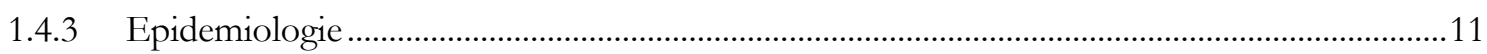

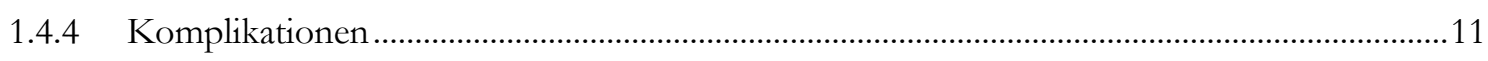

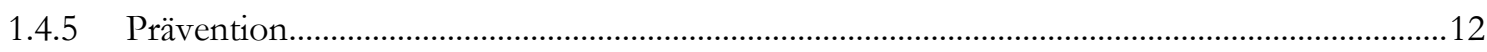

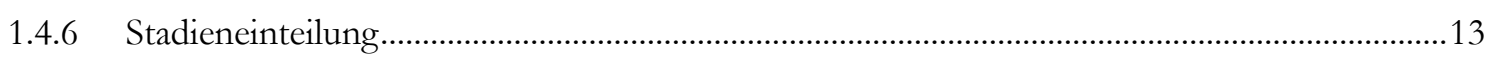

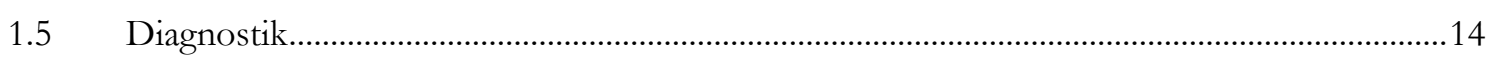

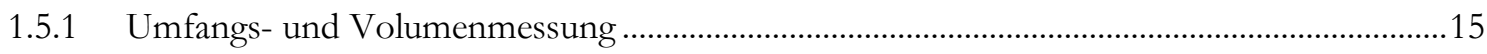

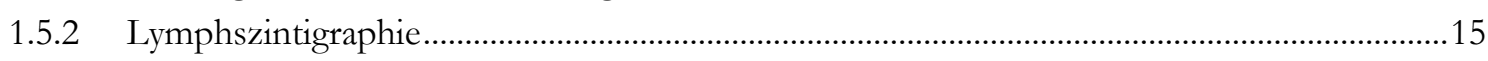

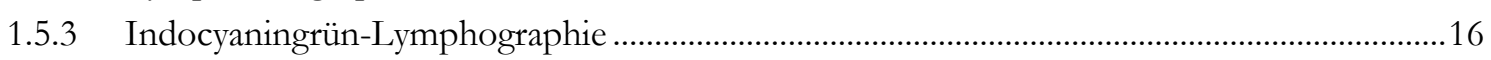

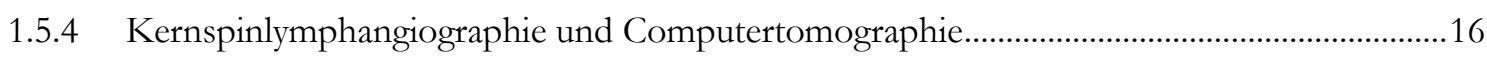

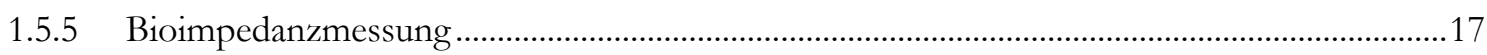

1.5.6 Der prozentuale Wassergehalt der Haut und die Dielektrizitätskonstante ...............................17

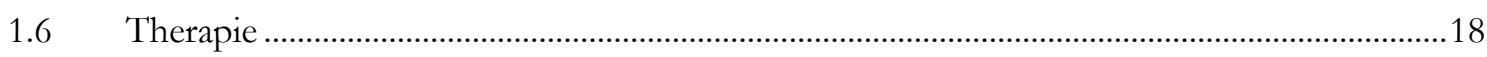

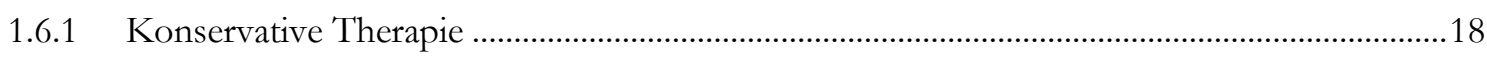

1.6.2 Medikamentöse Therapie .................................................................................................. 19

1.6.3 Chirurgische Therapie....................................................................................................

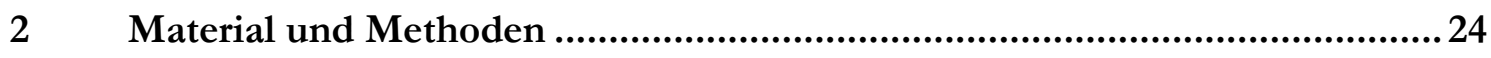

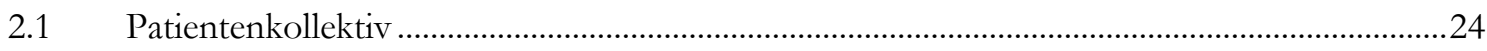

2.1.1 Autologe omentale Lymphknotentransplantation roboterassistiert........................................24

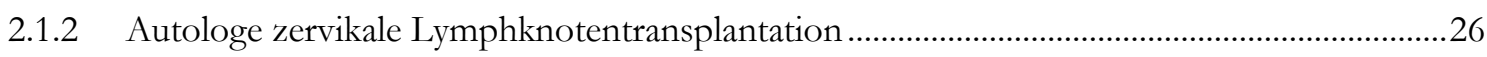

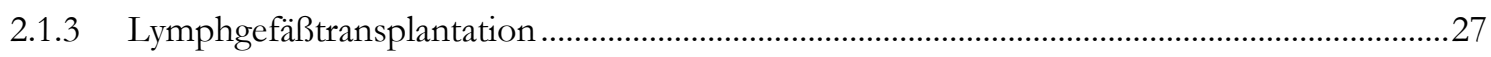

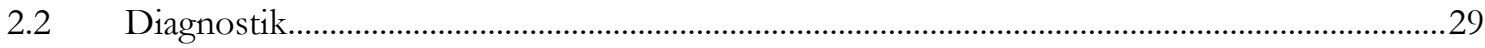




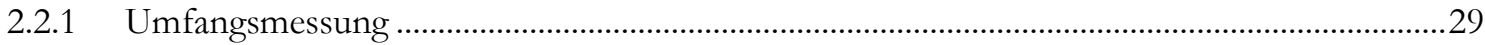

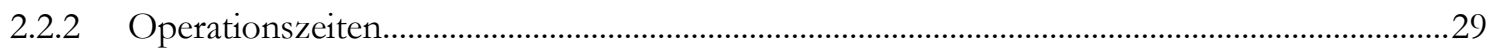

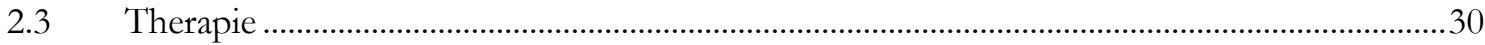

2.3.1 Autologe omentale Lymphknotentransplantation roboterassistiert.......................................31

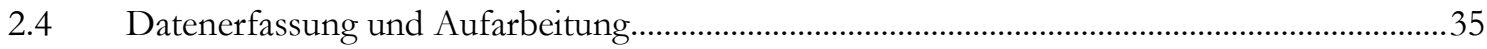

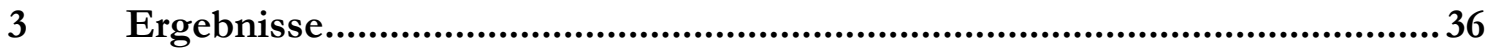

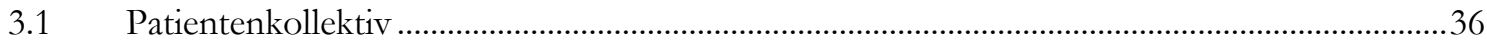

3.1.1 Autologe omentale Lymphknotentransplantation roboterassistiert......................................36

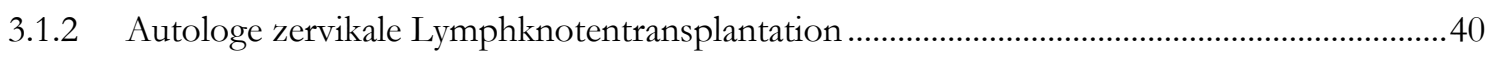

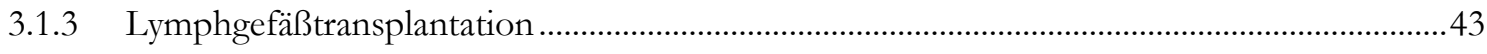

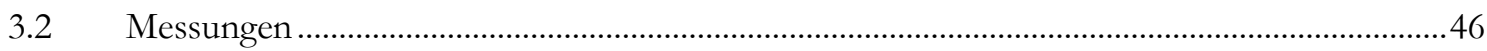

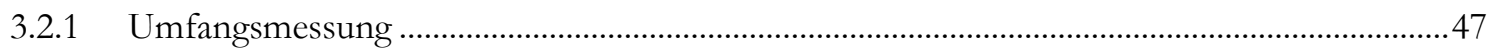

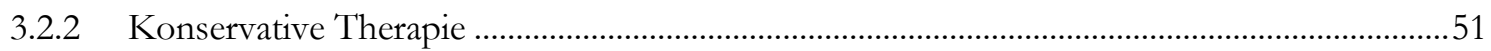

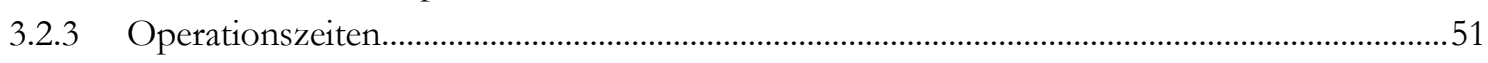

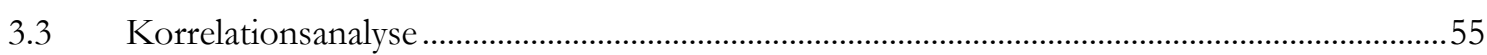

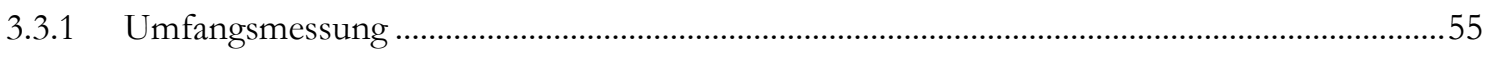

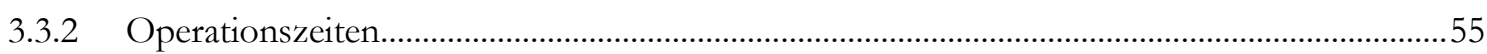

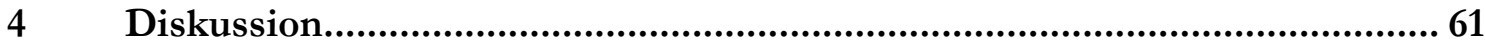

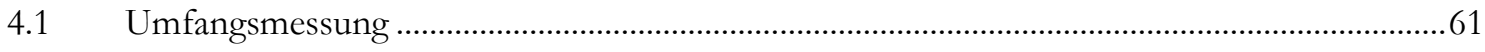

4.2 Mikrochirurgische Verfahren und ihre Hebedefekte.............................................................63

4.2.1 Autologe omentale Lymphknotentransplantation roboterassistiert......................................63

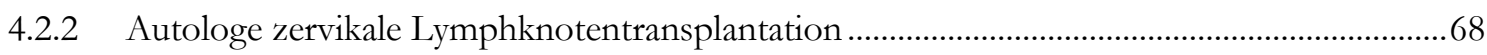

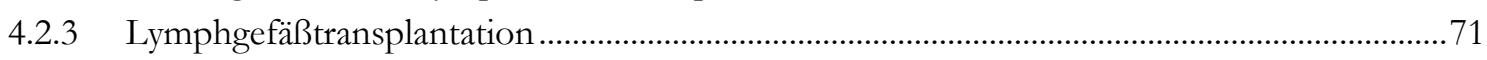

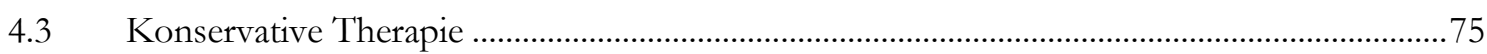

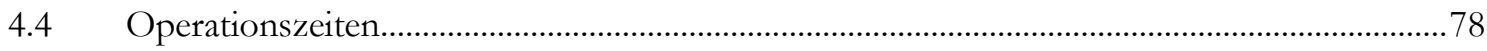

4.4.1 Autologe omentale Lymphknotentransplantation roboterassistiert.......................................79

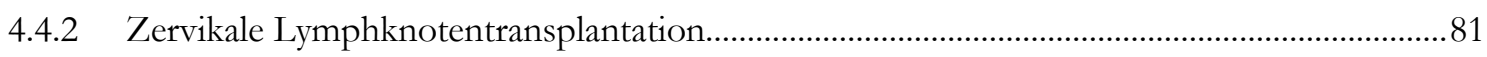

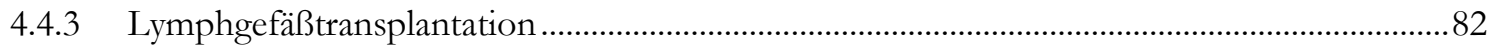

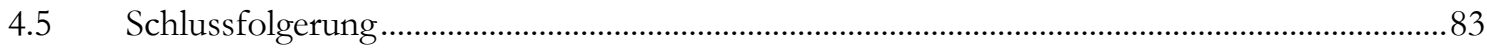

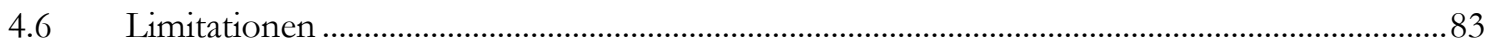

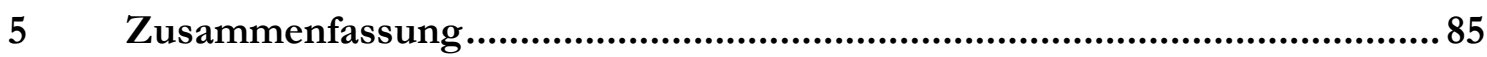

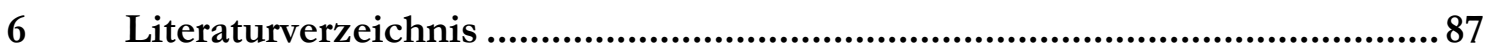




\section{Abbildungsverzeichnis}

Abbildung 1: Grafische Darstellung der Spenderregionen der einzelnen Eingriffe.

Abbildung 2: Übersichtsschema der Lymphchirurgie in der Plastischen Chirurgie in unserer Abteilung.

Abbildung 3: Da-Vinci-Operationsroboter in angedocktem Zustand................................................33

Abbildung 4: Omentaler Lymphknotenlappen nach Bergung aus dem Abdomen. ...........................34

Abbildung 5: Grafische Darstellung der konservativen Therapie des Gesamtkollektives. .................37

Abbildung 6: Grafische Darstellung der konservativen Therapie der Beinlymphödeme....................39

Abbildung 7: Grafische Darstellung der konservativen Therapie der Armlymphödeme...................39

Abbildung 8: Grafische Darstellung der konservativen Therapie des zervikalen Gesamtkollektivs.

Abbildung 9: Grafische Darstellung der konservativen Therapie der zervikalen Armlymphödeme.

Abbildung 10: Grafische Darstellung der konservativen Therapie der zervikalen Beinlymphödeme.

Abbildung 11: Grafische Darstellung der konservativen Therapie bei Lymphgefäßtransplantation.

Abbildung 12: Mittelwerte der Extremitätenumfänge prä- sowie postoperativ in Zentimeter ..........47

Abbildung 13: Mittelwerte der Extremitätenumfänge prä- sowie postoperativ in Zentimeter an der oberen Extremität.

Abbildung 14: Mittelwerte der Extremitätenumfänge prä- sowie postoperativ in Zentimeter an der unteren Extremität.....

Abbildung 15: Box Plots mit Umfangswerten in Zentimetern, Minimum, Maximum, Median und untere und obere Perzentile des Gesamtkollektivs

Abbildung 16: Box Plots mit Umfangswerten in Zentimetern, Minimum, Maximum, Median und untere und obere Perzentile der oberen Extremitäten

Abbildung 17: Box Plots mit Umfangswerten in Zentimetern, Minimum, Maximum, Median und untere und obere Perzentile der unteren Extremitäten

Abbildung 18: Operationszeiten in Minuten; Gesamtoperationszeit und Operationszeit mit dem Da-Vinci-Roboter jeweils mit Minimum, Maximum, Konfidenzintervall, Median und entsprechenden Ausreißern.

Abbildung 19: Operationszeit der zervikalen Lymphknotentransplantation mit Minimum, Maximum, Konfidenzintervall, Median und entsprechenden Ausreißern

Abbildung 20: Operationszeit der Lymphgefäßtransplantationen mit Minimum, Maximum, Konfidenzintervall, Median und entsprechenden Ausreißern.

Abbildung 21: Grafische Darstellung der Operationsminuten des Da-Vinci-Systems mit potenzierte Trendlinie zur Näherung des Kurvenverlaufes

Abbildung 22: Grafische Darstellung der Gesamtoperationszeit mit potenzierte Trendlinie zur Näherung des Kurvenverlaufes

Abbildung 23: Operationszeit in Minuten der durchgeführten zervikalen Lymphknoteneingriffe mit angepasster Trendlinie und entsprechender Funktion

Abbildung 24: Operationszeit in Minuten der durchgeführten Lymphgefäßtransplantationen mit angepasster Trendlinie und entsprechender Funktion..

Abbildung 25: Die einzelnen Operationszeiten der jeweiligen Operationsmethode im zeitlichen Vergleich. 


\section{Tabellenverzeichnis}

Tabelle 1: Stadieneinteilung des Lymphödems nach der ISL...........................................................14

Tabelle 2: Komplikationsübersicht nach zervikaler Lymphknotentransplantation ............................43

Tabelle 3: Komplikationsübersicht nach Lymphgefäßtransplantation .................................................46

Tabelle 4: Mittelwerte \pm 0,95 Konfidenzintervall in Zentimetern präoperativ, nach sechs und nach 12 Monaten für das Gesamtkollektiv sowie die obere und untere Extremität..............49

Tabelle 5: Übersicht der konservativen Therapien im Vergleich (insgesamt sowie aufgeteilt nach entsprechendem Eingriff)

Tabelle 6: Testung auf Normalverteilung des omentalen Patientenkollektivs mittels SPSS .55

Tabelle 7: Korrelationsanalyse der Gesamtoperationszeiten mit der Operationsanzahl für die omentalen Lymphknotentransplantationen..........................................................................56

Tabelle 8: Korrelationsanalyse der Da-Vinci-Operationszeiten mit der Operationsanzahl ................56

Tabelle 9: Korrelationsanalyse für die zervikalen Operationszeiten ....................................................58

Tabelle 10: Korrelationsanalyse für die Lymphgefäßtransplantationen................................................59

Tabelle 11: Berechnete Näherung der erwarteten Operationszeiten ...................................................8 80 


\section{Abkürzungsverzeichnis}

AWMF Arbeitsgemeinschaft der Wissenschaftlichen Medizinischen Fachgesellschaften

FDA Food and Drug Administration

ICGL Indocyaningrün-Lymphographie

ISL International Society of Lymphology

K Filtrationskoeffizient

KI Konfidenzintervall

KPE Komplex Physikalische Entstaungstherapie

LGtx Lymphgefäßtransplantation

LKtx Lymphknotentransplantation

LVA Lymphovenöse Anastomose

pAVK periphere arterielle Verschlusskrankheit

$P_{\text {eff }} \quad$ effektiver Filtrationsdruck

$\mathrm{P}_{\mathrm{i}} \quad$ interstitieller hydrostatischer Druck

$\mathrm{P}_{\mathrm{k}} \quad$ intravasal hydrostatischer Druck

VEGF-C vascular endothelial growth factor C

VEGFR 3 vascular endothelial growth factor receptor 3

WHO World Health Organization

$\pi_{\mathrm{i}} \quad$ interstitieller kolloidosmotischer Druck

$\pi_{\mathrm{k}} \quad$ intravasal kolloidosmotischer Druck

$\sigma \quad$ dimensionsloser Reflexionskoeffizient 


\section{Einleitung}

\subsection{Ziel der Arbeit}

Das sekundäre Lymphödem nach chirurgischen Eingriffen ist eine fortschreitende, sich chronifizierende Erkrankung, welche bis heute nicht komplett heilbar ist. In der Literatur sind über die vergangenen Jahre eine Vielzahl an chirurgischen Therapiemöglichkeiten beschrieben worden (Felmerer et al. 2012; Koshima et al. 2003; Becker 2016; Koshima et al. 2016; Karri et al. 2011; Goldsmith 1974). Bis heute werden die unterschiedlichen Möglichkeiten je nach Operateur, Klinik und Patientenvoraussetzungen divers angewandt. In dieser Arbeit soll auf eine neu, im Schwerpunkt Plastische Chirurgie der Klinik für Unfallchirurgie, Orthopädie und Plastische Chirurgie, etablierte Methode der Lymphknotentransplantation aus dem Omentum (Goldsmith und los Santos 1967), in roboterassistierter Technik, eingegangen werden. Als Vergleichsmaterial soll die autologe supraklavikuläre Lymphknotentransplantation (LKtx) (Becker 2016) sowie die Lymphgefäßtransplantation (LGtx) nach Baumeister (Baumeister 1983; Baumeister et al. 1986) verwendet werden.

Die beiden letztgenannten Therapieoptionen werden seit Etablierung der Mikrochirurgie international sowie auch seit längerer Zeit im Schwerpunktbereich der Plastischen Chirurgie an der Universitätsmedizin in Göttingen angewandt und zeigten hier bereits vielversprechende Ergebnisse (Batista et al. 2017; Cheng et al. 2013; Klingelhoefer et al. 2019; Stepniewski et al. 2019). Die erstgenannte operative Methode stellt, in ihrer bei uns durchgeführten Form, eine Neuheit dar und kombiniert die Vorteile eines minimal invasiven Eingriffes durch den Da-Vinci-Operationsroboter mit den bereits bekannten Vorteilen der Lymphknotentransplantation.

Da es sich um einen Eingriff mit Eröffnung des Bauchraumes handelt, sollten in dieser Arbeit eventuelle abdominelle Komplikationen herausgearbeitet werden. Diese sollen mit der Hebedefektmorbidität der anderen beiden Eingriffe verglichen werden. Unter Berücksichtigung der Operationszeit, und Veränderung dieser im Verlauf, soll dargestellt werden, ob der Einsatz des Da-Vinci-Operationsroboters im klinischen Alltag praktikabel und sinnvoll ist. 
Die Datenerhebung des omentalen Patientenkollektivs soll jeweils präoperativ bei Vorstellung in der Sprechstunde, perioperativ sowie drei Wochen, sechs Monate und zwölf Monate nach operativer Versorgung durchgeführt werden.

Ziel dieser Arbeit ist es somit die Rolle, Praktikabilität, Möglichkeiten und Komplikationen des roboterassistierten Lymphknotentransfers in der Behandlung des sekundären Extremitätenlymphödems im Verglich zu bereits bekannten Eingriffen zu beleuchten.

\subsection{Lymphologische Grundlagen}

Das lymphatische System besteht aus einem nach zentripetal gerichteten Gefäßsystem, welches parallel zum venösen Gefäßsystem verläuft und für die Homöostase des menschlichen Körpers wichtig ist. Hierbei übernimmt es Aufgaben, die in der Aufrechterhaltung des Elektrolyt- und Wasserhaushaltes sowie der immunologischen Abwehrreaktion eine wesentliche Rolle spielen (Kubik 2002). Die Lymphflüssigkeit in den Lymphgefäßen dient dazu, die in der Peripherie anfallende interstitielle Flüssigkeit sowie die darin vorhandenen Proteine, Immunzellen und Stoffwechselprodukte in das Blutgefäßsystem des Körpers zurückzuführen (Lüllmann-Rauch 2015; Smith et al. 1970; Borgstrom und Laurell 1953). Zusätzlich zu den bereits genannten Inhaltsstoffen sind in der Lymphflüssigkeit Gerinnungsfaktoren und Fibrinogen enthalten, diese können zu einer plasmatischen Gerinnung führen (Stutman et al. 1965; Lippi et al. 2012). Die Zusammensetzung der Lymphflüssigkeit ist in den ganz distal gelegenen Initialgefäßen quasi äquivalent zur interstitiellen Flüssigkeit des Körpers (Lüllmann-Rauch 2015). Je nach Körperregion gibt es jedoch auch deutliche Unterschiede. Wichtige Faktoren sind hierbei die Blutbestandteile der entsprechenden Körperregion, die Basalmembran und die Konfiguration des Endothels der Blutkapillaren (kontinuierlich, diskontinuierlich, fenestriert). Als Beispiele sind hier die proteinreiche Lymphflüssigkeit der Leber (Lüllmann-Rauch 2015) oder die fettreiche und chylomikronhaltige Flüssigkeit der intestinalen Drainageregion zu nennen (Dixon et al. 2009; Tso und Balint 1986; Azzali 1982). Die Lymphe stellt als wichtiger Bestandteil des Immunsystems, zusätzlich zu oben genannten Funktionen, ein Trägermedium für antigenpräsentierende Zellen, Antigene und Leukozyten dar. Durch das nach zentripetal gerichtete System erfolgt der Transport zu den Lymphknoten, in denen eine Antigenpräsentation an die Lymphozyten erfolgen kann (Sixt et al. 2005; Roozendaal et al. 2008).

Das tägliche Lymphflüssigkeitsvolumen ist stark vom Flüssigkeitshaushalt des Körpers abhängig und beträgt durchschnittlich zwischen zwei und drei Liter. Bei entsprechend 
hohem Flüssigkeitshaushalt kann es aber auch auf das 20- bis 100-fache ansteigen (Brandes und Busse 2010). Diese Anpassungsfähigkeit ist zur Aufrechterhaltung der Hämostase der Flüssigkeitskompartimente des Körpers notwendig. Die Lymphflüssigkeit entsteht während der Mikrozirkulation in den Blutkapillaren durch Filtration. Die physikalisch wirkenden Größen sind hierbei der intravasale hydrostatische Druck $\left(\mathrm{P}_{\mathrm{k}}\right)$, der kolloidosmotische Druck $\left(\pi_{\mathrm{k}}\right)$, sowie der hydrostatische $\left(\mathrm{P}_{\mathrm{i}}\right)$ und kolloidosmotische Druck $\left(\pi_{\mathrm{i}}\right)$ in der interstitiellen Flüssigkeit. Je nach Höhe der einzelnen Größen, beziehungsweise deren Differenz, kommt es zu unterschiedlich starker Filtration und Reabsorption in den Blutkapillaren. Die somit wirkende Kraft wird nach E. H. Starling (Starling 1896) als effektiver Filtrationsdruck ( $\mathrm{P}_{\text {eff }}$ ) aus den Kapillaren in das Interstitium beschrieben.

$$
P_{\text {eff }}=\left(P_{k}-P_{i}\right)-\sigma\left(\pi_{k}-\pi_{i}\right)=\Delta P-\sigma \Delta \pi
$$

In dieser Formel stellt $\sigma$ einen dimensionslosen Reflexionskoeffizienten dar, der das Maß für die Durchlässigkeit der Kapillarwand angibt. Wenn $\sigma=0$ annimmt, wird Wasser und alle in ihm gelösten Substanzen und Teilchen ausnahmslos filtriert. Die Kapillarwand stellt somit keine Durchlässigkeitsbarriere dar. Für $\sigma=1$ kann ausschließlich Wasser durch die Kapillarwand dringen. Es handelt sich um eine ideale semipermeable Membran (Staverman 1951). Bei Entzündungsreaktionen kommt es zu einer Verringerung von $\sigma$, was zur Veränderung der Membranpermeabilität führt. Als Resultat der Veränderung können vermehrt Eiweißmoleküle ins Interstitium gelangen (Földi und Földi 2002b).

Zusätzlich zum effektiven Filtrationsdruck stellen auch die Kapillarwandfläche des entsprechenden Gefäßes und die hydraulische Leitfähigkeit einen entscheidenden Faktor dar. Als Produkt ergeben sie den Filtrationskoeffizienten K. Aus den bereits aus der vorherigen Formel bekannten Werten sowie K lässt sich $Q_{f}$ berechnen. Er ist definiert als das über die Gefäßwand pro Zeiteinheit filtrierte Flüssigkeitsvolumen (Ehmke 2014).

$$
Q_{f}=K(\Delta P-\sigma \Delta \pi)
$$

Die Kapillarpermeabilität, Proteinkonzentration im Plasma sowie der arterielle und venöse Blutdruck stellen jeweils nach Stoffwechsellage variable Einflussfaktoren auf $Q_{f}$ dar (Wiig und Swartz 2012).

Bei Betrachtung der Mikrozirkulation eines Gefäßmodells ist $\Delta P$ für den arteriellen Bereich immer größer als $\Delta \pi$. Dadurch kommt es zu einer Filtration ins Interstitium in der betrachteten Region. Umso weiter sich die entsprechende Region vom arteriellen Bereich entfernt, desto kleiner wird auch $\Delta P$. Sobald der $\Delta P$ Wert kleiner als der $\Delta \pi$ Wert wird, 
resultiert eine Reabsorption der Flüssigkeit in das Gefäß (Ehmke 2014). Von den täglich ca. 20 Litern produziertem Filtrat erfolgt eine Reabsorption von ca. 18 Litern, also 80\%, in den postkapillären Venolen. Die restlichen zwei Liter werden über das Interstitium und durch die Lymphgefäße abgeleitet (Brandes und Busse 2010).

Ob Moleküle aus der interstitiellen Flüssigkeit in die venösen Gefäße reabsorbiert werden oder ob sie über das Lymphgefäßsystem dem venösen System zugeführt werden, erfolgt größenabhängig. Genaue Grenzwerte sind hier nicht bekannt (Swartz 2001; Porter 1997; Swartz et al. 1996; Strand und Persson 1979).

Der Weitertransport der interstitiellen Flüssigkeit in die Lymphgefäße erfolgt durch den Druckgradienten zwischen Interstitium und Lymphkapillare (Swartz 2001). Durch die rhythmischen Kontraktionen der Tunica media der glatten Muskulatur wird ein negativ hydrostatischer Druck (Guyton 1963; Guyton et al. 1971) erzeugt, welcher diesen Druckgradienten aufrechterhält (Olszewski und Engeset 1980). Unterstützt wird der Druckgradient durch die Gefäßklappen, welche einen Rückfluss verhindern sowie die Kontraktion der angrenzenden Arterien, die Muskelpumpe der Extremitäten (Olszewski und Engeset 1980) und die sich verändernden Druckgradienten bei In- und Exspiration (Schmid-Schönbein 1990; Schad et al. 1978). Jeder einzelne Mechanismus trägt dazu bei, den zentripetalen Fluss der Lymphe zu gewährleisten und den Druckgradienten in den Lymphkapillaren permanent aufrecht zu erhalten. Daraus resultiert ein bedarfsgerechter Abtransport der zusätzlichen interstitiellen Flüssigkeit über die Lymphgefäße zurück in den Blutkreislauf (Schmid-Schönbein 1990).

\subsection{Das Lymphgefäßsystem}

Das Lymphgefäßsystem unterteilt sich von distal nach proximal in Lymphkapillaren, Präkollektoren, Lymphkollektoren, Lymphstämme und Lymphgänge. Zusammenfassend spricht man bei den Lymphkapillaren und Präkollektoren auch von Initialgefäßen. Zwischen die einzelnen Lymphkollektoren sind Lymphknoten geschaltet, die Filterfunktionen und diverse immunologische Aufgaben übernehmen (Kubik 2002).

Das Drainagesystem wird, je nach Lokalisation, in drei Kompartimente unterteilt. Man unterscheidet ein oberflächliches System, welches die Lymphe der Haut und der Subkutis im Bereich der Extremitäten abfließen lässt, von dem tiefen System, welches Gelenke, Nerven, Sehnenscheiden und Muskeln lymphatisch drainiert. Die Lymphgefäße des tiefen Systems laufen in einer Gefäßscheide mit den tief gelegenen großen Blutgefäßen des Körpers. Das epifaszial liegende oberflächliche Gefäßsystem und das subfasziale tiefe 
Gefäßsystem sind durch Perforansgefäße miteinander verbunden. Das organspezifische System stellt das dritte Kompartiment der Drainagesysteme dar. Es ist sehr stark an die individuellen Organe angepasst und funktioniert daher unterschiedlich, je nach Bedürfnissen und Stoffwechselfunktionen (Kubik 2002).

\subsubsection{Initialgefäße}

Die Lymphkapillaren bilden ein feinmaschiges Gefäßnetz im Bindegewebe des Interstitiums. Über ihre zwischen den Fibrozyten des Bindegewebes endende Fortsätze, können sie die interstitielle Flüssigkeit aufnehmen (Leak 1976; Hauck 1972; Hauck und Castenholz 1992). Ihr Durchmesser unterscheidet sich mit 10-60 $\mu \mathrm{m}$ deutlich von dem der ca. $6 \mu \mathrm{m}$ großen Blutkapillaren (Swartz 2001; Schmid-Schönbein 1990). Durch ihren übereinanderliegenden dachziegelartigen Aufbau aus Endothelzellen entsteht eine klappenähnliche Formation, welche die Flüssigkeitsaufnahme ermöglicht und einen Rückfluss verhindert (Trzewik et al. 2001; Leak 1976).

\subsubsection{Präkollektoren und Lymphkollektoren}

Die zwischen 35 und $150 \mu \mathrm{m}$ großen Präkollektoren verbinden die Lymphkapillaren mit den pränodal liegenden Lymphkollektoren. Ihr Endothel besitzt kleine Öffnungen, wodurch sie sowohl zur Weiterleitung der Lymphflüssigkeit als auch zur Absorption der interstitiellen Flüssigkeit befähigt sind (Sacchi et al. 1997).

In ihrem Aufbau ähneln die Lymphkollektoren kleinen Venen. So besitzen sie beispielsweise eine Tunica media, die eine Zweischichtung bestehend aus longitudinaler und zirkulärer Muskelschicht besitzt (Hasselhof et al. 2016). Weiter sind in ihrer Wand regelmäßig angeordnete Klappen aus Bindegewebe zu finden (Schmid-Schönbein 1990). Der Abschnitt einer Klappe zur nächsten wird als Lymphangion bezeichnet. Bei aktiver Muskelkontraktion kommt es dann zum gerichteten Transport unidirektional entlang des geringsten Wiederstandes von Lymphangion zu Lymphangion (Gashev 2008; SchmidSchönbein 1990). Zwar spielen auch passive Mechanismen beim Transport der Lymphflüssigkeit eine Rolle, die wichtigste Komponente ist jedoch die Fähigkeit der aktiven Kontraktion (Hasselhof et al. 2016; Olszewski und Engeset 1980).

Durch Anastomosen und Kollateralgefäße zwischen den einzelnen Hauptkollektoren ergibt sich ein variabler Gefäßverlauf, welcher auch bei Verschluss einzelner Gefäße eine ausreichende Drainage sicherstellen kann (Kubik 2002). 


\subsubsection{Lymphknoten}

Die Lymphknoten sind zahlreiche, dem Lymphsystem zugeordnete, Filterstationen des menschlichen Körpers. Die größte Anzahl ist in der Achselhöhle, dem thorakalen Mediastinum und dem Gastrointestinaltrakt vorhanden. Die einzelnen Lymphknoten liegen in Gruppen oder Ketten zwischen den Lymphkollektoren. Hierbei sind primäre Lymphknoten direkt einem bestimmten Areal zugeordnet während, sekundäre, auch Sammellymphknoten genannte Lymphknoten, den primären nachgeschaltet sind. Das Areal, welches ein Lymphknoten drainiert, nennt sich tributäres Gebiet (Lüllmann-Rauch 2015; Földi und Strössenreuther 2004; Kubik 2002).

Insgesamt sind im menschlichen Körper zwischen 600 bis 700 Lymphknoten vorhanden, mit erheblichen geschlechterspezifischen Unterschieden bezüglich ihrer Anzahl und Größe (Kubik 2002; Willard-Mack 2006). Makroskopisch können sie, je nach anatomischer Region, rund, oval, spindel- oder nierenförmig erscheinen und variieren mit einer Größe von 0,2 bis 3 Zentimetern. Die Lymphe wird über afferente Lymphgefäße zu den Lymphknoten hin und über efferente Lymphgefäße von ihnen wegtransportiert. Bei den afferenten Lymphgefäßen kann es sich um periphere Lymphkollektoren oder um internodale Verbindungsäste handeln (Lüllmann-Rauch 2015; Pabst 2004).

Mikroskopisch bestehen die Lymphknoten aus einer Kapsel mit dicht gepackten Kollagenfasern, wenigen elastischen Fasern und einigen glatten Muskelzellen. Von der Kapsel ziehen Bindegewebstrabekel in den Lymphknoten und unterteilten diesen unvollständig in einzelne Kompartimente. Das Grundgerüst des Lymphknotens besteht aus retikulärem Bindegewebe. Von außen nach innen unterteilt man den Lymphknoten in Cortex, Paracortex und Medulla (Welsch und Deller 2010; Willard-Mack 2006; Pabst 2004). In der Cortex befinden sich primäre und sekundäre Follikel, die größtenteils aus BLymphozyten aufgebaut sind. In der Paracortex sind primär T-Lymphozyten vorhanden, welche in ihrem Aufbau deutlich weniger dicht gepackt sind (Lüllmann-Rauch 2015; Weiss 1973). Die in der Paracortex lokalisierten hochendothelialen Venolen gelten als wichtiger Ort der Lymphozytenrezirkulation und sind an den immunologischen Reaktionen des Körpers beteiligt (Pabst 2004). 


\subsubsection{Lymphgänge und Lymphstämme}

Die Lymphe der unteren Extremität wird über die Cisterna chyli und den Ductus thoracicus, gemeinsam mit der Lymphe der linken oberen Extremität, in den linken Venenwinkel abgeleitet. Die Ableitung der rechten oberen Extremität erfolgt direkt in den rechten Venenwinkel. Gespeist werden die Lymphstämme durch die den Lymphknoten verlassenden, efferenten Lymphgefäße. Zusätzlich zur Lymphe der unteren Extremität und des Magen-Darm-Traktes nimmt der Ductus thoracicus auch die Lymphe aus den tiefen Schichten des Rückens, den paravertebralen Pleuraabschnitten und dem unteren Mediastinum auf. Eine Vielzahl von Normvarianten sind hierbei beschrieben und können regelhaft auftreten (Johnson et al. 2016; Kubik 2002; Boggon und Palfrey 1973).

Der Ductus thoracicus stellt den größten Lymphstamm dar und beginnt in Form der Cisterna chyli zwischen dem zweiten Lendenwirbelkörper und dem zehnten Brustwirbelkörper. Sie bekommt Zulauf von den Trunci lumbales sinister et dexter sowie dem Truncus intestinalis und steigt dann im hinteren Mediastinum bis zur Basis des Halses auf, wo sie normalerweise in den linke Venenwinkel einmündet (Drake et al. 2005; Kiyonaga et al. 2012). Topographisch unterteilt man den Ductus thoracicus in einen abdominalen, thorakalen und zervikalen Teil. Der abdominale Teil ist retroperitoneal hinter oder rechts neben der Aorta gelegen. Über den Hiatus aorticus steigt der Ductus thoracicus in den Thorax auf und liegt dann zwischen Aorta und Vena asygos hinter der Speiseröhre (Drake et al. 2005). Auf Höhe des vierten bis fünften Brustwirbels verläuft er in bogenförmiger Richtung und kommt zwischen der Speiseröhre und der linken $A$. subclavia zu liegen. Hier nimmt er die Lymphe aus dem Truncus bronchomediastinalis sinister, subclavius sinister und jugularis sinister auf und mündet dann in den linken Venenwinkel (Smith et al. 2013; Kinnaert 1973). Die Länge des Ductus thoracicus variiert zwischen 36 und 45 Zentimetern. Am Eintrittspunkt im linken Venenwinkel finden sich spezielle Gefäßklappen und eine ampulläre Aufweitung, die zusätzlich zur schrägen Eintrittsrichtung einen Reflux von venösem Blut in den Ductus verhindert (Kubik 2002; Pflug und Calnan 1968).

\subsection{Pathophysiologie}

Laut aktueller AWMF-Leitlinie (Arbeitsgemeinschaft der Wissenschaftlichen Medizinischen Fachgesellschaften) der Gesellschaft Deutschsprachiger Lymphologen handelt es sich beim Lymphödem im unbehandelten Zustand um „eine chronische, entzündliche Erkrankung des Interstitiums als Folge einer primären (anlagebedingten) oder sekundären (erworbenen) Schädigung des Lymphdrainagesystems [...]. Ein insuffizient gewordenes 
Lymphdrainagesystem führt zur konsekutiven Vermehrung und Veränderung der interstitiellen Gewebsflüssigkeit. Im weiteren Verlauf ist die Erkrankung durch eine Alteration von Geweben gekennzeichnet, unabhängig von ihrer anatomischen Lokalisation“ (Leitlinie Lymphödem 2017).

In der Pathophysiologie des Lymphödems wird grundsätzlich eine dynamische Insuffizienz (Hochvolumeninsuffizienz) von einer mechanischen Insuffizienz (Niedrigvolumeninsuffizienz) unterschieden. Prinzipiell ist aber auch eine Kombination aus beiden Insuffizienzformen möglich.

Zur dynamischen Insuffizienz kommt es durch ein permanentes Überschreiten der Transportkapazität aufgrund einer pathologisch erhöhten lymphatischen Last. Bei der mechanischen Insuffizienz kommt es zum Überschreiten einer pathologisch erniedrigten Transportkapazität bei normaler lymphatischer Last.

Bei der Kombination beider Formen ist eine pathologisch erhöhte lymphatische Last sowie auch eine pathologisch erniedrigte Transportkapazität vorhanden. Man spricht dann auch von Sicherheitsventilinsuffizienz (International Society of Lymphology 2013; Földi und Földi 2002a; Földi et al 1987).

Unter lymphatischer Transportkapazität wird die Fähigkeit ein entsprechendes Lymphvolumen aus einem Gewebeanteil zu drainieren verstanden. Im Normalfall beträgt die lymphatische Transportkapazität nur ca. 10\% der tatsächlich möglichen Gesamtkapazität. Sie kann jedoch deutlich gesteigert werden und somit Schwankungen des Lymphvolumens kompensieren. Diese Möglichkeit wird auch als Sicherheitsventilfunktion und funktionelle Reserve bezeichnet (Földi et al 1987; Földi und Földi 2002a).

Der Ursprung des Lymphödems kann sehr vielfältig sein und bis heute sind noch nicht alle pathophysiologischen Vorgänge bekannt. Durch eine Störung im Abfluss der Lymphflüssigkeit kommt es zum Rückstau bis in die Peripherie. Zu Beginn können die noch intakten Lymphgefäße diesen Rückstau, durch ihre Sicherheitsventilfunktion und Ausschöpfung ihrer funktionellen Reserve, kompensieren. Bei anhaltender Belastung, durch ein erhöhtes interstitielles Volumen, kommt es jedoch zur Ermüdung der glatten Muskulatur in der Lymphgefäßwand (Casley-Smith et al. 1980; Lewis und Wald 1984). Zusätzlich folgt aus dem erhöhten intravasalen Druck eine Überdehnung der Lymphgefäßklappen, wodurch diese sich nicht mehr vollständig schließen (Mortimer 1998; Olszewski 2008). Dies hat einen Rückfluss der Lymphflüssigkeit, gegen ihre eigentliche Flussrichtung nach zentripetal, zur Folge (Olszewski 2008). Durch die immer weiter 
ansteigende Lymphflüssigkeit im Interstitium kommt es hier zur Erhöhung des osmotischen Druckes, welcher im Verlauf die einzelnen Bestandteil des Bindegewebes schädigt und pathologisch verändert (Swartz 2001; Kaiserling 2002). Zusätzlich kann es, aufgrund der proteinreichen Flüssigkeit, zur Aktivierung von proinflammatorischen Mediatoren und proteolytischen Enzymen kommen. Es wird ein chronisch progredienter Prozess mit Einwanderung von Immunzellen gestartet. Im Anschluss kommt es durch Fibroblasten, Adipozyten und Makrophagen zu Fibrosklerose und Lipidablagerungen mit Lipidose in den betroffenen Regionen (Földi und Földi 2002a; Kaiserling 2002; Swartz 2001; Casley-Smith und Gaffney 1981; Casley-Smith et al. 1980).

\subsubsection{Primäres Lymphödem}

Primäre Lymphödeme sind Lymphödeme unbekannter oder kongenitaler Ursache (Schook et al. 2011). Auch bei einigen vererbbaren Chromosomendefekten treten sie gehäuft auf (Bellini et al. 2009; Ferrell et al. 1998). Unter den Dysplasien der Lymphgefäße und Lymphknoten werden die Hyperplasie, Hypoplasie und Aplasie zusammengefasst (Liu et al. 2012; Kinmonth und Wolfe 1980).

Eine Aplasie beschreibt im Allgemeinen die Anlage, jedoch fehlende erfolgreiche Entwicklung des entsprechenden Organs oder Gewebes (Pschyrembel 2017). Lediglich Aplasien von Lymphkapillaren und Lymphknoten sind möglich, da eine komplette Aplasie der Lymphgefäße nicht mit dem Leben vereinbar ist (Földi und Földi 2002a).

Bei der Hypoplasie der Lymphgefäße kommt es zu einer verringerten Anzahl sowie verringertem Durchmesser der subfaszial liegenden Lymphkollektoren (Liu et al. 2012; Kinmonth und Wolfe 1980). Hypoplastisch veränderte Lymphgefäße sind besonders häufig beim Ullrich-Turner-Syndrom sowie dem Noonan-Syndrom zu sehen (Alvin et al. 1967; Lanning et al. 1978).

Bei einer Hyperplasie sind vor allem Sammelgefäße und die initialen Lymphgefäße betroffen. Durch Vergrößerung und Zellzunahme kommt es zu Ektasien, was im Verlauf häufig in einer Klappeninsuffizienz resultiert (Liu et al. 2012). Beim autosomal-dominant vererbten hereditären Lymphödem Typ I (Nonne-Milroy-Meige-Syndrom) kommen hyperplastische Lymphgefäße besonders häufig vor. Dies führt neben Minderwuchs, Akromikrie, Adipositas und Hypogonadismus zu Lymphödemen der Extremitäten (Brice et al. 2005; Bollinger et al. 1983).

Eine weitere Unterteilung der primären Lymphödeme ist nach Zeitpunkt der Erstmanifestation möglich. Hierbei wird das Lymphoedema praecox, welches sich postnatal 
vor dem 35. Labensjahr manifestiert, von dem Lymphoedema tardum, welches erst nach dem 35. Lebensjahr auftritt unterschieden (Lewis und Wald 1984).

\subsubsection{Sekundäres Lymphödem}

Beim sekundäre Lymphödem handelt es sich um eine erworbene Erkrankung, die sich daher in jedem Lebensalter manifestieren kann. Bis heute sind viele verschiedene Ursachen bekannt. Im Anschluss wird eine Auswahl an häufigen und wichtigen Ursachen, ohne Anspruch auf Vollständigkeit, beschrieben.

Die laut WHO (World Health Organisation) weltweit häufigste Ursache der sekundären Lymphödeme stellt die Filariose dar. Sie fasst mehrere, durch Stechmücken übertragene, parasitäre Erkrankungen von Fadenwürmer zusammen, welche sich im Lymphgefäßsystem ansiedeln und hier zu entzündlichen Prozessen führen. Durch die wiederkehrenden inflammatorischen Ereignisse kommt es zur Veränderung des Gewebes mit Ektasien und bei vollständiger Ausprägung zur Elephantiasis (Dreyer et al. 2000; World Health Organisation 1992).

Im Gegensatz zu den weltweiten Zahlen macht das iatrogen verursachte sekundäre Lymphöden die häufigste Ursache in den Industrienationen aus. Im Regelfall kommt es hier, nach operativer Versorgung einer Tumorerkrankung mit Lymphonodektomie und postoperativer Radiatio, zur Verletzung und Zerstörung der Lymphbahnen im entsprechenden Bereich (Szuba und Rockson 1998). In weit geringerer Anzahl sind sekundäre Lymphödeme nach Varizenoperation (Hara et al. 2016; Pittaluga und Chastanet 2012), aortokoronarem Venenbypass (Yu et al. 2017), postischämisch bei einer pAVK (periphere arterielle Verschlusskrankheit) oder Spaltung eines Kompartment-Syndroms (Perry und Manoli 2012) beschrieben.

Auch im Rahmen von anderen Primärerkrankungen, wie bei der chronisch venösen Insuffizienz (Eberhardt und Raffetto 2014), der rheumatoiden Arthritis sowie als Folge von Erysipelen, können sekundäre Lymphödeme im Verlauf auftreten (Minari et al. 1994; Inghammar et al. 2014). Die Lymphangiosis carcinomatosa kann, durch invasives Tumorwachstum mit Befall des Lymphgefäßssystem und folglicher Abflusshinderung, zu einem sekundärem Lymphödem führen (Damstra et al. 2010). In der Literatur sind zusätzlich noch Lymphödeme nach mutwilliger Abschnürung von Extremitäten (Rabe 1999; Fátima Guerreiro Godoy und Pereira De Godoy 2015) und Abschnürung durch Haare bereits im Mutterleib, im Sinne eines Tourniquet-Syndroms, beschrieben (Bean et al. 2015). 


\subsubsection{Epidemiologie}

Die vorhandenen Daten zur Epidemiologie des Lymphödems sind häufig nur grob geschätzt und unterliegen, auch aufgrund der vielen nicht erfassten Fällen des sekundären Lymphödems, einer hohen Dunkelziffer. Weiter kommt hinzu, dass sich die Ursachen für Lymphödeme je nach Region und Entwicklungsstand des entsprechenden Staates deutlich unterscheiden (Leitlinie Lymphödem 2017).

Geschätzte Zahlen gehen von einer Inzidenz des primären Lymphödems von ca. 1:6.000 bei Geburt aus (Leitlinie Lymphödem 2017; Dale 1985). Die Gesamtanzahl aller erfassten Fälle soll für die unter 20-Jährigen bei ca. 1:87.000 Betroffenen liegen (Leitlinie Lymphödem 2017; Smeltzer et al. 1985). Die Inzidenz des sekundären Lymphödems wird zwischen 0,13-2 \% in den Industrieländern geschätzt (Leitlinie Lymphödem 2017).

Die Infektionen durch Fadenwürmern stellt weltweit die häufigste Ursache des Lymphödems dar. Schätzungsweise 120 Millionen Menschen leiden laut WHO unter der parasitären Filariose. In den westlichen Industrieländern ist dagegen die chirurgische Therapie von Tumorerkrankungen die häufigste Ursache für die Entstehung sekundärer Lymphödeme. Konkrete Zahlen variieren hier sehr stark (Leitlinie Lymphödem 2017).

\subsubsection{Komplikationen}

Erysipele sind die häufigsten Komplikationen des Lymphödems. Hierbei kommt es zu einer akut bakteriellen Infektion der Haut, die sich dann subepidermal entlang der Lymphgefäße und im Interstitium ausbreitet. Die Eintrittspforte stellen meist kleinste Hautdefekt oder Hautrisse, im Bereich der Zehenzwischenräume, dar (Dupuy et al. 1999). Durch das vorgeschädigten Lymphgefäßsystem sind die entsprechenden Regionen sehr vulnerable und kleinste Erregeranzahlen sind für das Ausbrechen einer Infektion ausreichend (Carlson 2014). Die bakteriellen Erreger sind meist Strepptococcus pyogenes, also $\beta$ hämolysierende Streptokokken der Lancefield-Gruppe A. Durch die stattfindende Entzündungsreaktion kommt es zur verstärkten Belastung des Lymphgefäßssystem und im Verlauf zur Sicherheitsventilinsuffizienz. Bereits erstmalige Entzündungen können somit ein Lymphödem verursachen. Bei schon bestehenden Lymphödemen können Erysipele den klinischen Befund deutlich verschlechtern (Chlebicki und Oh 2014; Földi et al. 2002; Bollinger 1993).

Neben Aufklärung und präventiven Maßnahmen ist eine kalkulierte Antibiotikatherapie, mit Penicillin als Mittel der ersten Wahl, essentiell in der Behandlung des Erysipels. Eine Antibiotikaprophylaxe sollte jedoch immer unter Abwägung der Risiken durchgeführt 
werden und aufgrund von Resistenzentwicklung nur Hochrisikogruppen vorbehalten sein (Chlebicki und Oh 2014).

Eine weitere Komplikation ist die Papillomatosis cutis lymphostatica. Hierbei handelt es sich um eine, bei lang bestehenden Lymphödemen, auftretende Hyperkeratosen der Haut (Wohlrab et al. 2000; Marsch 2005). Auch Lymphfisteln und Lymphzysten können als Komplikationen bei einem Lymphödem vorkommen. Die Fistelung erfolgt meistens kutan, aber auch lymphovaginale, lymphoanale und lymphorektale Fisteln können entstehen. Sie bergen dann, aufgrund der angrenzenden Strukturen, ein noch größeres Risiko für schwerwiegende Infektionen (Földi et al. 2002).

Eine seltene, jedoch umso schwerwiegendere Komplikation stellt das Lymphangiosarkom (Stewart-Treves-Syndrom) dar. Es entsteht häufig bei chronischen Lymphödemen, die unzureichend oder schlecht therapiert wurden und somit die Bildung von atrophischer, verdickter, hyperkeratotischer Haut im entsprechenden Bereich zur Folge haben (Felmerer et al. 2016; Melsom et al. 1981; Giannardi et al. 1960). Beim Lymphangiosarkom handelt es sich um ein Weichteilsarkom der Haut, welches nach operativer Therapie eines Mammakarzinoms mit axillärer Lymphknotendissektion entstehen kann (Stewart und Treves 1948; Felmerer et al. 2016). Als Ursprungszellen dienen Epithelzellen der Blutgefäße (Goldblum et al. 2014). Visuell ähneln sie einem banalen Hämatom, was jedoch sorgfältig klinisch und histologisch abgeklärt werden muss (Felmerer et al. 2016). Ausgehend von den initial unscheinbaren Veränderungen bilden sich im weiteren klinischen Verlauf Plaques oder Noduli, die sich in Nekrosen, Ulzerationen und Blutungen ausweiten können (Stewart und Treves 1948). Die Therapie der Wahl ist die frühzeitige, radikale Exzision, sodass dem aggressiven Wachstumsmuster und der frühen Metastasierung entgegengewirkt werden kann (Stewart et al. 1995).

\subsubsection{Prävention}

Ziel der Primärprävention ist es der Erkrankung zuträgliche Verhaltensweisen zu erlernen und hinderliche oder schädigende Verhaltensweisen zu unterlassen (Fu et al. 2008; Fu et al. 2010; Sherman und Koelmeyer 2011; Leitlinie Lymphödem 2017). Der Primärprävention sollte durch intensive Patientenschulung eine besondere Rolle zukommen.

Betroffene Personen sollten beispielsweise passendes, weiches Schuhwerk tragen und einschnürende Kleidung vermeiden. Weiter sollte auf penible Fußpflege geachtet werden, um die Eintrittspforten für Erysipele so niedrig wie möglich zu halten und Verletzungen und Überanstrengung, wie auch starke Hitze oder Sonnenbrand, auf ein Minimum zu 
reduzieren. Auch Kälte sowie langes Stehen, Gehen oder Insektenstiche sollten vermieden werden (Dupuy et al. 1999; Földi und Földi 2002b). Ein normwertiges Körpergewicht sowie eine ausgewogene Ernährung mit regelmäßiger Bewegung sollte angestrebt werden (Paskett et al. 2007). Komorbiditäten, wie eine chronisch venöse Insuffizienz oder rheumatoide Arthritis, sollten leitliniengerecht behandelt werden (Eberhardt und Raffetto 2014). Bei Entzündungszeichen sollte sofort ein Arzt konsultiert werden (Chlebicki und Oh 2014). Zusätzlich zur Schulung des Patienten werden auch therapeutische Aspekte zur Primärprävention gezählt. So kann heutzutage, durch die feste Etablierung der SentinelLymphknoten Biopsie, häufig auf eine komplette Lymphknotenentfernung verzichtet werden. Dadurch reduziert sich das Risiko der Entwicklung postoperativer Lymphödeme (Füller et al. 2008; Mansel et al. 2006).

Für die Sekundärprävention sind alle bereits in der Primärprävention genannten Faktoren von besonderer Wichtigkeit. Hinzu kommt das konservative Therapieprozedere im Sinne einer Komplexen Physikalischen Entstauungstherapie (KPE) (Leitlinie Lymphödem 2017; International Society of Lymphology 2013; Földi et al. 2000).

Die Tertiärprävention dient der Verhinderung des Fortschreitens der Erkrankung und soll der Entstehung von Komplikationen entgegenwirken. Im Bezug auf das chronische Lymphödem sind hier das Verhindern von Erysipelen und des Stewart-Treves-Syndroms von äußerster Wichtigkeit (Leitlinie Lymphödem 2017; Felmerer et al. 2016; Chlebicki und Oh 2014).

\subsubsection{Stadieneinteilung}

Das Lymphödem wird nach der International Society of Lymphology (ISL) in vier Stadien eingeteilt. Entsprechende Stadien mit klinischer Erscheinung und Charakterisitika sind in Tabelle $1 \mathrm{zu}$ sehen. 
Tabelle 1: Stadieneinteilung des Lymphödems nach der ISL (International Society of Lymphology 2013; Leitlinie Lymphödem 2017).

\begin{tabular}{|l|l|}
\hline Stadium & Klinische Erscheinung und Charakteristik \\
\hline Stadium 0 & $\begin{array}{l}\text { Latenzstadium; kein klinisch apparentes Lymphödem; pathologisches } \\
\text { Lymphszintigramm möglich; subklinisches Stadium }\end{array}$ \\
\hline Stadium I & $\begin{array}{l}\text { Ödem von weicher Konsistenz; Eindrückbarkeit kann vorhanden sein; } \\
\text { Hochlagern reduziert die Schwellung; spontan reversibel }\end{array}$ \\
\hline Stadium II & $\begin{array}{l}\text { Ödem mit sekundären Gewebeveränderungen; Hochlagerung beseitigt die } \\
\text { Schwellung nicht; nicht spontan reversibel }\end{array}$ \\
\hline Stadium III & $\begin{array}{l}\text { Elephantiasis; deformierte harte Schwellung, zum Teil lobäre Form, zum Teil mit } \\
\text { typischen Hautveränderungen }\end{array}$ \\
\hline
\end{tabular}

\subsection{Diagnostik}

Bei fehlenden oder nicht wesentlichen Begleiterkrankungen kann die Diagnosestellung eines Lymphödem mithilfe der klinischen Basisdiagnostik erfolgen. Hierzu gehört eine ausführliche Anamnese, gründliche Inspektion sowie sorgfältige Palpation. In der Regel kann dann, mit Hilfe des klinischen Befundes, die Stadieneinteilung des Lymphödems erfolgen (Leitlinie Lymphödem 2017; International Society of Lymphology 2013; Baumeister 2009). Zur Unterscheidung, ob es sich um ein primäres oder sekundäres Lymphödem handelt, ist vor allem der zeitliche Beginn und damit in Verbindung stehende Ereignisse, wie Operationen, zu berücksichtigen. Auch bereits durchgeführte Therapien und die erstmalige Diagnosestellung sind relevante Informationen. Bei der Inspektion ist zu klären, ob es sich um eine einseitige oder beidseitige Schwellung handelt und, ob eine Symmetrie vorliegt. Für die Stadieneinteilung nach ISL ist von besonderer Wichtigkeit ob die Ödeme beim Hochlagern noch reversibel sind. Die Eindrückbarkeit und somit Einschätzung der bereits stattgefundenen fibrosklerotischen Veränderungen wird bei der Palpation getestet. Hierbei sollte auch das Stemmersche Hautfaltenzeichen geprüft und das Vorhandensein von Kastenzehen untersucht werden. Je nach initialem klinischem Untersuchungsbefund ist dann die weitere Diagnostik notwendig. Hierbei sind organmorphologische Ursachen für das Lymphödem $\mathrm{zu}$ eruieren und relevante Begleiterkrankungen, wie beispielsweise das Lipödem, zu diagnostizieren (Leitlinie Lymphödem 2017; Földi et al. 2002; Baumeister 2009; International Society of Lymphology 2013). 
Zusätzlich, zu der oben genannten Basisdiagnostik, sind verschiedene apparative Verfahren zur Differentialdiagnostik möglich (International Society of Lymphology 2013). Im Folgenden sollen einige Verfahren, ohne Anspruch auf Vollständigkeit, vorgestellt werden. Zu erwähnen ist hierbei, dass viele Verfahren noch aktueller Teil der Forschung sind und durch Weiterentwicklung der technischen Möglichkeiten einem stetigen Wandel unterliegen.

\subsubsection{Umfangs- und Volumenmessung}

Eine verlässliche und unkomplizierte Methode die Umfänge einer Extremität zu messen ist unter Zuhilfenahme eines Maßbandes möglich. Hierbei wird eine festgelegte Anzahl von Messpunkten an der zu erhebenden Struktur definiert und diese dann mit dem Maßband abgemessen (Blaney et al. 2015; Herpertz 2010; Sawan et al. 2009).

Die einfachste Methode eine Volumenmessung durchzuführen ist mit Hilfe der Wasserverdrängungsmethode. Dabei wird das zu messende Objekt in ein mit Wasser gefülltes Becken gestellt. Unter Berücksichtigung des Volumens im Becken sowie des Wertes vor dem Eintauchen, kann der Volumenwert der Extremität berechnet werden (Herpertz 2010; Kaulesar Sukul et al. 1993). Eine weitere Möglichkeit das Volumen zu bestimmen stellt die Perometrie dar. Sie ist ein optoelektrisches Messverfahren, bei dem ein Gerät, bestehend aus Rahmen und zwei Infrarotstrahlern, die Extremität durch Bewegung abtastet und daraus das entsprechende Volumen errechnet (Jain et al. 2010).

\subsubsection{Lymphszintigraphie}

Bei der Lymphszintigraphie unterscheidet man prinzipiell zwei Verfahren. Zum einen die statische, qualitative Lymphszintigraphie, zum anderen die quantitative Funktionslymphszintigraphie (Brauer und Weissleder 2017). Beide Verfahren beginnen mit der subkutanen Injektion von radioaktiv markiertem Humanalbumin in den Fußrücken, beziehungsweise Handrücken. Dieses wird aufgenommen und über die Lymphgefäße in den entsprechenden regionalen Lymphknoten transportiert. Hier findet eine Anreicherung statt. Bei der Funktionslymphszintigraphie erfolgt direkt nach der Injektion, und über einen definierten Zeitraum, eine verlaufsartige Messung, sowie eine Messung der Anreicherung in den regionalen Lymphknoten. Dies erfolgt mit einer Gammakamera (Brauer und Weissleder 2017; Tiedjen et al. 2002). Da Injektionszeit und Ankunftszeit im Lymphknoten bekannt beziehungsweise darstellbar sind, kann daraus die Transportgeschwindigkeit berechnet werden. Die langsamere Aufnahme und die verlängerte Transportzeit zum 
Lymphknoten sind Hinweise auf eine Lymphabflussstörung (Brauer und Weissleder 2017; Brauer 1996). Nach der Funktionslymphszintigraphie kann eine qualitative Lymphszintigraphie durchgeführt werden. Hier wird zwischen Ganzkörper- und Teilkörperaufnahmen unterschieden (Brauer und Weissleder 2017). Da sich in dieser Aufnahme Lymphkollektoren und Lymphknoten in ihrer Gesamtheit darstellen lassen, gibt die Untersuchung Aufschluss über entsprechende anatomische Veränderungen. Häufig sind hier, bei Lymphödempatienten, eine verringerte Anzahl von Lymphkollektoren und Lymphknoten erkennbar (Brauer und Weissleder 2017). Klassischerweise kommt es dann zur Umverteilung und dem oberflächlichen Abfluss der Lymphe über das umliegende Gewebe und die Haut (Sty et al. 1979; Dylke et al. 2013).

\subsubsection{Indocyaningrün-Lymphographie}

Eine weitere diagnostische Möglichkeit stellt die Indocyaningrün-Lymphographie (ICGL) dar. Hierbei wird der fluoreszierende Farbstoff Indocyaningrün in den Vorfuß des Patienten gespritzt. Nach kurzer Resorptionszeit können die oberflächlichen Lymphgefäße der Haut, mit Hilfe einer Infrarotkamera, dargestellt werden. Als pathologische Fluoreszenzmuster unterscheidet man ein lineares Muster von einem Fluoreszenzmuster des dermal backflows. Während das lineare Muster häufig in den frühen Stadien des Lymphödems vorkommt, so ist der dermal backflow eher bei fortgeschrittenen Stadien zu sehen (Yamamoto et al. 2015; Yamamoto et al. 2011b; Unno et al. 2010; Unno et al. 2007).

\subsubsection{Kernspinlymphangiographie und Computertomographie}

Bei der Kernspintomographie lässt sich, durch die verschiedenen Wichtungen, das Weichteilgewebe sowie auch Lymphgefäße und Flüssigkeitsansammlungen besonders gut darstellen ( $\mathrm{Lu}$ et al. 2010; Mitsumori et al. 2015). Die Diagnostik beginnt mit dem intrakutanen Einspritzen von wasserlöslichem Kontrastmittel in die Zehenzwischenräume. Danach erfolgt die Bildgebung in festgelegten Zeitintervallen (Weiss et al. 2014; Mitsumori et al. 2015). Durch die sich in den Zeitintervallen verändernde Kontrastmittelaufnahme, beziehungsweise dessen Weitertransport, können alle lymphatischen Strukturen, von Initialgefäßen bis Lymphknoten, dargestellt werden. Zusätzlich gibt die Bildgebung Aufschluss über Flussgeschwindigkeit und Leistungsvermögen des Lymphsystems. Auch Pathologien wie Lymphostase, mit dem sich entwickelnden Phänomen des dermal backflows, können festgestellt werden (Mitsumori et al. 2015; Notohamiprodjo et al. 2009; Liu et al. 2009). Eine Diskussion der Kernspinlymphangiographie als Alternative zur Lymphszintigraphie wird in verschiedenen Studien sowohl präoperativ als auch zur 
Verlaufskontrolle und Erfolgsquantifizierung postoperativ diskutiert (Mitsumori et al. 2015; Lohrmann et al. 2009; Lohrmann et al. 2006).

Der Computertomographie kommt in der Lymphödemdiagnostik eine eher geringe Rolle zu. Die hohe Strahlenexposition sowie die deutlich schlechtere Auflösung im Bereich der Weichteile sind die Hauptgründe hierfür. Eine genaue Beurteilung der Lymphgefäße ist nicht möglich, weshalb sie vor allem in der Onkologie für abdominelle und retroperitoneale Tumordiagnostik mit Beteiligung der großen Lymphabflussbahnen ihre Daseinsberechtigung hat (Goyal et al. 2015; Tiedjen et al. 2002; Hadjis et al. 1985).

\subsubsection{Bioimpedanzmessung}

Die Bioimpedanzmessung, initial als Messmethode zur Bestimmung des Gesamtkörperwassers eingeführt (Hoffer et al. 1969), wird immer zahlreicher auch in der Lymphödemdiagnostik, eingesetzt (Erdogan Iyigun et al. 2015; Gaw et al. 2011; Warren et al. 2007; Cornish et al. 2001). Das grundlegende Funktionsprinzip der Messungen ist in den verschiedenen elektrischen Leitfähigkeiten der unterschiedlichen Geweben und Flüssigkeiten im Körper begründet (Cornish 2006). Beim Lymphödem kommt es zur Vermehrung extrazellulärer Flüssigkeit im Körper. Je nach Flüssigkeitsmenge im Gewebe wird einem hindurchgeleiteten Strom ein unterschiedlicher Widerstand entgegengesetzt. Dieser Wiederstand kann von Elektroden auf der Haut gemessen werden und wird Impedanz genannt. Somit ergeben verschiedenen Flüssigkeitsvolumina unterschiedliche Messwerte der Impedanz.

\subsubsection{Der prozentuale Wassergehalt der Haut und die Dielektrizitätskonstante}

Die Messung des prozentualen Wassergehaltes der Haut ist eine neuere Möglichkeit in der Lymphödemdiagnostik. Über eine Messsonde, die visuell einem Ultraschallkopf ähnelt, wird eine hochfrequente elektromagnetische Welle mit $300 \mathrm{MHz}$ in die Haut bis zum subkutanen Fettgewebe gesendet (Nuutinen et al. 2004). Auf dem Weg durch die Hautschichten wird das elektromagnetische Feld teilweise, von im Körper befindlichen Wassermolekülen, absorbiert (Alanen et al. 1999; Pennock und Schwan 1969). Der nicht absorbierte Teil wird reflektiert und kann an der Messsonde detektiert werden. Durch die vorhandenen zwei Werte kann die Polarisierbarkeit der Haut berechnet und die Dielektrizitätskonstante ermittelt werden (Stuchly und Stuchly 1980). Sie ist direkt proportional zum Wassergehalt in dem gemessenen Areal (Nuutinen et al. 2004). 


\subsection{Therapie}

\subsubsection{Konservative Therapie}

Beim Lymphödem handelt es sich um eine chronisch progrediente Erkrankung, daher ist der Beginn der konservativen Therapie zeitnah nach Diagnosestellung von äußerster Wichtigkeit. Aufgrund der Tatsache, dass das Lymphödem zum aktuellen Zeitpunkt nicht heilbar ist, kommen der Linderung des Leidensdrucks sowie der Ödemreduktion ein wichtiger Stellenwert zu. Im Optimalfall kann eine Stadienreduktion bis zum Latenzstadium erreicht werden (Leitlinie Lymphödem 2017; Földi et al. 2002). Bei langem Bestehen des Lymphödems kommt es im Verlauf zur Bildung von fibrosklerotischem Gewebe. Umso fortgeschrittener der Umbau bereits ist, desto komplizierter und länger dauert die Reduktion des entsprechenden Gewebes.

Die bereits 1892 von Winiwarter beschriebene Komplex Physikalische Entstauungstherapie (KPE) stellt die zentrale Säule des konservativen Therapieprozederes dar (Winiwarter 1892). Sie unterteilt sich in zwei Phasen und vier Maßnahmen: manuelle Lymphdrainage (MLD) nach Vodder (Vodder 1936, 1983), Kompressionstherapie, entstauende Bewegungstherapie und Hautpflege. Die Phase I der KPE dauert, unter Berücksichtigung der Ausprägung des Ödems, bis zu vier Wochen und beinhaltet bedarfsgerechte MLD, 24stündige Bandagierung, regelmäßige tägliche Bewegungstherapie und Hautpflege. Ein weiteres wichtiges Element stellt die ausführliche Patientenschulung dar. Im Anschluss an die Phase I wird die Phase II durchgeführt. Sie beinhaltet die Weiterführung der bedarfsgerechten MLD, das konsequente Tragen eines Kompressionsstrumpfes, tägliche Bewegungstherapie und gründliche Hautpflege. In der Phase II ist der betroffene Patient in der Eigenverantwortung die zuvor erlernten Methoden und Maßnahmen umzusetzen (Leitlinie Lymphödem 2017; International Society of Lymphology 2013; Földi et al. 2002; Földi et al. 1985).

Bei besonders leicht ausgeprägten Ödemen kann ein Auslassversuch oder Ausschleichen probeweise durchgeführt werden. Normalerweise muss die Kompressionsbestrumpfung aber ein Leben lang getragen und auch regelmäßig MLD durchgeführt werden. Bei sehr stark ausgeprägten Ödemen kann es sinnvoll und notwendig sein eine regelmäßige akute Entstauungstherapie, im Sinne einer KPE Phase 1, durchzuführen (Földi et al. 2002; Kasseroller 1998). Unter Abwägung der individuellen Vorerkrankungen und Kontraindikationen des Patienten ist die KPE risikofrei. Als Kontraindikationen gelten: eine akute Infektion oder Entzündung im Sinne eines Erysipels oder eine akute 
Thrombophlebitis, eine dekompensierte Herzinsuffizienz (Wilputte et al. 2005; Todd et al. 2011), eine periphere arterielle Verschlusskrankheit (pAVK), Phlebothrombose oder ein Malignom im Lymphabstromgebiet der entsprechenden Extremität (Leitlinie Lymphödem 2017). Zusätzliche Maßnahmen zur KPE sind intermittierende pneumatische Kompressionstherapie, Bewegungstherapie im Wasser oder Wärme- und Kältetherapie (Leitlinie Lymphödem 2017; International Society of Lymphology 2013; Földi et al. 2002).

\subsubsection{Medikamentöse Therapie}

Die Suche nach einer direkten medikamentösen Therapie ist zum aktuellen Zeitpunkt noch Gegenstand von klinisch experimentellen Untersuchungen. In unterschiedlichen Versuchen konnte bereits gezeigt werden, dass die Pumpfunktion und dadurch die Transportkapazität der Lymphgefäße durch lokale Applikation von Histamin, Dopamin oder Serotonin gesteigert werden kann (Földi et al. 2002). Weitere Studien untersuchen den Einfluss von lokal applizierten entzündungshemmenden Medikamenten, wie Ketoprofen, Selen oder Tacrolimus, auf den klinischen Verlauf des Lymphödems (Forte et al. 2019). Obwohl über die Jahre immer wieder vielversprechende Entdeckungen gemacht wurden, so konnte sich bis heute keine medikamentöse Therapie standardmäßig etablieren. Besonders eine Therapie mit Diuretika sollte nur bei entsprechenden Komorbiditäten durchgeführt und nicht als kausale Therapie des Lymphödems angewandt werden (Földi et al. 1985; Leitlinie Lymphödem 2017).

Eine antibiotische Therapie sollte nur bei der Behandlung von Komplikationen, wie Erysipelen, eingesetzt werden. Eine prophylaktische antibiotische Abdeckung ist nur in wenigen Fällen und nach strenger Indikationsprüfung sinnvoll (Chlebicki und Oh 2014).

\subsubsection{Chirurgische Therapie}

Die ersten dokumentierten chirurgischen Therapieversuche starteten Anfang des 20. Jahrhunderts. Grundlage dieser frühen operativen Methoden war es mit Hilfe von subkutan verankerten Seidenfäden den Lymphabfluss zu verbessern (Handley 1908, 1910), oder durch Fenestrierung der Muskelfaszien und Knochen einen Abfluss der epifaszialen Lymphflüssigkeit in intramuskuläre oder subperiostale Lymphgefäße zu ermöglichen (Lanz 1911). Diese ambitionierten Möglichkeiten der Lymphödemtherapie zeigten sich fast allesamt erfolglos. Parallel dazu wurde 1912 von Sir Havelock Charles ein radikales Verfahren zur Therapie des elephantiastischen Lymphödems beschrieben (Charles 1912). Hierbei wurde am entsprechenden Bein eine radikale epifasziale Resektion des Gewebes 
durchgeführt. Im Anschluss erfolgte eine Spalthautdeckung der zurückgebliebenen Faszie. Noch heute ist dieses Verfahren unter dem Namen „Charles Procedure“ bekannt (Charles 1912; Dumanian und Futrell 1996). Ähnlich wie Lanz 1911 mit seiner Fenestrierung der Muskelfaszie, so versuchte auch Emmanuil Kondoleon, mit einem weitaus invasiverem Verfahren, Lymphödeme zu therapieren (Dimakakos et al. 2009). Seine Idee war es eine Kommunikation zwischen epifaszialer und subfaszialer Lymphe zu schaffen und somit einen suffizienten Lymphabfluss zu ermöglichen. Um dies zu gewährleisten resezierte er großflächige Faszienstreifen medial und lateral der entsprechenden Extremität sowie auch fibrosklerotisch verändertes Gewebe (Kondoleon 1912a, 1912b; Weinstein und Roberts 1950). Bis zur Etablierung der Mikrochirurgie Mitte des 20. Jahrhunderts waren das „Kondoleon Procedure" sowie auch der oben genannte Ansatz von Charles die probatesten chirurgischen Verfahren in der Lymphödemtherapie (Dimakakos et al. 2009).

Auch heute noch wird besonders das „Charles Procedure“, in modifizierter Form, von einigen Chirurgen angewandt (Karri et al. 2011; Sapountzis et al. 2014b). Im Allgemeinen wurden diese radikalen resezierenden Verfahren jedoch, aufgrund der deutlich erhöhten Risiken und Komplikationen, von den mikrochirurgischen Verfahren abgelöst.

Die heutigen Therapiemöglichkeiten bei der operativen Versorgung des Lymphödems unterteilen sich in mikrochirurgisch-rekonstruktive, deviierende sowie resezierende Verfahren (International Society of Lymphology 2013; Leitlinie Lymphödem 2017). Zu den mikrochirurgisch-rekonstruktiven Verfahren werden die Interposition autologer Venen sowie auch die autologe Lymphgefäßtransplantation gezählt. Deviierende Verfahren beinhalten die Anlage von lympho-venöser Anastomosen, lymphonodulo-venöse Anastomosen und die autologe Lymphknotentransplantation. $\mathrm{Zu}$ den bereits weiter oben beschriebenen Möglichkeiten der resezierenden Verfahren zählt auch die Liposuktion.

Im Folgenden soll hauptsächlich auf die Lymphgefäßtransplantation und die Lymphknotentransplantation eingegangen werden.

Prinzipielles Ziel jeglicher chirurgischer Eingriffe sollte die Senkung des Leidensdrucks, die Reduktion der Funktionseinschränkung der entsprechenden Extremität sowie die Reduktion des Ödems, wenn möglich bis ins Latenzstadium, sein (Leitlinie Lymphödem 2017; Földi und Földi 2002a). Die präoperative Vorbereitung, im Sinne von konsequent durchgeführter und ausgereizter KPE über mindestens sechs Monate, sollte gegeben sein (Leitlinie Lymphödem 2017). Weiter muss der Patient gründlich und realistisch über das postoperative Ergebnis, die zu erwartende Verbesserung und die eventuelle Notwendigkeit der Weiterführung der konsequenten Therapie aufgeklärt werden. 


\subsubsection{Liposuktion}

Die Liposuktion stellt ein in den 1980er Jahren von Yves-Gerard Illouz für die ästhetische Chirurgie entwickeltes Verfahren dar, welches dem Chirurgen ermöglicht eine große Anzahl von subkutan gelegenem Fett in einem Eingriff abzusaugen (Illouz 1980, 1983). Negative Auswirkungen des Eingriffes auf die Lymphtransportkapazität des Lymphsystems konnten nicht bestätigt werden (Brorson et al. 1998). Über die Jahre hinweg entwickelten sich eine Vielzahl von zusätzlichen Möglichkeiten den Prozess der Liposuktion noch schonender und nebenwirkungsärmer zu machen. So wurden Verfahren mit Vibration-, Laser- oder Ultraschallunterstützung etabliert.

Ab 1987 wurde die Liposuktion auch in der Therapie des chronischen Lymphödems eingesetzt (Brorson und Svensson 1998; O'Brien et al. 1989). Voraussetzung für das erfolgreiche Gelingen ist eine konsequent, vor und nach dem Eingriff, durchgeführte konservative Therapie. Ein gutes Bild über die Ausschöpfung der konservativen präoperativen Therapie gibt die Eindrückbarkeit in der prätibialen Region der entsprechenden lymphödematös geschwollenen Extremität. Zeigt sich hier eine tiefe Eindrückbarkeit ist dies ein Zeichen dafür, dass die konservative Therapie noch nicht vollends ausgeschöpft ist (Brorson et al. 2008a; Brorson et al. 2008b).

Nach Brorson kann eine Liposuktion beim chronischen Lymphödem nur dann erfolgversprechend und von Dauer sein, wenn das Tragen der Kompressionsbestrumpfung postoperativ für 24 Stunden durchgeführt wird (Brorson et al. 2008a; Brorson et al. 2008b; Brorson und Svensson 1998).

Zusätzlich sind in den letzten Jahren vermehrt Therapieansätze vorgestellt worden, welche die Liposuktion mit mikrochirurgischen Verfahren kombinieren, um noch bessere Ergebnisse zu erzielen (Nicoli et al. 2015; Cook et al. 2016).

\subsubsection{Autologe Lymphgefäßtransplantation}

Die autologe Lymphgefäßtransplantation im Bereich der unteren Extremität wurde von dem deutschen Chirurgen Rüdiger Baumeister erstmalig im Jahre 1981 durchgeführt (Baumeister et al. 1986; Baumeister et al. 1981b; Baumeister et al. 1981a). In den folgenden Jahren wurde diese Operationsmethode dann auch an der oberen Extremität, bei Patienten mit Armlymphödem nach Mastektomie, angewandt (Baumeister 1983; Baumeister et al. 1986). In der chirurgischen Lymphödemtherapie gilt dieses Verfahren seitdem als fest etabliert. 
Die Lymphgefäßtransplantation kann bei einseitig lokalisiertem Lymphödem, nach operativem tumorchirurgischem Eingriff, erfolgen. Eine Schwellneigung im Bereich der kontralateralen Seite ist ein Ausschlusskriterium für den Eingriff, ebenso wie eine aktive Tumorerkrankung (Felmerer et al. 2012; Baumeister et al. 1986). Zusätzlich sollte eine konservative Therapie für mindestens sechs Monate präoperativ erfolgt sein (Baumeister 1983).

Zur Visualisierung der Lymphkollektoren, und dadurch Vereinfachung der Präparation dieser, werden präoperativ ungefähr $2 \mathrm{ml}$ Patentblau zwischen die erste und zweite Kommisur der Zehen des gesunden Beines intradermal und subkutan injiziert. Dies kann unsteril, aber nach gründlicher Desinfektion des entsprechenden Bereiches, erfolgen und ermöglicht die Aufnahme und den Abtransport des Farbstoffes während der restlichen präoperativen Maßnahmen. Im Anschluss erfolgt die Darstellung mehrerer Lymphkollektoren des ventromedialen Bündels auf der gesunden Seite sowie die Präparation der Anschlussgefäße auf der Seite des Lymphödems. Dies erfolgt in der Regel mit Lupenbrille. Von den insgesamt circa 15-20 vorliegenden Gefäßen werden zwei bis drei auf einer Länge von 25-30 cm herauspräpariert und distal ligiert. Proximal bleiben die Lymphgefäße mit den inguinalen Lymphknoten verbunden (Felmerer et al. 2012). Die Lymphgefäße der gesunden Seite werden dann im Bereich der Symphyse auf die ödematöse Seite getunnelt (Baumeister et al. 1986). Hier erfolgt die mikrochirurgische Anastomose mit den epifaszialen, von distal kommenden, Lymphkollektoren (Felmerer et al. 2012). Bei sorgfältiger Lymphgefäßpräparation ist die Entstehung eines sekundären Lymphödems auf der Spenderseite gering (Felmerer et al. 2012).

Eine perioperative Antibiose mit Weiterführung bis ungefähr drei Wochen postoperativ sollte zur Vermeidung von Wundinfektionen und Erysipelen durchgeführt werden (Felmerer et al. 2012).

\subsubsection{Autologe Lymphknotentransplantation}

In den 1980er Jahren führte Corinne Becker erstmals eine autologe Lymphknotentransplantation bei Patientinnen nach Mammakarzinom und konsekutiver Axilladissektion durch (Becker et al. 2012; Becker et al. 2006; Becker et al. 1991). In den darauffolgenden Jahren entwickelte sie die operative Technik weiter, sodass die entnommenen Lymphknotenpakete auch in der Therapie des Beinlymphödems eingesetzt werden konnten (Batista et al. 2017; Batista et al. 2013). Zum aktuellen Zeitpunkt werden verschiedene Techniken und Methoden angewendet, wodurch eine Vielzahl von Varianten in der Auswahl des Entnahme- sowie Transplantatortes bestehen. Prinzipiell werden aber 
immer vaskularisierte Lymphknotenpakete, jeweils mit oder ohne eine Hautinsel, aus einer gesunden, ödemfreien Region in den entsprechenden lymphödematösen Bereich transferiert. Als potentielle Spenderregion sind die supraklavikuläre- (Becker et al. 2012; Sapountzis et al. 2014a), submentale- (Cheng et al. 2012), axilläre- (Silva und Chang 2016), thorakale- (Becker et al. 2012), inguinale- (Lin et al. 2009; Cheng et al. 2013) und omentale Spenderregion (Silva und Chang 2016; Nguyen und Suami 2015) beschrieben worden.

Bei der Empfängerregion wird in anatomische Regionen und nichtanatomische Regionen unterschieden. Anatomische Regionen sind die Axilla oder die Leiste. Als nichtanatomische Region gelten beispielsweise die Implantation in der Knöchelregion, Kniekehle oder am Handgelenk (Silva und Chang 2016). 


\section{Material und Methoden}

\subsection{Patientenkollektiv}

Im Rahmen dieser Dissertationsarbeit wurden Daten von drei verschiedenen Kollektiven erhoben und ausgewertet. Insgesamt konnten 87 Patienten, die im Zeitraum von März 2010 bis April 2020 im Schwerpunktbereich der Plastischen Chirurgie der Klinik für Unfallchirurgie, Orthopädie und Plastische Chirurgie der Universitätsmedizin Göttingen behandelt wurden, eingeschlossen werden.

Die Auswertung beinhaltet Daten, welche routinemäßig bei jeder Vorstellung in der Sprechstunde, beziehungsweise bei operativer Versorgung, erhoben wurden. Des Weiteren liegt eine Genehmigung mit Ethikvotum der Ethikkommission vom 15.07.2015, Antragsnummer 10/10/14 vor.

\subsubsection{Autologe omentale Lymphknotentransplantation roboterassistiert}

Das Patientenkollektiv dieser Untersuchungsgruppe beinhaltete Patienten, die sich im Zeitraum von November 2017 bis April 2020 in unserem Schwerpunktbereich der Plastischen Chirurgie in Behandlung befanden, beziehungsweise in diesem Zeitraum eine operative Versorgung, bestehend aus der Transplantation von omentalen Lymphknoten in die entsprechende lymphödematös veränderte Extremität, erhielten. In die Auswertung der Umfangsmessung wurden Patienten eingeschlossen, deren Operationstermin im April 2020 mindestens 12 Monate zurücklag.

Einschlusskriterien zur operativen Versorgung waren Infektions- und Entzündungsfreiheit am Tag der operativen Versorgung. Bei sekundären Lymphödemen aufgrund von Tumorleiden war eine dauerhafte Remission mit regelhaften onkologischen Verlaufskontrollen notwendig. Um Patienten mit unvollständiger konservativer Therapie herauszufiltern, wurde ein vollständiges konservatives Therapieprocedere für sechs Monate präoperativ als Bedingung festgelegt. Bei Auffälligkeiten im klinischen Untersuchungsbefund, die auf eine unvollständige Entstauung hinwiesen, wurde zur Durchführung einer stationären Reha mit Komplex Physikalischer Entstauung (KPE) Phase 1 vor operativer Versorgung geraten. Die operative Versorgung erfolgte dann nach abgeschlossener KPE Phase 1. 
Insgesamt konnten 18 Patienten in die Studie eingeschlossen werden (ein Mann, 17 Frauen). Das Durchschnittsalter der Patienten lag am Tag der operativen Versorgung bei 57,45 \pm 8,02 Jahren (Bereich: 40-75 Jahre), der mittlere Beobachtungszeitraum bei $18 \pm$ 3,48 Monaten (Bereich: 12-27 Monate).

In die Auswertung der Umfangswerte sowie des Bedarfes an postoperativer konservativer Therapie konnten elf Patienten eingeschlossen werden.

In den Anamnesegesprächen wurde besonderes Augenmerk auf die Ursachen, die Latenzzeit bis zum Auftreten des Ödems, das Maß an durchgeführter konservativer Therapie sowie die Frage nach Erysipelen oder anderen Komplikationen gelegt. Postoperativ war das anamnestische Erfragen von gastrointestinaler Beschwerdesymptomatik sowie weiterer Beschwerden oder Komplikationen essentiell. Ein notwendiges Einschlusskriterium für den Eingriff war die im initialen Eingriff stattgefundene Entfernung der entsprechenden Lymphknoten im Bereich der inguinalen beziehungsweise axillären Region. Patienten mit entfernten pelvinen oder paraaortal Lymphknoten, sowie der alleinigen Entfernung des Sentinel-Lymphknotens nach Mammakarzinom, wurden nicht eingeschlossen und auch nicht operiert. Diesen Patienten wurde bei Operationswunsch und separater Indikationsstellung eine Lymphgefäßtransplantation oder die Anlage von lymphovenösen Anastomosen (LVA) angeboten.

Intraoperativ erfolgte die zeitliche Erfassung und Dokumentation der einzelnen Operationsschritte.

Postoperativ stellten die Patienten sich in regelmäßigen Abständen in der Sprechstunde der Plastischen Chirurgie der Universitätsmedizin Göttingen vor. 


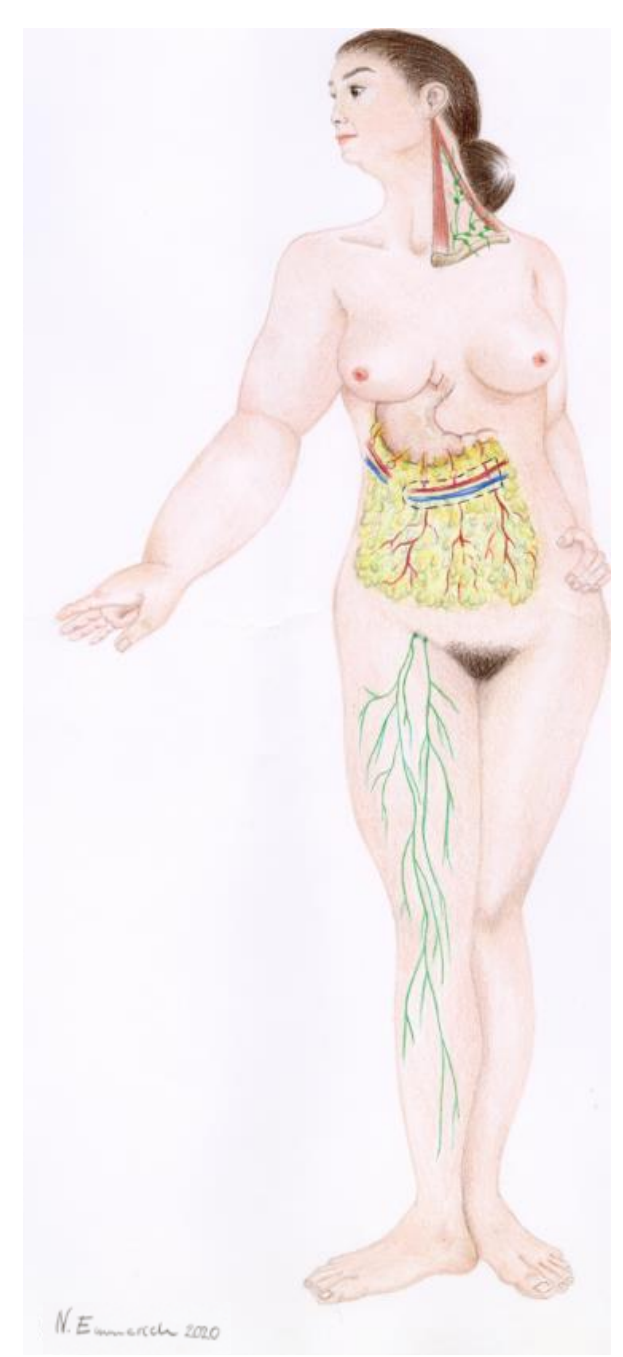

Abbildung 1: Grafische Darstellung der Spenderregionen der einzelnen Eingriffe. Die Verwendung erfolgt mit freundlicher Genehmigung der Zeichnerin N. Emmerich.

\subsubsection{Autologe zervikale Lymphknotentransplantation}

Das Patientenkollektiv dieser Untersuchungsgruppe beinhaltete Patienten, die sich im Zeitraum von Januar 2013 bis April 2019 in unserem Schwerpunktbereich der Plastischen Chirurgie der Universitätsmedizin Göttingen vorstellten, beziehungsweise in diesem Zeitraum eine operative Versorgung bestehend aus der Transplantation von zervikalen Lymphknoten in die entsprechende lymphödematös veränderte Extremität bekamen.

Einschlusskriterien für den Eingriff waren Infektions- und Entzündungsfreiheit am Tag der operativen Versorgung. Bei sekundären Lymphödemen aufgrund von Tumorleiden war eine dauerhafte Remission mit regelhaften onkologischen Verlaufskontrollen notwendig. Um eventuelle Patienten mit unvollständiger konservativer Therapie herauszufiltern, wurde ein vollständiges konservatives Therapieprocedere für 6 Monate präoperativ als Bedingung festgelegt. Bei Auffälligkeiten im klinischen Untersuchungsbefund, die auf eine unvollständige Entstauung hinwiesen, wurde zur Durchführung einer stationären Reha mit 
Komplex Physikalischer Entstauung Phase 1 vor operativer Versorgung geraten. Die operative Versorgung erfolgte dann nach abgeschlossener KPE Phase 1.

Insgesamt konnten 33 Patienten in die Studie eingeschlossen werden (zwei Männer, 31 Frauen). Das Durchschnittsalter der Patienten am Tag der operativen Versorgung lag bei 49,76 \pm 4,16 Jahren (Bereich: 22-77 Jahre), der mittlere Beobachtungszeitraum bei 14,15 \pm 4,9 Monaten (Bereich: 4-66 Monate).

In die Auswertung der Operationszeiten wurden alle 33 Patienten, beziehungsweise deren Zeitwerte eingeschlossen.

In den Anamnesegesprächen wurde besonderes Augenmerk auf die Ursachen, die Latenzzeit bis zum Auftreten des Ödems, das Maß an durchgeführter konservativer Therapie sowie die Frage nach Erysipelen und anderen Komplikationen gelegt. Ein notwendiges Einschlusskriterium für den Eingriff war die, im initialen Eingriff, stattgefundene Entfernung der entsprechenden Lymphknoten im Bereich der inguinalen beziehungsweise axillären Region. Patienten mit entfernten pelvinen oder paraaortal Lymphknoten, sowie der alleinigen Entfernung des Sentinel-Lymphknotens nach Mammakarzinom, wurden nicht eingeschlossen und auch nicht operiert. Diesen Patienten wurde bei Operationswunsch und separater Indikationsstellung eine Lymphgefäßtransplantation oder die Anlage von lymphovenösen Anastomosen angeboten. Intraoperativ erfolgte die zeitliche Erfassung und Dokumentation der Operationszeit. Die genaue Dokumentation und Auswertung der einzelnen Operationsschritte entfiel hier.

Postoperativ stellten die Patienten sich in regelmäßigen Abständen in der PlastischChirurgischen Sprechstunde der Universitätsmedizin Göttingen vor.

\subsubsection{Lymphgefäßtransplantation}

Das Patientenkollektiv dieser Untersuchungsgruppe bestand aus Patienten, die sich im Zeitraum von März 2010 bis September 2018 in der Klinik für Unfallchirurgie, Orthopädie und Plastische Chirurgie der Universitätsmedizin Göttingen in Behandlung befanden, beziehungsweise in diesem Zeitraum eine operative Versorgung mittels der Transplantation von Lymphgefäßen erhalten hatten.

Einschlusskriterien für den Eingriff waren Infektions- und Entzündungsfreiheit am Tag der operativen Versorgung. Bei sekundären Lymphödemen aufgrund von Tumorleiden war eine dauerhafte Remission mit regelhaften onkologischen Verlaufskontrollen notwendig. Um eventuelle Patienten mit unvollständiger konservativer Therapie herauszufiltern, wurde 
ein vollständiges konservatives Therapieprozedere für 6 Monate präoperativ als Bedingung festgelegt. Bei Auffälligkeiten im klinischen Untersuchungsbefund, die auf eine unvollständige Entstauung hinwiesen, wurde zur Durchführung einer stationären Reha mit Komplex Physikalischer Entstauung Phase 1 vor operativer Versorgung geraten. Die operative Versorgung erfolgte dann nach abgeschlossener KPE Phase 1.

Insgesamt konnten 36 Patienten in dieses Patientenkollektiv eingeschlossen werden (acht Männer, 28 Frauen). Das Durchschnittsalter der Patienten bei operativer Versorgung lag bei 49,75 \pm 4,95 Jahren (Bereich: 15,9-60,7 Jahre), der mittlere Beobachtungszeitraum bei 14,84 \pm 4,46 Monaten (Bereich: 4-57 Monate).

In die Auswertung der Operationszeiten wurden alle Patienten beziehungsweise deren Zeitwerte eingeschlossen.

In den Anamnesegesprächen wurde besonderes Augenmerk auf die Ursachen, die Latenzzeit bis zum Auftreten des Ödems, das Maß an durchgeführter konservativer Therapie sowie die Frage nach Erysipelen oder anderen Komplikationen gelegt. Ein notwendiges Einschlusskriterium für den Eingriff war eine fehlende Schwellneigung im Bereich der Spenderregion, in der die Lymphgefäße entnommen werden sollten. Wenn die Patienten über eine entsprechende Schwellneigung nach Primäroperation berichteten, wurde keine Lymphgefäßtransplantation durchgeführt. Auch dann nicht, wenn bei Vorstellung in unserer Sprechstunde kein Lymphödem im Bereich des Spenderbeines bestand. Falls sich am Operationstag, nach präoperativer Lagerung und Einspritzen von Patentblau, ein dermal backflow im Bereich des Spenderbeines zeigte, wurde ebenfalls keine Lymphgefäßtransplantation vorgenommen. Es erfolgte daraufhin die Anlage von lymphovenösen Anastomosen (LVAs). Die Patienten wurden präoperativ über dieses Vorgehen aufgeklärt. In unserem Lymphgefäßkollektiv von 2010 bis 2018 trat dies einmalig auf. Es wurden bei der entsprechenden Patientin dann LVAs angelegt. Die Patientin wurde folglich nicht in dieses Patientenkollektiv aufgenommen.

Intraoperativ erfolgte die zeitliche Erfassung. Eine zeitliche Dokumentation der einzelnen Operationsschritte entfällt.

Postoperativ stellten sich die Patienten in regelmäßigen Abständen in der PlastischChirurgischen Sprechstunde der Universitätsmedizin Göttingen vor. 


\subsection{Diagnostik}

Wie zuvor in der Einleitung beschrieben, gibt es eine Vielzahl von Methoden zur Diagnose und Verlaufskontrolle des Lymphödems. Verlässliche Methoden sind besonders die Lymphszintigraphie, die Kernspinlymphographie, die Lymphographie und Umfangs- und Volumenmessungen. Alle Methoden, außer die Umfangs- und Volumenmessung, sind durch die radioaktiv markierten Stoffe oder Kontrastmittel deutlich aufwendigere Messverfahren. Daher wurden, neben der obligatorischen Anamnese sowie körperlicher Untersuchung, die Umfangsmessung mit Hilfe eines Maßbandes bei unserem Da-VinciPatientenkollektiv durchgeführt.

Alle diagnostischen oben beschriebenen Maßnahmen wurden jeweils vor dem chirurgischen Eingriff, nach sechs und nach zwölf Monaten postoperativ durchgeführt. Zu den entsprechenden Zeiten wurden die Patienten in die Sprechstunde der Plastischen Chirurgie einbestellt. Neben Wundkontrollen wurden auch klinische Verlaufskontrollen mit ausführlichen Gesprächen über postoperative Komplikationen angeboten. Weitere Termine, außerhalb der oben genannten Zeiten, waren individuell und nach Bedarf möglich.

Während des operativen Eingriffes erfolgte zusätzlich die Zeitmessung der entsprechenden operativen Einzelschritte.

Die Aufarbeitung des Supraklavikulären-/Zervikalen- und des Lymphgefäß-Kollektives wurde nach Aktenlage und ohne Erhebung der Umfangswerte durchgeführt.

\subsubsection{Umfangsmessung}

Die Umfangsmessung der jeweiligen Extremitäten erfolgt mit dem Maßband. Um eine Vergleichbarkeit zwischen den Messpunkten herzustellen und mögliche Veränderungen aufzuzeigen, wurden immer die gleichen Messpunkte verwendet. Für die untere Extremität waren dies der Mittelfuß, Knöchel, Unterschenkel, Knie und Oberschenkel. Am Unterund Oberschenkel wurden jeweils zwei Messpunkte genommen. Im Bereich der oberen Extremität wurde an Mittelhand, Handgelenk, Unterarm, Ellenbogen und Oberarm gemessen. Auch hier erfolgten am Unterarm und am Oberarm jeweils zwei Messungen.

\subsubsection{Operationszeiten}

Zur Auswertung der Veränderung der Operationszeiten mit dem Da-VinciOperationsroboter (Intuitive Surgical, Kalifornien, USA) wurden bei jedem Eingriff die gesamte Operationszeit sowie die Einsatzzeit des Da-Vinci-Systems protokolliert. Bei den 
anderen Operationen entfielen die einzelnen Operationsschritte. Hier wurde die gesamte Operationszeit durch die Schnitt-Naht Zeit im Operationsprotokoll erfasst.

\subsection{Therapie}

Alle Eingriffe wurden in Intubationsnarkose durchgeführt. Der erste sowie letzte Teil der Operation erfolgte mit Hilfe von Lupenbrillen. Die Entnahme des omentalen Lymphknotenlappens erfolgte roboterassistiert mit dem Da-Vinci-Operationsroboter.

Der in Abbildung 2 dargestellte Ablaufplan zeigt die chirurgischen Therapiemöglichkeiten an unserer Klinik, in Abhängigkeit vom Lymphknotenstatus der Patienten. 


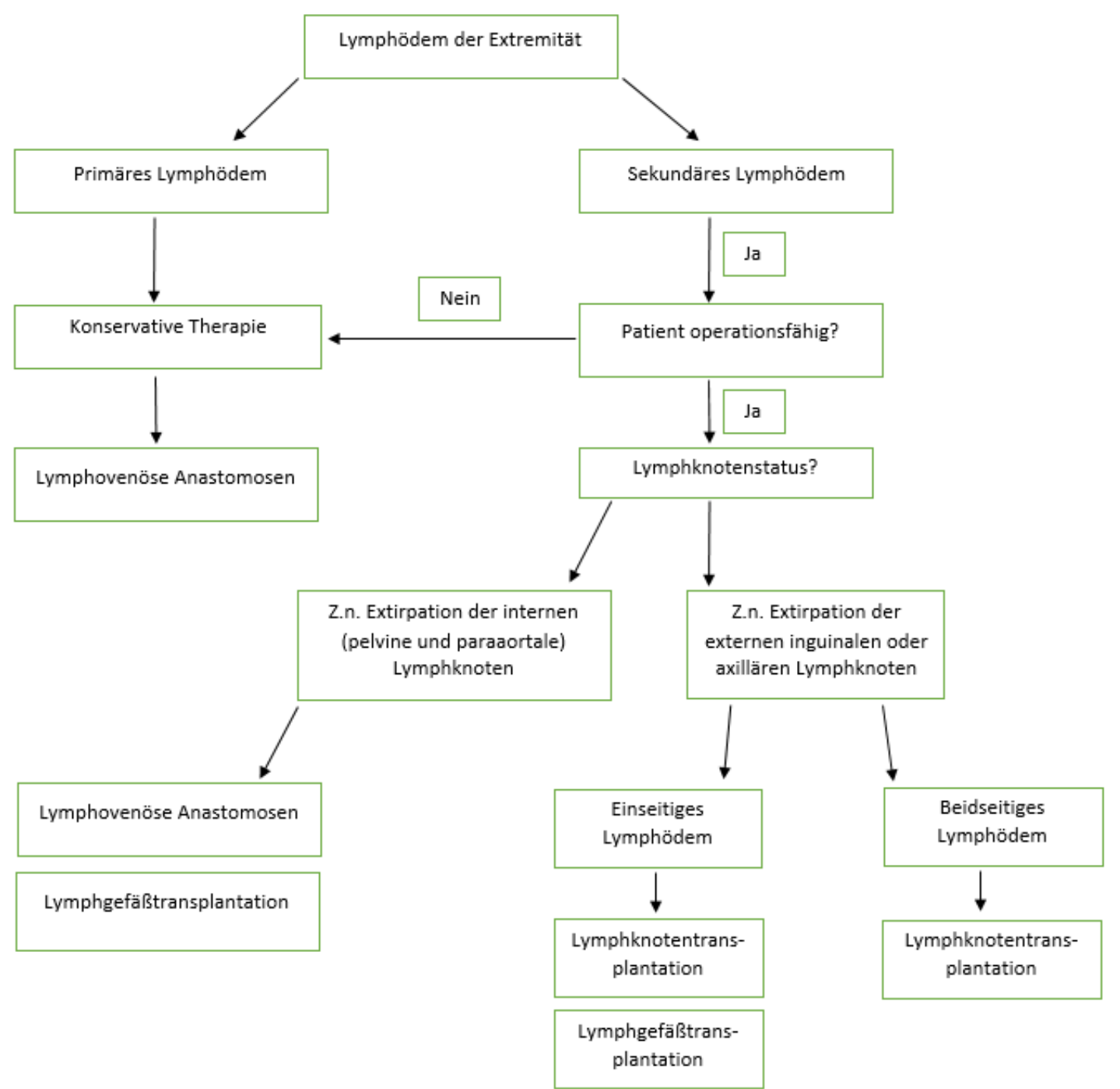

Abbildung 2: Übersichtsschema der Lymphchirurgie in der Plastischen Chirurgie in unserer Abteilung.

\subsubsection{Autologe omentale Lymphknotentransplantation roboterassistiert}

Eine absolute Notwendigkeit für die Durchführung einer omentalen roboterassistierten Lymphknotentransplantation an unserer Klinik stellt die Entfernung der inguinalen oder axillären Lymphknoten dar. Hierbei ist es wichtig, dass die Entfernung eines einzelnen Lymphknotens im Sinne eines Sentinel-Lymphknotens für die Indikationsstellung nicht ausreichend ist. Eine Transplantation in eine nichtfunktionelle Region, wie beispielsweise die Ellenbeuge oder das Sprunggelenk, wird an unserer Klinik nicht durchgeführt. Folglich werden nur Transplantationen in die Axilla oder Leiste vorgenommen.

Präoperativ erfolgt stets die gründliche körperliche Untersuchung. Aufgrund der oben genannten operativen Verfahren, wurde bei fast allen Patienten, falls nicht bei erstmaliger 
Vorstellung mitgebracht, der Operationsbericht sowie der pathologische Befundbericht der primären Operation aus der entsprechenden Klinik angefordert.

Mit Beginn der Narkose und vor dem Hautschnitt erhielt jeder Patient eine Antibiotikaprophylaxe mit $2 \mathrm{~g}$ Cefazolin und $500 \mathrm{mg}$ Metronidazol intravenös. Grundsätzlich lässt sich der Eingriff in drei Schritte einteilen. Der erste Schritt besteht in der Präparation und Darstellung der Anschlussgefäße für den omentalen Lymphknotenlappen. Der zweite Schritt beinhaltet den abdominalen Teil mit Hebung des omentalen Lappens. Im dritten Schritt wird der gehobene Lappen mit den entsprechenden Gefäßen der Leiste oder der Axilla anastomisiert. Der erste sowie auch der dritte Schritt werden unter Verwendung von Lupenbrillen durchgeführt.

Der Hautschnitt erfolgt im Regelfall unter Mitnahme des alten operativen Zugangs in Zoder S-förmiger Art und Weise. Im Bereich der Axilla haben sich die Arteria thoracodorsalis oder die Arteria thoracica lateralis mit den begleitenden Venen als zuverlässig darstellbare Anschlussgefäße gezeigt. Im Bereich der Leiste ist, besonders aufgrund der Voroperationen, eine anatomische Zuordnung oft schwieriger. Mögliche Anschlussgefäße sind hier die Arteria epigastrica superficialis, Arteria circumflexa ilium superficialis, Arteriae pudendae externae oder andere Abgänge, die die Arteria femoralis in der Leistenregion verlassen. Nach Präparation des Gefäßanschlusses, bestehend aus einer Arterie und einer Vene, erfolgt der temporäre Hautverschluss mittels Hautklammergerät sowie Folienverband.

Im zweiten Schritt wird der abdominelle, roboterassistierte Teil der Operation durchgeführt. Dies geschieht in Kooperation mit den Kollegen der Allgemein- und Viszeralchirurgie des Hauses und unter Verwendung des Da-Vinci-Xi-Robotersystems (Intuitive Surgical, Kalifornien, USA). Hierbei wird ein Videotrokar supraumbillikal sowie vier bis fünf weitere Trokare infraumbillikal eingesetzt. Die infraumbilikalen Trokare werden so gesetzt, dass sie auf den Magen und das Omentum majus ausgerichtet sind. Insgesamt werden vier Da-Vinci-Arbeitstrokare $(8 \mathrm{~mm})$ verwendet sowie ein bis zwei laparoskopische Arbeitstrokare (12 mm), über die eine laparoskopische Clipzange oder andere laparoskopische Instrumente eingeführt werden können (Abbildung 3). 

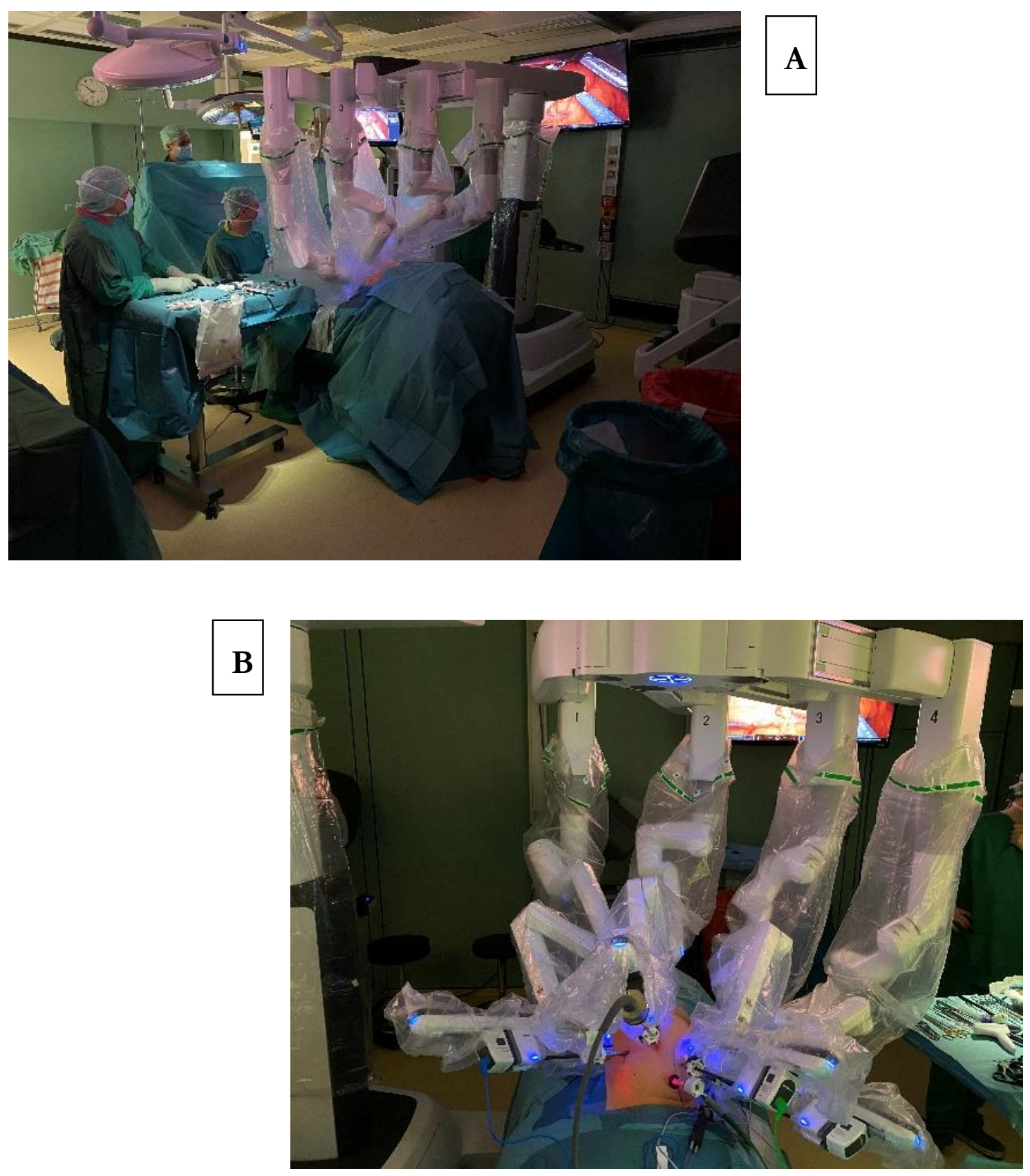

Abbildung 3: Da-Vinci-Operationsroboter in angedocktem Zustand; A Blickrichtung zur Anästhesie; B Blickrichtung auf den Patienten.

Nachdem vorhandene abdominelle Verwachsungen gelöst wurden, wird der Da-VinciRoboter angedockt. Die Präparation beginnt entlang der Arteria gastroomentalis dextra. Hierbei werden die, aus $\operatorname{der} A$. gastroomentalis dextra, zum Magen ziehenden Kollateralgefäße mit der manuellen Clipzange verschlossen und anschließend mit der bipolaren Schere des Roboters abgesetzt. Dies wird auf einer Länge von ungefähr 12-14 Zentimetern in mikrochirurgischer Präparationstechnik weitergeführt. Dann erfolgt das Absetzen des Lappens zum Omentum majus hin mit dem Vessel Sealer. Auf die zuführende Arterie sowie 
abführende Venen wird ein Gefäßclip gesetzt. Der Lappen wird mit einem Bergebeutel durch den supraumbilikalen Zugang entfernt und in eisgekühlte Bauchtücher eingeschlagen (Abbildung 4).

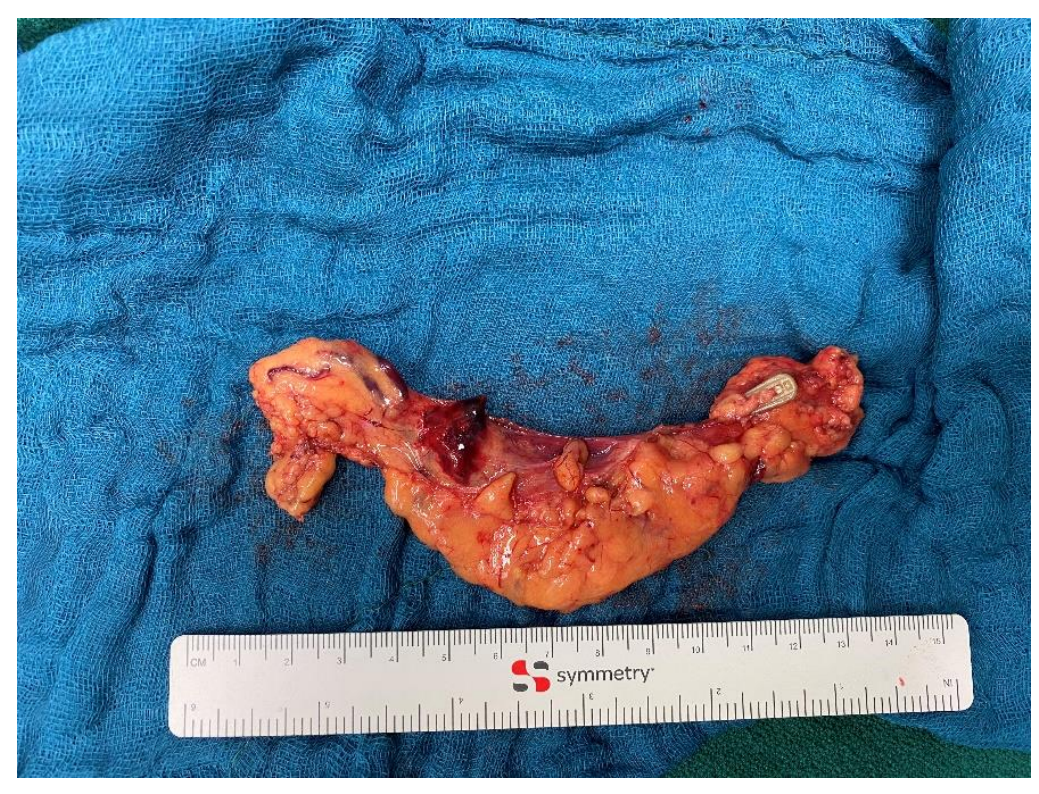

Abbildung 4: Omentaler Lymphknotenlappen nach Bergung aus dem Abdomen.

Intraabdominell wird eine Robinsondrainage eingelegt. Dann werden die Trokare entfernt und die Zugänge schichtweise verschlossen. Parallel dazu wird der Omentumlappen mit eiskalten Bauchtüchern gekühlt und zur Thromboseprophylaxe heparinisiert. Im Anschluss werden die entsprechenden Gefäße des Lappen präpariert. Dann erfolgen die Gefäßanastomosen, in der entsprechenden Empfängerregion, mit einem monofilen, nicht resorbierbaren Faden der Stärke 6-0 oder 8-0, je nach Kaliberstärke der Gefäße. Es wird eine Jackson Pratt Drainage eingelegt, der Lappen an Ort und Stelle mit resorbierbarem Nahtmaterial fixiert und die Haut schichtweise verschlossen. Zur postoperativen Überwachung hat sich ein Aufenthalt auf unserer Intermediate Care Station für eine Nacht bewährt. Eine antibiotische Abdeckung erfolgt mit Cefuroxim $500 \mathrm{mg}$ zweimal täglich für drei Wochen bis zum Fadenzug. Beide Drainagen werden abhängig von ihrer Fördermenge, jedoch frühestens nach zwei Tagen, entfernt. Eine Bewegungseinschränkung im Bereich der Armabduktion bis $90^{\circ}$, beziehungsweise Hüftflexion bis $70^{\circ}$, sollte für drei Wochen eingehalten werden. Bis zum ersten Stuhlgang wird Schonkost angeordnet. Danach kann der Kostaufbau beginnen. 


\subsection{Datenerfassung und Aufarbeitung}

Die Datenerhebung und Archivierung wurden mit dem Programm Microsoft Excel, Version 2007 (Microsoft Corporation, Washington, USA) durchgeführt.

Die statistische Auswertung, Korrelationsanalysen und graphische Darstellungen erfolgten mit dem Programm IBM SPSS Statistics Version 20 (IBM Corporation, Armonk, New York, USA) sowie Microsoft Excel, Version 2007 (Microsoft Corporation, Washington, USA). 


\section{Ergebnisse}

\subsection{Patientenkollektiv}

\subsubsection{Autologe omentale Lymphknotentransplantation roboterassistiert}

Insgesamt konnten $\mathrm{N}=18$ Patienten in die Studie eingeschlossen werden (ein Mann, 17 Frauen). Sie wurden im Zeitraum von November 2017 bis April 2020 in der Klinik für Unfallchirurgie, Orthopädie und Plastische Chirurgie der Universitätsmedizin Göttingen aufgrund eines sekundären Lymphödems behandelt. Das Durchschnittsalter der Patienten lag am Tag der operativen Versorgung bei 57,45 \pm 8,02 Jahren (Bereich: 40-75 Jahre), der mittlere Beobachtungszeitraum bei $18 \pm 3,48$ Monaten (Bereich: 12-27 Monate). Nach ISL Klassifikation wurden 15 Patienten in das Stadium II und drei Patienten in das Stadium III eingestuft. Insgesamt wurden acht Beinlymphödeme und zehn Armlymphödeme operiert. In zehn Fällen war die linke, in acht Fällen die rechte Extremität betroffen.

Das Mammakarzinom stellte mit zehn Patientinnen die häufigste primäre Diagnose dar. Die zweithäufigste Ursache war das Zervixkarzinom und das Vulvakarzinom, mit einer Häufigkeit von $N=2$. Mit einer Häufigkeit von jeweils $N=1$ erfolgte die operative Versorgung von einem Liposarkom des Oberschenkels, einem Plattenepithelkarzinom des Penis, einem malignen Melanom und einem malignen peripheren neuroektodermalen Tumor (MPNT). Sechs Patienten hatten nach primärer Operation eine Radiochemotherapie erhalten. Drei Patienten erhielten eine reine Radiatio. Zwei Patienten bekamen eine Chemotherapie. Bei allen operierten Patienten wurde im primären Eingriff eine Lymphonodektomie durchgeführt. Die mittlere Dauer bis zur operativen Versorgung betrug 91,64 Monate \pm 48,98 Monate (Bereich: 6-204 Monate). Alle Patienten profitierten, jedoch in unterschiedlicher Stärke, vom operativen Eingriff. In der Gruppe der Armlymphödeme benötigten drei der fünf Patienten in der Kontrolle nach 12 Monaten keinen Kompressionsstrumpf mehr und führten auch keine Lymphdrainage mehr durch. Eine Patientin führte ihre Lymphdrainage in der gleichen Frequenz wie präoperativ durch, konnte jedoch den Kompressionsstrumpf weglassen. Eine weitere Patientin reduzierte ihre konservative Therapie mit Kompression und manueller Lymphdrainage auf eine reine Kompression mit Strumpf und additiver Lymphdrainage einmal pro Woche in den heißen Monaten. In der Gruppe der Beinlymphödeme benötigte einer der sechs Patienten in der 
Kontrolle nach 12 Monaten keine konservative Therapie mehr. Zwei Patienten konnten auf manuelle Lymphdrainage, bei gleicher Kompression, verzichten. Eine Patientin führte die manuelle Lymphdrainage weiter und konnte ihre Kompression weglassen. Bei einem Patienten zeigte sich die Reduktion der manuellen Lymphdrainage von drei Mal wöchentlich 60 Minuten auf einmal wöchentlich 60 Minuten bei gleichbleibender Kompression. Bei einem Patienten zeigte sich nach 12 Monaten keine Änderung in der konservativen Therapie, der klinische Untersuchungsbefund imponierte jedoch deutlich weicher. Eine graphische Darstellung der Ergebnisse ist in den Abbildungen 5-7 zu sehen. Drei der operierten Patienten berichteten präoperativ über rezidivierende Erysipele der entsprechenden lymphödematösen Extremität. Zwei davon mit drei Episoden im Jahr und eine Patientin mit durchschnittlich vier Episoden. Keiner dieser Patienten erlitt im Beobachtungszeitraum erneute Erysipele der entsprechenden Extremität. Peri- sowie postoperativ kam es bei keiner der Patienten zu Komplikationen im Sinne von Wundinfektion, Wundheilungsstörungen, Nachblutungen, Hämatomen oder Seromen. Allenfalls ein leichtes Ziehen im Bereich des Abdomens wurde von drei Patienten im frühen postoperativen Verlauf angegeben. Bereits in der Kontrolle nach sechs Monaten waren keine Beschwerden mehr vorhanden.

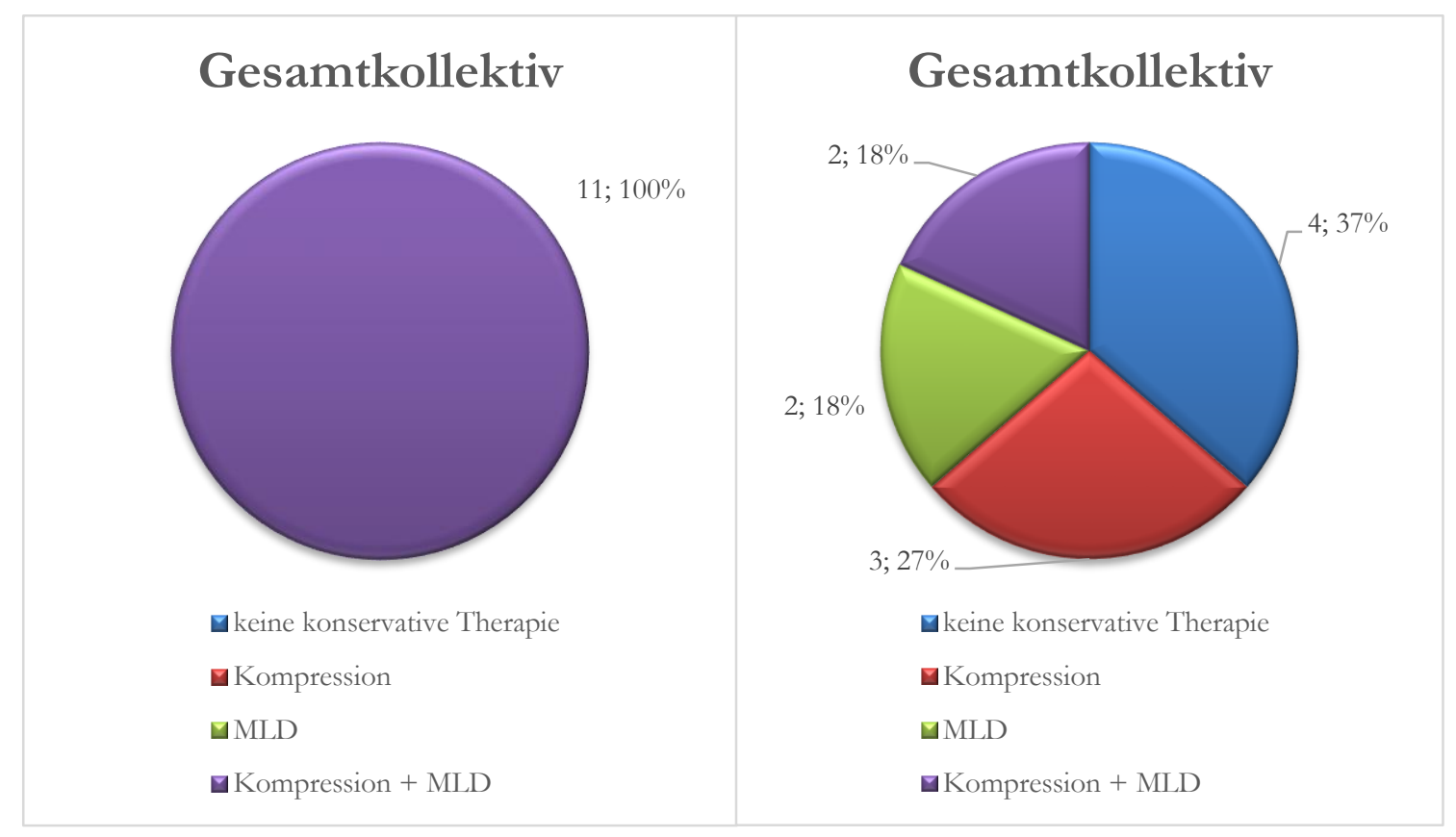

Abbildung 5: Grafische Darstellung der konservativen Therapie des Gesamtkollektives; links präoperativ, rechts postoperativ. 
Fünf der 18, in die Nachuntersuchung eingeschlossenen Patienten, wurden im Zuge der onkologischen Resektion oder anderer Erkrankungen abdominell voroperierte. Unter den durchgeführten Eingriffen waren zwei laparoskopische Hysterektomien, eine laparoskopische Ovarektomie, eine explorative Laparoskopie, eine laparoskopische Cholezystektomie sowie eine roboterassistierte Lymphonodektomie pelvin. Keine der operierten Patienten benötigte ein intraoperatives Umsteigen auf ein offenes Procedere mit medianer Laparotomie. Sowohl peri- als auch postoperativ kam es bei keinem der Patienten zu Bluttransfusionen oder Hb-relevanten Blutungen. 


\section{Armlymphödeme}

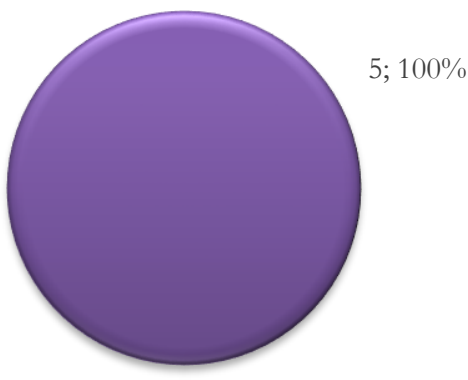

$\square$ keine konservative Therapie

- Kompression

$\square \mathrm{MLD}$

$\mathbf{\nabla}$ Kompression + MLD

\section{Armlymphödeme}

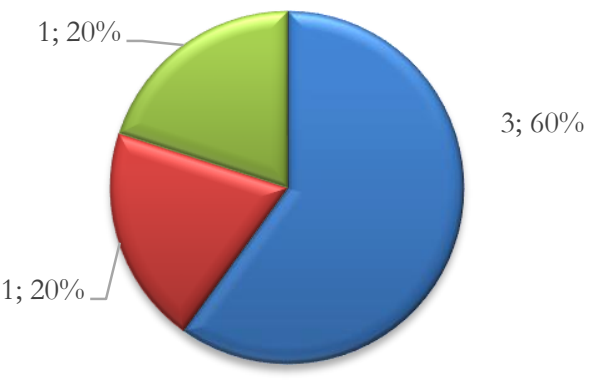

घkeine konservative Therapie

- Kompression

$\square \mathrm{MLD}$

चKompression + MLD

Abbildung 7: Grafische Darstellung der konservativen Therapie der Armlymphödeme; links präoperativ, rechts postoperativ.

\section{Beinlymphödeme}

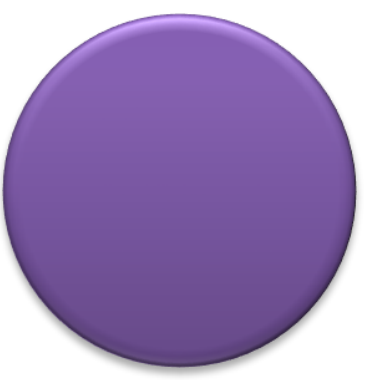

$\mathbf{v}$ keine konservative Therapie

Kompression

$\square \mathrm{MLD}$

- Kompression + MLD

\section{Beinlymphödeme}

$6 ; 100 \%$

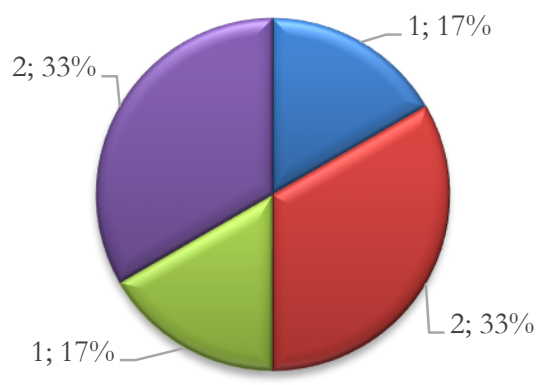

曰 keine konservative Therapie

- Kompression

$\square \mathrm{MLD}$

- Kompression + MLD

Abbildung 6: Grafische Darstellung der konservativen Therapie der Beinlymphödeme; links präoperativ, rechts postoperativ. 


\subsubsection{Autologe zervikale Lymphknotentransplantation}

Insgesamt konnten $\mathrm{N}=33$ Patienten in die Gruppe der zervikalen Lymphknoten eingeschlossen werden (zwei Männer, 31 Frauen). Sie wurden im Zeitraum von Januar 2013 bis April 2019 in der Klinik für Unfallchirurgie, Orthopädie und Plastische Chirurgie der Universitätsmedizin Göttingen aufgrund eines sekundären Lymphödems behandelt. Das Durchschnittsalter bei operativer Versorgung lag bei 49,76 \pm 4,16 Jahren (Bereich: 2277 Jahre), der mittlere Beobachtungszeitraum bei 14,15 \pm 4,9 Monaten (Bereich: 4-66 Monate). In 17 Fällen war die linke, in 16 Fällen die rechte Extremität betroffen.

Nach der Klassifikation der ISL wurden zwei Patienten in das Stadium III, die restlichen 31 in das Stadium II eingestuft. Insgesamt wurden 13 Bein- und 20 Armlymphödeme operiert. Das Mammakarzinom stellte in der zervikalen Lymphknotengruppe mit 20 Patientinnen die häufigste primäre Diagnose dar. Die zweithäufigste Ursache war das Vulvakarzinom sowie das maligne Melanom mit jeweils drei Fällen. Jeweils zwei Patientinnen wurden an einem Zervixkarzinom sowie einem Liposarkom voroperiert. Mit jeweils einem Fall wurde ein Lipom des Oberschenkels, ein Endometriumkarzinom sowie ein Lymphödem nach Leistenbruch und Entfernung der inguinalen Lymphknoten operiert.

Siebzehn Patienten hatten nach primärer Operation eine Radiochemotherapie bekommen. Eine reine Radiatio erhielten drei Patienten. Zwei Patienten bekamen eine reine Chemotherapie.

Die mittlere Dauer bis zur operativen Versorgung betrug 72,87 Monate \pm 17,36 Monate (Bereich: 6-216 Monate).

Präoperativ führten alle 33 Patienten manuelle Lymphdrainage durch und trugen Kompressionsbestrumpfung. Postoperativ konnten vier Patienten auf die komplette konservative Therapie verzichten. Neun Patienten konnten auf manuelle Lymphdrainage verzichten und trugen nur noch ihre Kompressionswäsche. Drei Patienten konnten postoperativ auf Kompressionsbestrumpfung verzichten und führten nur noch manuelle Lymphdrainage durch.

Bei den restlichen 17 Patienten zeigte sich keine Änderung im Bedarf der konservativen Therapie. Die graphische Darstellung der Veränderungen ist unter Abbildung 8-10 zu sehen.

Drei Patienten berichteten präoperativ über rezidivierende Erysipele. Bei zwei der drei Patienten trat im Nachbeobachtungszeitraum von einem Jahr kein erneutes Erysipel auf. 
Als Spenderregion wurden in unserer Klinik ausschließlich zervikale/supraklavikuläre Lymphknoten verwendet. Insgesamt wurden 24 rechte und neun linke zervikale Lymphknotenpakete entnommen und zur Transplantation verwendet.

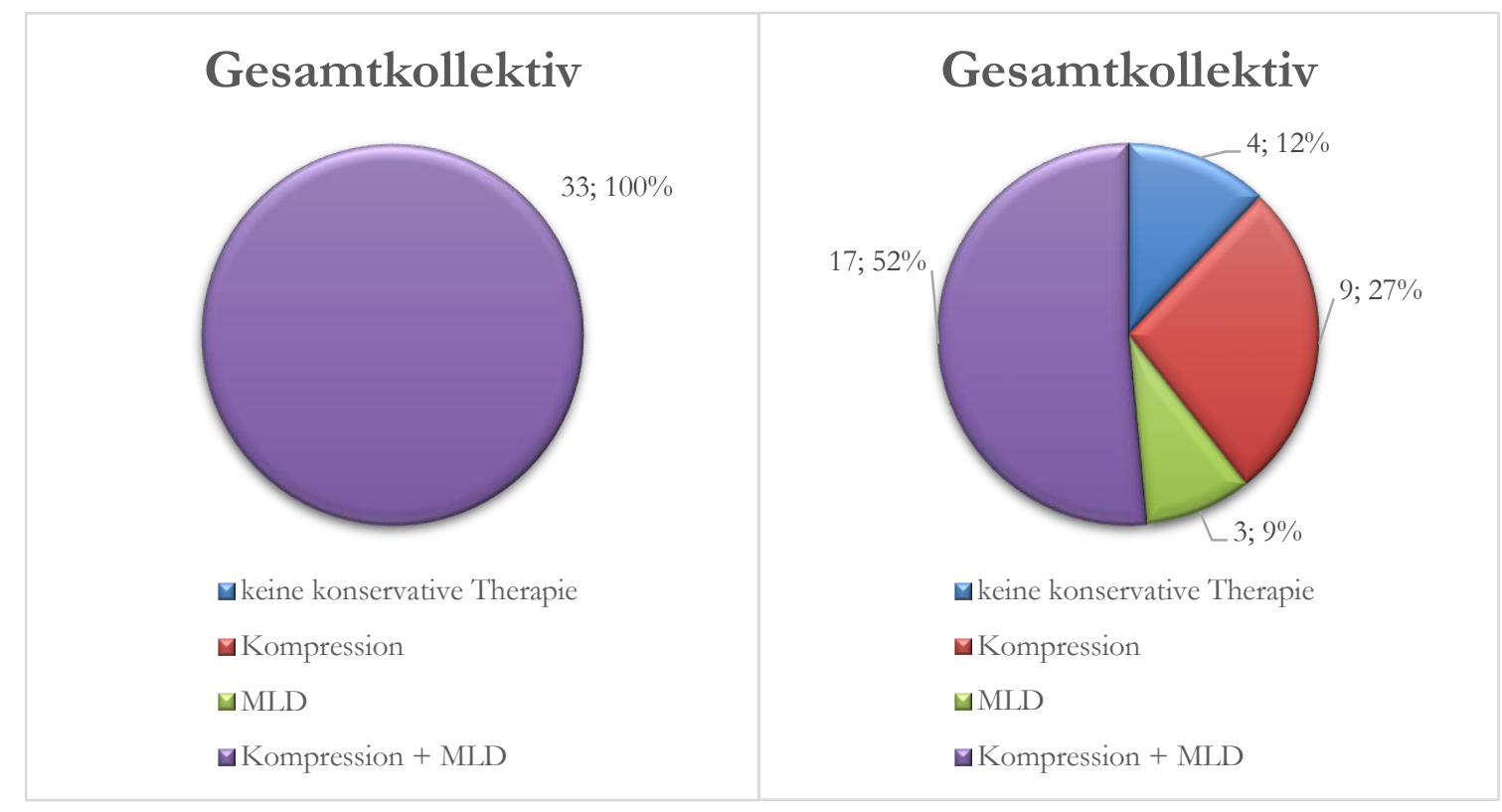

Abbildung 8: Grafische Darstellung der konservativen Therapie des zervikalen Gesamtkollektivs; links präoperativ, rechts postoperativ.

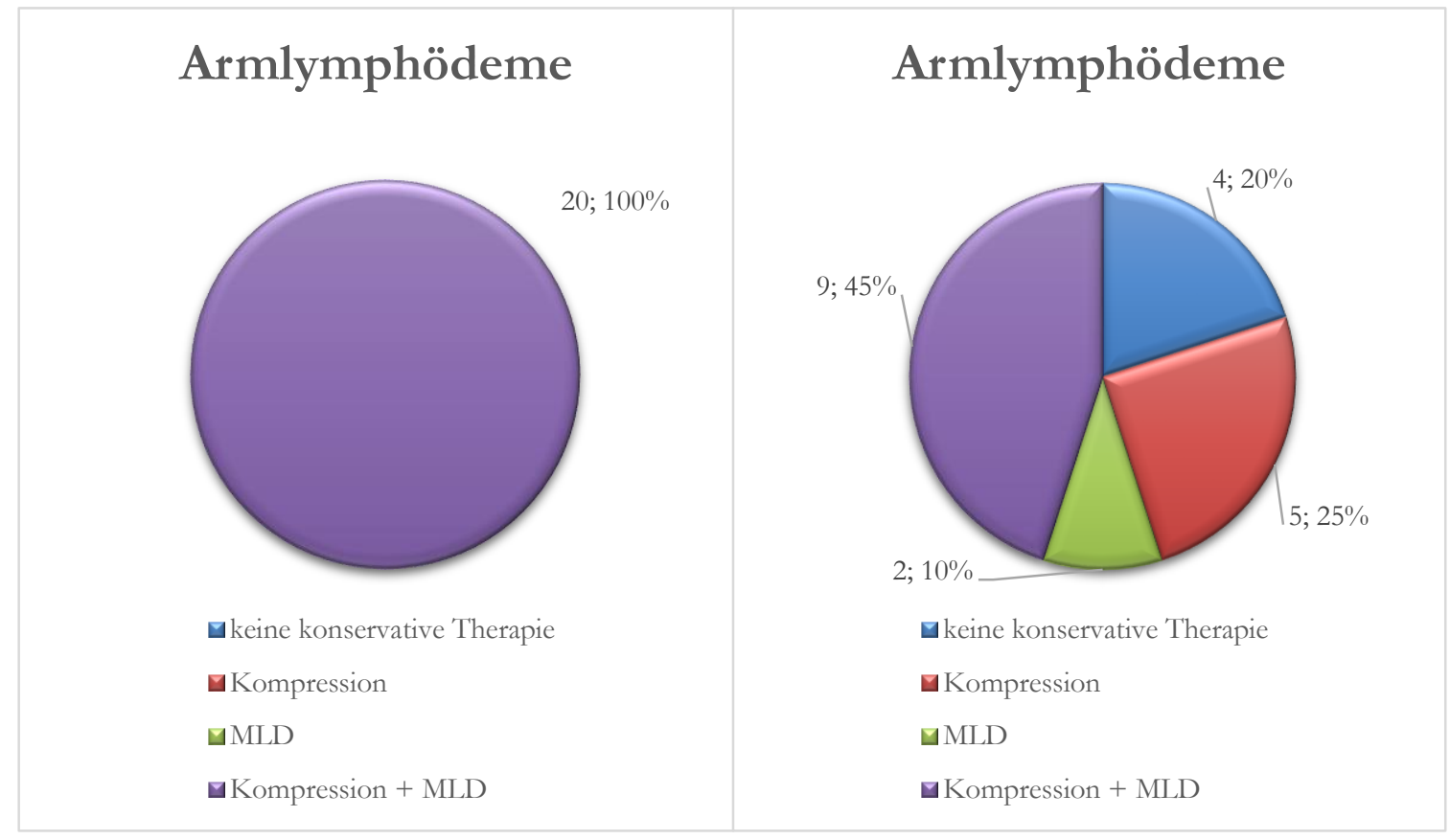

Abbildung 9: Grafische Darstellung der konservativen Therapie der zervikalen Armlymphödeme; links präoperativ, rechts postoperativ. 


\section{Beinlymphödeme}

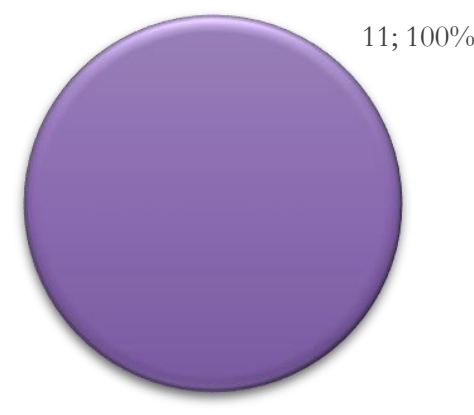

\ keine konservative Therapie

- Kompression

$\square \mathrm{MLD}$

๑Kompression + MLD

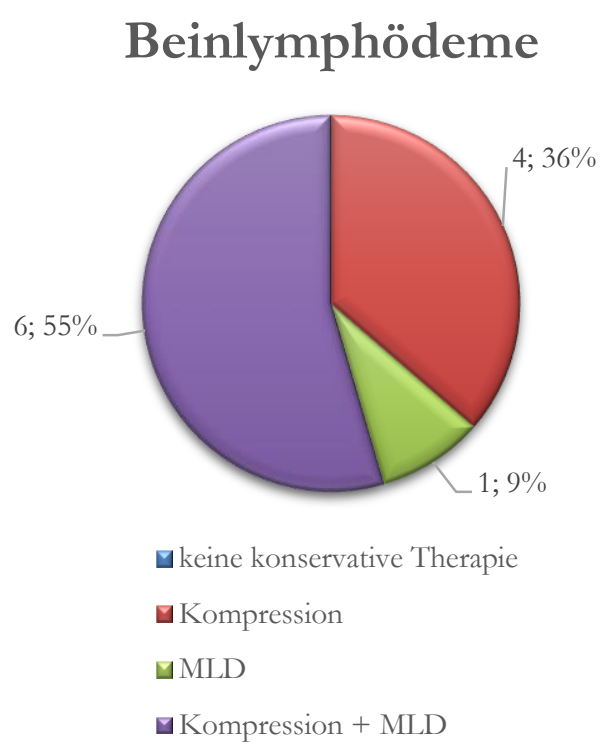

$\square$ Kompression + MLD

Abbildung 10: Grafische Darstellung der konservativen Therapie der zervikalen Beinlymphödeme; links präoperativ, rechts postoperativ.

Es traten 16 verschiedenen Komplikationen bei 33 operierten Patienten auf. Eine tabellarische Auflistung der einzelnen Komplikationen sowie deren Anzahl und Therapie findet sich in Tabelle 2. Allgemein lassen sich die Komplikationen nach Region in Hebedefektkomplikationen $(\mathrm{N}=10)$ und Komplikationen im Bereich der Empfängerstelle $(\mathrm{N}=15)$ unterteilen. Im Bereich der Spenderregion zervikal traten bei drei Patienten poststationär punktionswürdige Serome auf. Einmal zeigte sich ein Wundinfekt, der unter antibiotischer Therapie gut rückläufig war. Jeweils einmal zeigte sich stationär bzw. poststationär eine Lymphozele, welche punktiert wurden. Eine persistierende Lymphfistel im Bereich der linken Zervikalregion musste operativ revidiert und verschlossen werden. Bei deutlicher zervikaler Weichteilschwellung kam es bei einem Patienten zu einem temporären Horner Syndrom, welches im stationären Verlauf jedoch rückläufig war. In der ersten Verlaufskontrolle in unserer Sprechstunde drei Monate postoperativ war keine Symptomatik mehr vorhanden. Eine Patientin klagte postoperativ über eine hypertrophe, schmerzhafte Narbe im Bereich der Halsregion. Mit zweimaligen Triaminfiltrationen konnte hier eine deutliche Beschwerdebesserung erreicht werden. Eine weitere Patientin klagte postoperativ über eine Hyposensibilität im Bereich der Clavicula Region.

Im Bereich der Empfängerstelle kam es insgesamt sechs Mal zu Wundheilungsstörungen. In einem Fall wurde eine zusätzliche Hautnekrose im ambulanten Setting debridiert. Bei einer Patientin erfolgt die stationäre Aufnahme und antibiotische Abdeckung, nachdem sich eine Fettgewebsnekrose entleert hatte. In einem weiteren Fall kam es vier Wochen 
nach operativer Versorgung zu einem Erysipel in der operierten Region. Der entsprechende Patient hatte präoperativ nie Erysipele gehabt. In jeweils einem Fall zeigte sich ein Hämatom sowie ein Hämatoserom, welches punktiert wurde. Ein weiteres Hämatom musste offen chirurgisch revidiert werden. Eine Patientin klagte am zweiten postoperativen Tag nach Mobilisation über Atemnot. Eine durchgeführte CT Untersuchung zeigte eine Lungenarterienembolie. Nach Lysetherapie und intensivmedizinischer Überwachung war der weitere Verlauf unauffällig. Bei einem Patienten kam es poststationär zu einer Lymphfistel, welche unter eiweißreicher Kost spontan sistierte. Ein postoperatives Serom konnte erfolgreich punktiert werden. Ein beginnender Wundinfekt im stationären Verlauf konnte mit einer antibiotischen Therapie abgefangen werden. Einmalig kam es bei einer Patientin zu Beschwerden in der Leistenregion nach erfolgter Operation in selbiger Region. Die Beschwerdesymptomatik war nach 12 Monaten nicht mehr vorhanden.

Tabelle 2: Komplikationsübersicht nach zervikaler Lymphknotentransplantation.

\begin{tabular}{|c|c|c|c|}
\hline \multicolumn{4}{|c|}{ Komplikationsübersicht zervikale Lymphknotentransplantation } \\
\hline Region & Art & Anzahl & Therapie \\
\hline \multirow[t]{8}{*}{ Spenderstelle } & Horner Syndrom (stationär) & 1 & spontan rückläufig \\
\hline & Hypästhesie zervikal/claviculär (>post 1 Jahr) & 1 & \\
\hline & hypertrophe, schmerzhafte Narbe & 1 & Infiltration \\
\hline & Lymphfistel (poststationär) & 1 & operative Versorgung \\
\hline & Lymphozele (stationär) & 1 & Punktion \\
\hline & Lymphozele (poststationär) & 1 & Punktion \\
\hline & Serom (poststationär) & 3 & Punktion (mehrfach) \\
\hline & Wundinfekt (stationär) & 1 & Antibiotikum \\
\hline \multirow[t]{12}{*}{ Empfängerstelle } & Erysipel (poststationär) & 1 & Antibiotikum \\
\hline & Hämatom (poststationär) & 1 & operative Revision \\
\hline & Hämatom (poststationär) & 1 & Punktion \\
\hline & Hämatoserom (poststationär) & 1 & Punktion \\
\hline & Lungenarterienembolie (stationär) & 1 & $\begin{array}{l}\text { Lysetherapie, Intensiv } \\
\text { Überwachung }\end{array}$ \\
\hline & Lymphfistel (poststationär) & 1 & $\begin{array}{l}\text { spontan, } \\
\text { Albuminsubstitution }\end{array}$ \\
\hline & Schmerzen Leistenregion (poststationär) & 1 & spontan rückläufig \\
\hline & Serom (poststationär) & 1 & Punktion \\
\hline & Wundinfekt (stationär) & 1 & Antibiotikum \\
\hline & Wundheilungsstörung (poststationär) & 4 & $\begin{array}{l}\text { spontan, } 1 x \text { stationäre } \\
\text { Aufnahme }\end{array}$ \\
\hline & WHST mit Fettgewebsnekrose (poststationär) & 1 & $\begin{array}{l}\text { spontan, stationäre } \\
\text { Überwachung }\end{array}$ \\
\hline & WHST mit Hautnekrose & 1 & Ambulantes Debridment \\
\hline
\end{tabular}

\subsubsection{Lymphgefäßtransplantation}

In die Auswertung konnten N = 36 Patienten inkludiert werden (acht Männer, 28 Frauen). Die Behandlung beziehungsweise operative Versorgung erfolgte im Zeitraum von März 
2010 bis September 2019 in der Klinik für Unfallchirurgie, Orthopädie und Plastische Chirurgie der Universitätsmedizin Göttingen aufgrund eines sekundären Lymphödems. Das Durchschnittsalter bei operativer Versorgung lag bei 49,75 \pm 4,95 Jahren (Bereich: 15,9-60,7 Jahre), der mittlere Beobachtungszeitraum bei 14,84 \pm 4,46 Monaten (Bereich: 457 Monate). In 18 Fällen war die linke, in 15 Fällen die rechte Extremität betroffen. Drei Patienten waren beidseits betroffen.

Nach der Klassifikation der ISL wurden fünf Patienten in das Stadium III, die restlichen 31 in das Stadium II eingestuft. Insgesamt wurden 22 Bein-, 11 Arm- und zwei Lymphödeme im Bereich des Gesichts sowie ein Lymphödem im Bereich des Genitals operiert. Das Zervixkarzinom stellte, in der Lymphgefäßgruppe mit 13 Patientinnen, die häufigste primäre Diagnose dar. Die zweithäufigste Ursache war das Mammakarzinom mit elf Fällen gefolgt vom malignem Melanom mit zwei betroffenen Patienten. Des Weiteren wurde ein Endometriumkarzinom, ein Ovarialkarzinom, ein Plattenepithelkarzinom des Mundbodens, ein Prostatakarzinom, ein Leiomyosarkom, ein Seminom, ein Nierenzellkarzinom, ein Non-Hodgkin-Lymphom, ein postinfektiöses Lymphödem und ein Lymphödem nach massiver Akne vulgaris operiert.

Neunzehn Patienten hatten nach primärer Operation eine Radiochemotherapie bekommen. Eine reine Radiatio erhielten drei Patienten. Ein Patient bekam eine Chemotherapie.

Die mittlere Dauer bis zur operativen Versorgung betrug 51,45 Monate \pm 13,05 Monate (Bereich: 12-137 Monate).

Das $\mathrm{Maß}$ an konservativer Therapie war prä- und postoperativ ähnlich. Lediglich eine Patientin mit einem Armlymphödem sowie ein Patient mit Gesichtslymphödem benötigten postoperativ keine konservative Therapie mehr. Drei Patienten benötigten in den Nachkontrollen keine Lymphdrainage mehr. Die gleiche Anzahl konnte auf Kompressionsbestrumpfung verzichten. Bei den restlichen 28 Patienten trat keine Änderung im Bedarf der konservativen Therapie auf. In der Abbildung 11 erfolgt eine graphische Darstellung der Ergebnisse. 


\section{Lymphgefäßtx}

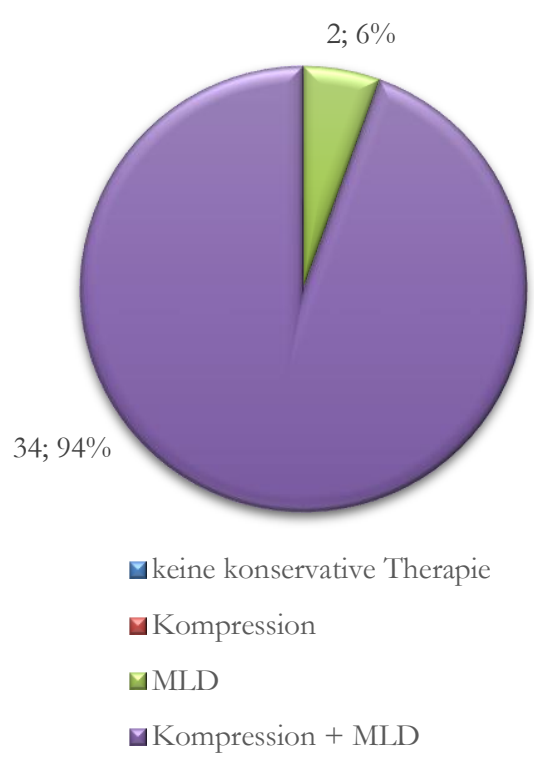

\section{Lymphgefäßtx}

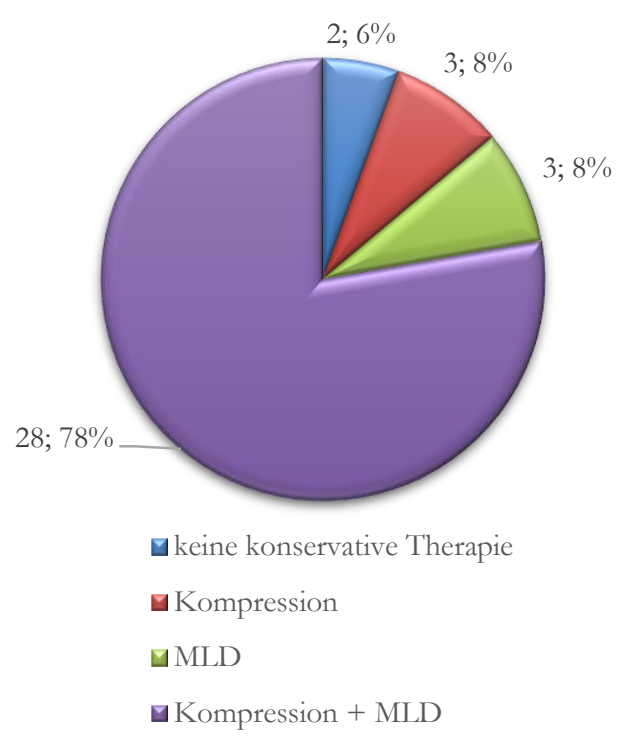

Abbildung 11: Grafische Darstellung der konservativen Therapie bei Lymphgefäßtransplantation; links präoperativ, rechts postoperativ.

Zwei Patienten berichteten präoperativ von rezidivierenden Erysipelen. Bei einem der beiden Patienten trat im Nachbeobachtungszeitraum von einem Jahr kein erneutes Erysipel auf.

Insgesamt traten neun verschiedene Komplikationen bei 36 operierten Patienten auf. Diese lassen sich unterteilen in Hebedefektkomplikationen $(\mathrm{N}=12)$ und Komplikationen am bereits geschädigten lymphödematösen Bein $(\mathrm{N}=7)$. Es traten fünf verschiedene Hebedefektmorbiditäten auf. Einmalig kam es zu einer Lymphozele, welche sich nach fünfmaliger Punktion verschloss. Zweimal waren Wunddehiszenzen mit Wundheilungsstörungen zu sehen. Zweimal kam es zu Wundinfekten, die mit entsprechender Antibiotikagabe konservativ behandelt wurden. Zweimal kam es zu Lymphfisteln, die spontan und unter Anheben der Albuminwerte sistierten. Bei fünf der 36 Patienten kam es zu einer Umfangszunahme am Spenderbein. Alle fünf Patienten wurden mit einer entsprechenden Kompressionsbestrumpfung ausgestattet. Ein Patient wurde mit einer Kompression am Fuß in Rundstrick ausgestattet. Drei Patienten benötigten Kniebestrumpfung in Rundstrick. Der letzte Patient benötigte einen kompletten Oberschenkelstrumpf in Flachstrick Kompressionsklasse I. 
Auf der Empfängerseite kam es bei drei Patienten postoperativ zu Erysipelen im Zeitraum von vier Monaten. Bei zwei von vier Patienten war es jedoch bereits präoperativ mehrmals $\mathrm{zu}$ rezidivierenden Erysipelen gekommen. Einmalig zeigte sich ein Wundinfekt mit Hautnekrose. Ein Patient berichtete über ziehende Schmerzen im Bereich der lateralen Thoraxwand mit fraglichem Zusammenhang zur operativen Versorgung. Eine Übersicht aller Komplikationen findet sich in Tabelle 3.

Tabelle 3: Komplikationsübersicht nach Lymphgefäßtransplantation.

\begin{tabular}{lllll}
\hline Komplikationsübersicht Lymphgefäßtransplantation & & \\
\hline Spenderstelle & Lymphfistel (poststationär) & 2 & $\begin{array}{l}\text { spontan, } \\
\text { Albuminsubstitution }\end{array}$ \\
& Lymphozele (poststationär) & 1 & Punktion (mehrfach) \\
\hline & Lymphödem (poststationär) & 5 & Kompression \\
\hline & Wundheilungsstörung (poststationär) & 1 & Antibiotikum \\
\hline & $\begin{array}{l}\text { Wundheilungsstörung mit Hautnektose } \\
\text { (poststationär) }\end{array}$ & 1 & operative Revision \\
& Wundinfekt (stationär) & 1 & Antibiotikum \\
\hline & Wundinfekt (poststationär) & 1 & Antibiotikum \\
\hline Empfängerstelle & Erysipel (poststationär) & & \\
\hline & Schmerzen laterale Thoraxwand (stationär) & 1 & spontan rückläufig \\
\hline & Wundinfekt (stationär) & 1 & Antibiotikum \\
\hline & Wundheilungsstörung (poststationär) & 1 & spontan \\
\hline $\begin{array}{l}\text { Wundheilungsstörung mit Hautnekrose } \\
\text { (poststationär) }\end{array}$ & 1 & operative Revision \\
\hline
\end{tabular}

\subsection{Messungen}

Alle Umfangsmessungen sowie die Erfassung der aktuellen konservativen Therapie wurden im omentalen Patientenkollektiv präoperativ, sechs Monate und zwölf Monate nach der operativen Versorgung durchgeführt. Die Messung der Operationszeiten des Da-VinciOperationsroboters erfolgten intraoperativ. Hierbei wurde nicht nur die aktive Arbeit an der Konsol des Da-Vinci erfasst, sondern die komplette Zeit des abdominellen Eingriffs. Die Zeitmessung begann mit dem Hinzukommen des Allgemein- und Viszeralchirurgen an den Operationstisch und endete mit dessen Abtreten. 


\subsubsection{Umfangsmessung}

In der Tabelle 4 sind die Mittelwerte der Umfänge der operierten Extremitäten präoperativ, sechs sowie zwölf Monate nach durchgeführtem Eingriff aufgezeigt. In den Abbildungen 12-14 erfolgt die grafische Darstellung mit Liniendiagrammen. Abbildung 15-17 zeigen selbige Werte in Form von Box Plots.

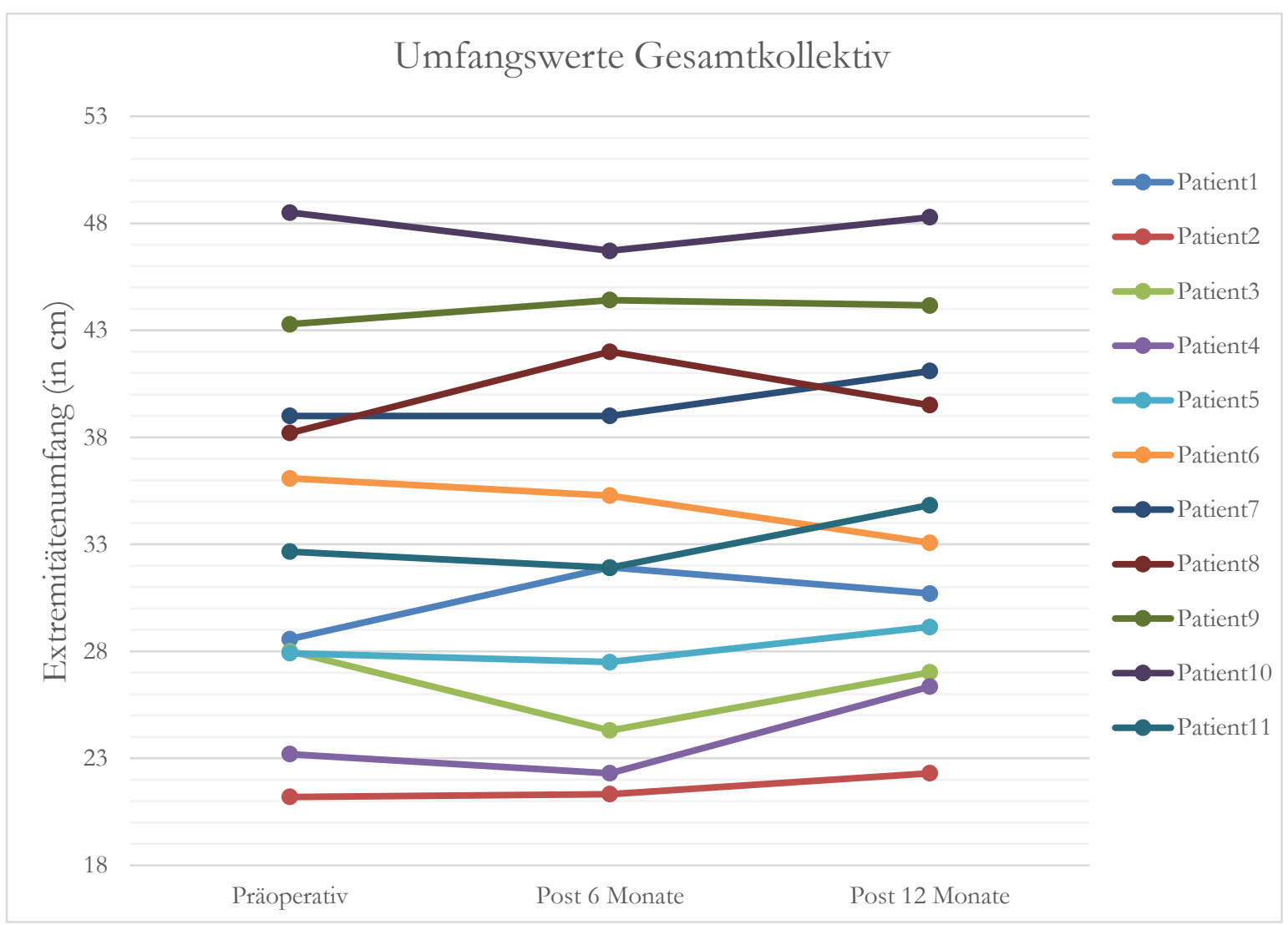

Abbildung 12: Die Abbildung zeigt die für jeden Patienten berechneten Mittelwerte der Extremitätenumfänge prä- sowie postoperativ in Zentimeter. 


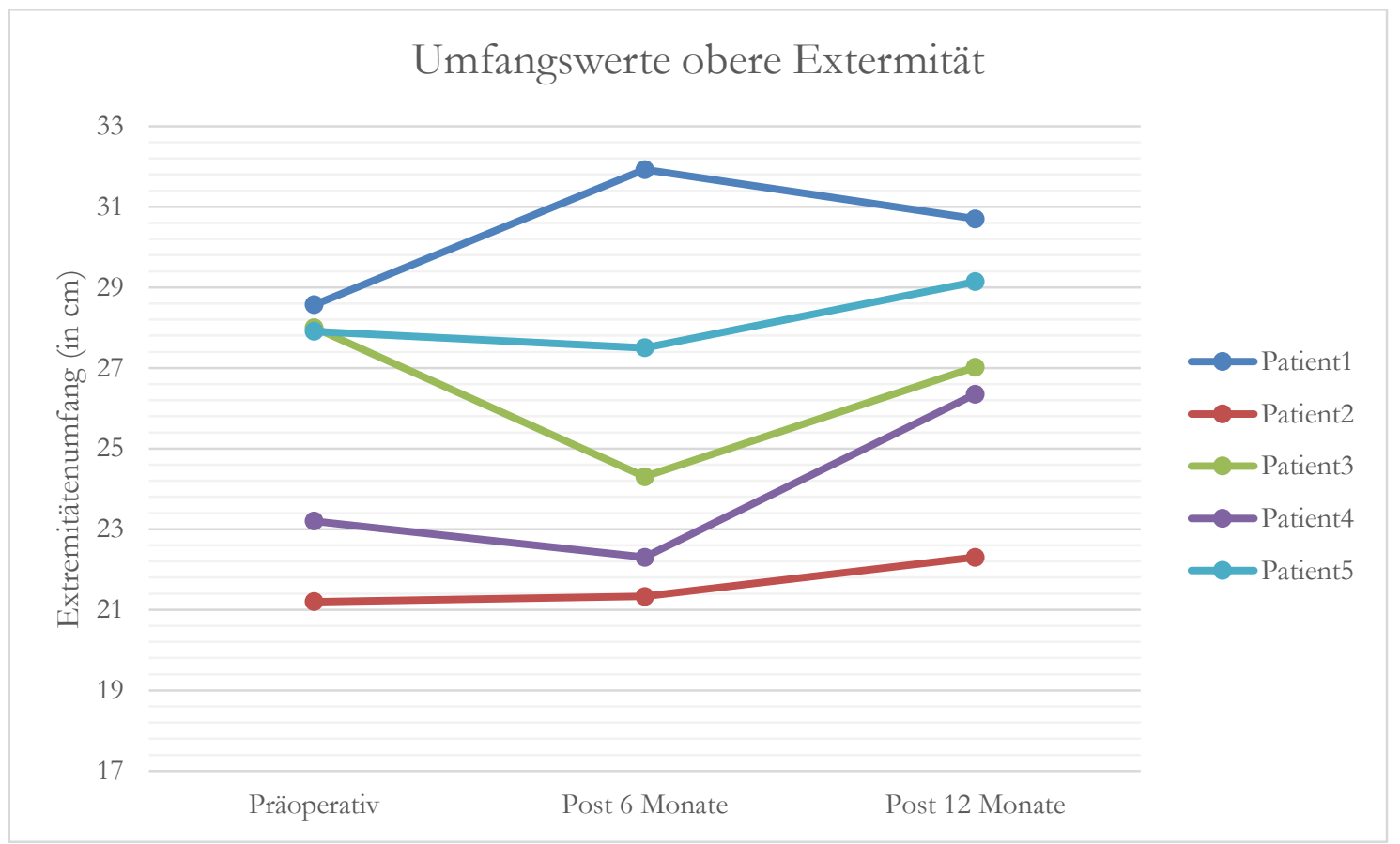

Abbildung 13: Die Abbildung zeigt die für jeden Patienten berechneten Mittelwerte der Extremitätenumfänge prä- sowie postoperativ in Zentimeter an der oberen Extremität.

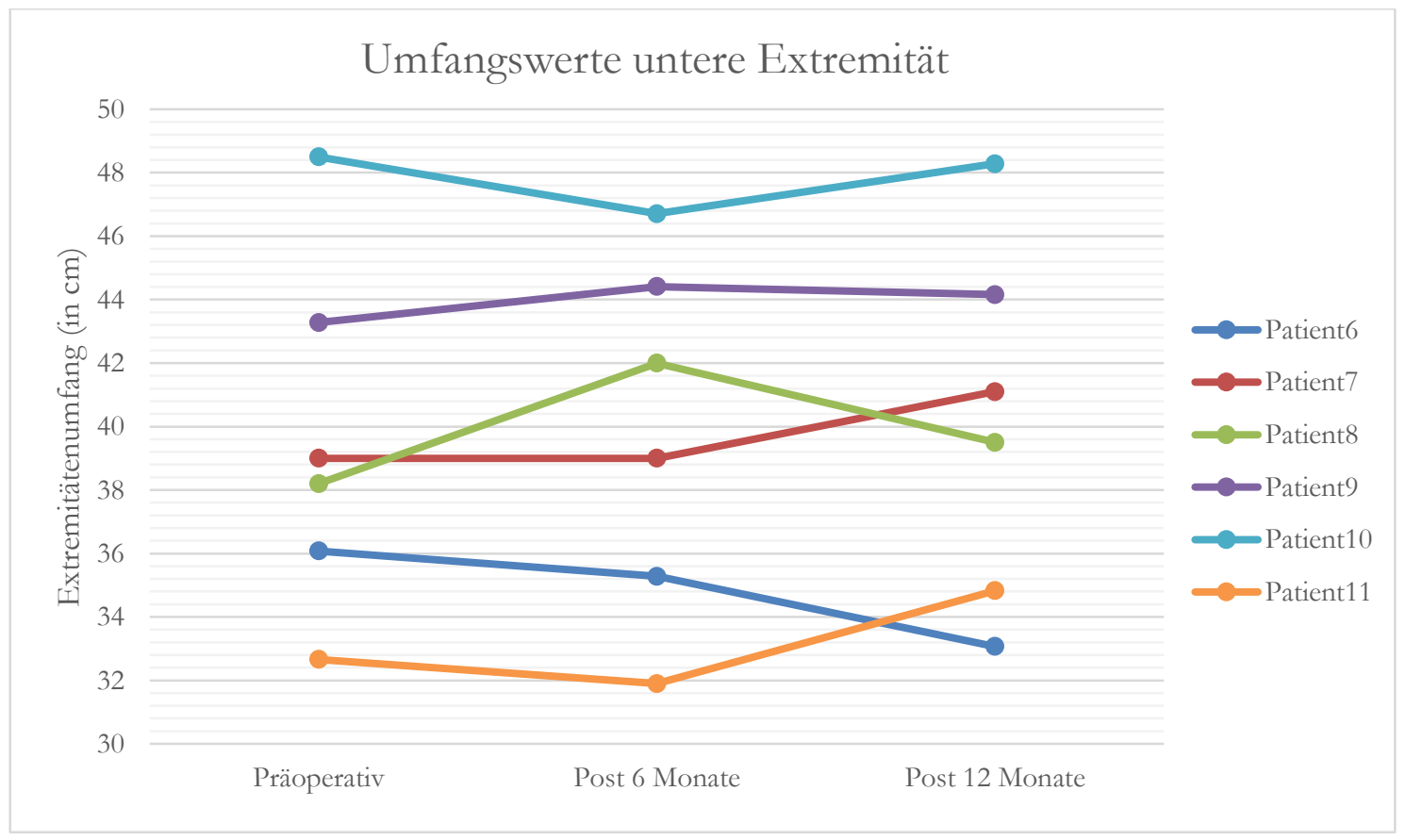

Abbildung 14: Die Abbildung zeigt die für jeden Patienten berechneten Mittelwerte der Extremitätenumfänge prä- sowie postoperativ in Zentimeter an der unteren Extremität. 
Tabelle 4: Die Tabelle zeigt die berechneten Mittelwerte $\pm 0,95$ Konfidenzintervall in Zentimetern präoperativ, nach sechs und nach 12 Monaten für das Gesamtkollektiv sowie die obere und untere Extremität.

\begin{tabular}{|l|l|l|l|}
\hline $\begin{array}{l}\text { Zeitpunkt der } \\
\text { Messung }\end{array}$ & Gesamtkollektiv & Obere Extremität & Untere Extremität \\
\hline Präoperativ & $33,32 \pm 5,71$ & $25,77 \pm 4,16$ & $41,01 \pm 6,13$ \\
\hline $\begin{array}{l}\text { 6 Monate } \\
\text { postoperativ }\end{array}$ & $33,33 \pm 6$ & $25,47 \pm 5,36$ & $41,48 \pm 5,59$ \\
\hline $\begin{array}{l}\text { 12 Monate } \\
\text { postoperativ }\end{array}$ & $34,22 \pm 5,49$ & $27,1 \pm 3,97$ & $41,22 \pm 7,02$ \\
\hline
\end{tabular}

\section{Gesamtkollektiv}

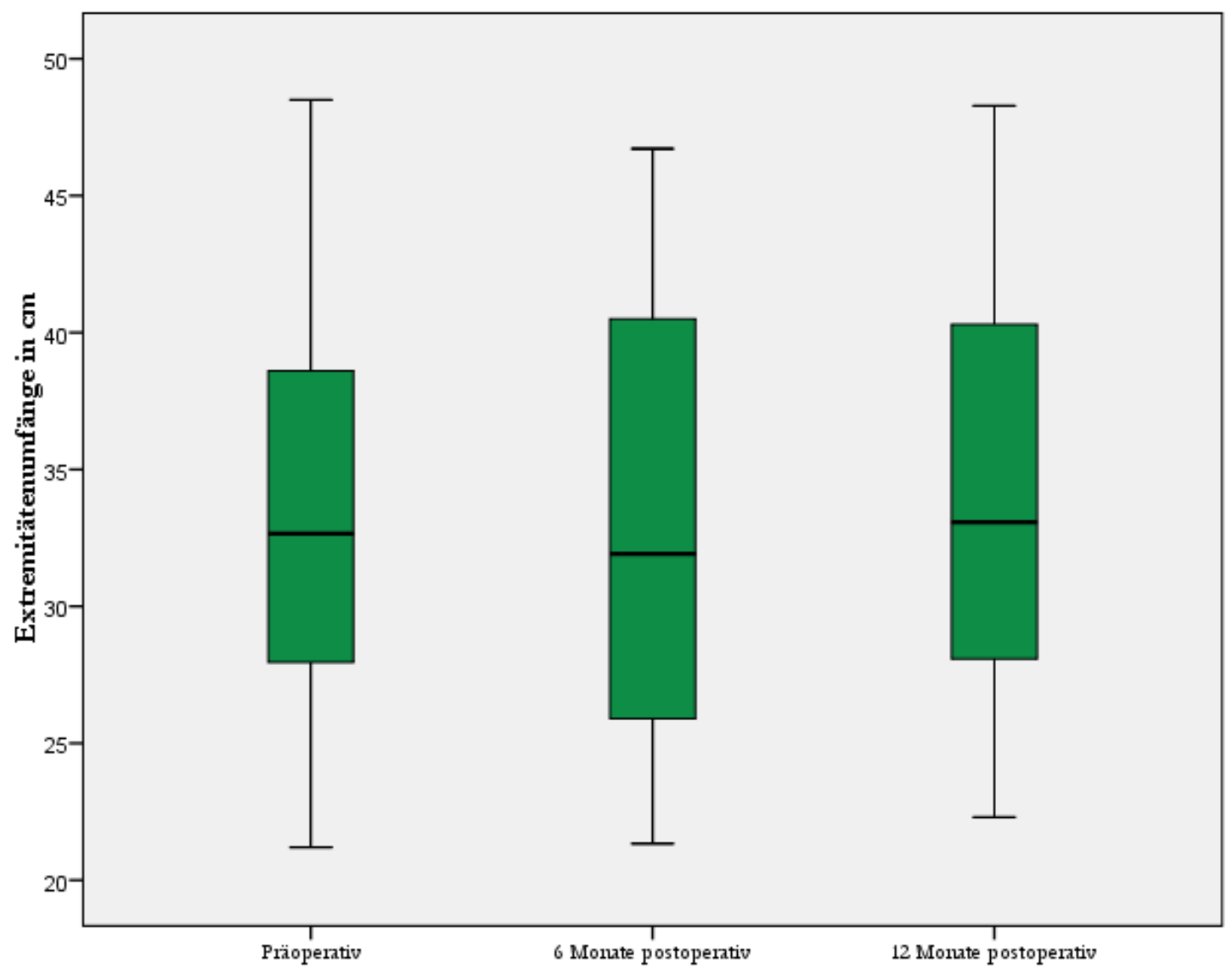

Abbildung 15: Die Box Plots zeigen die Umfangswerte in Zentimetern, Minimum, Maximum, Median und untere und obere Perzentile des Gesamtkollektivs. 
obere Extremität

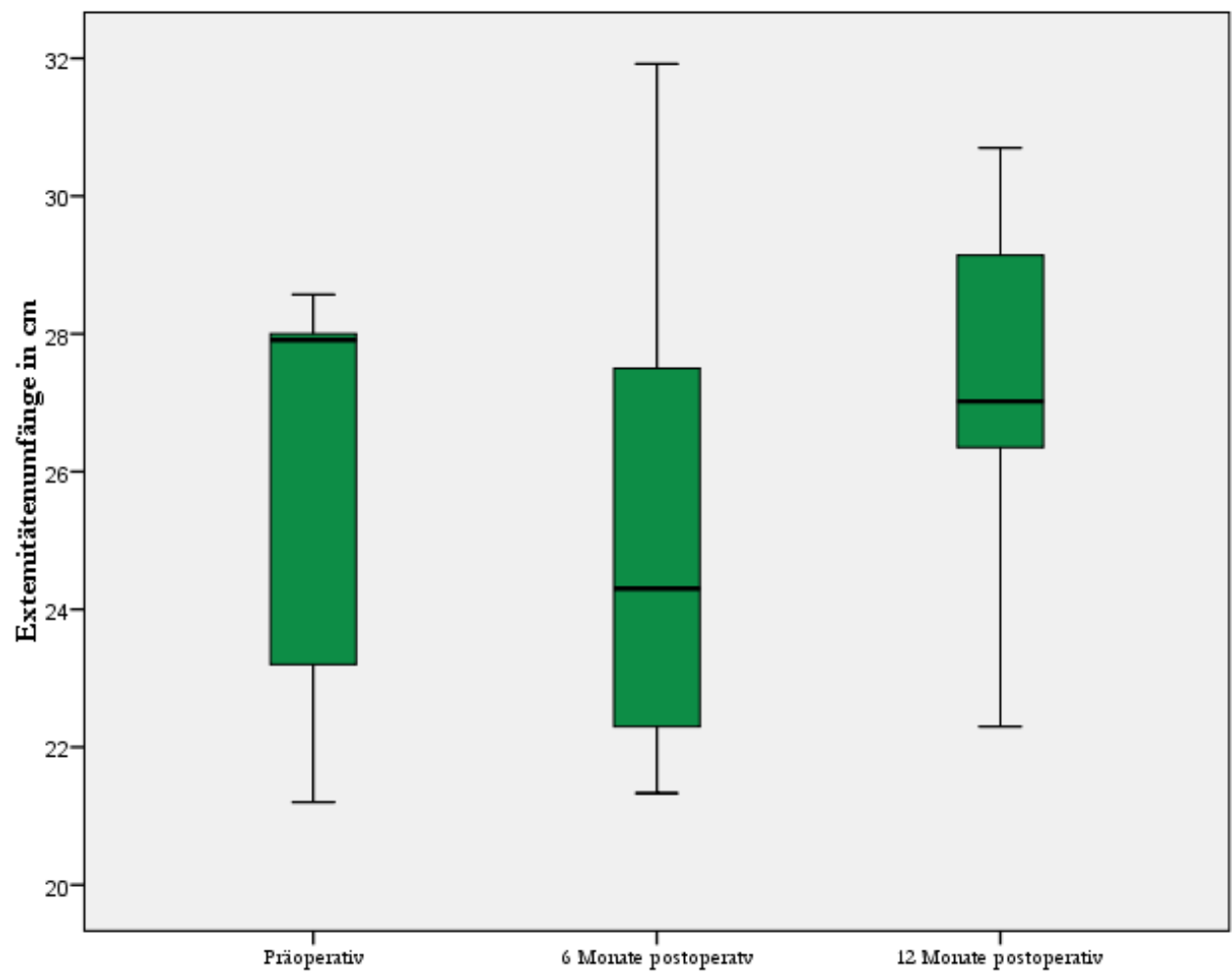

Abbildung 17: Die Box Plots zeigen die Umfangswerte in Zentimetern, Minimum, Maximum, Median und untere und obere Perzentile der oberen Extremitäten.

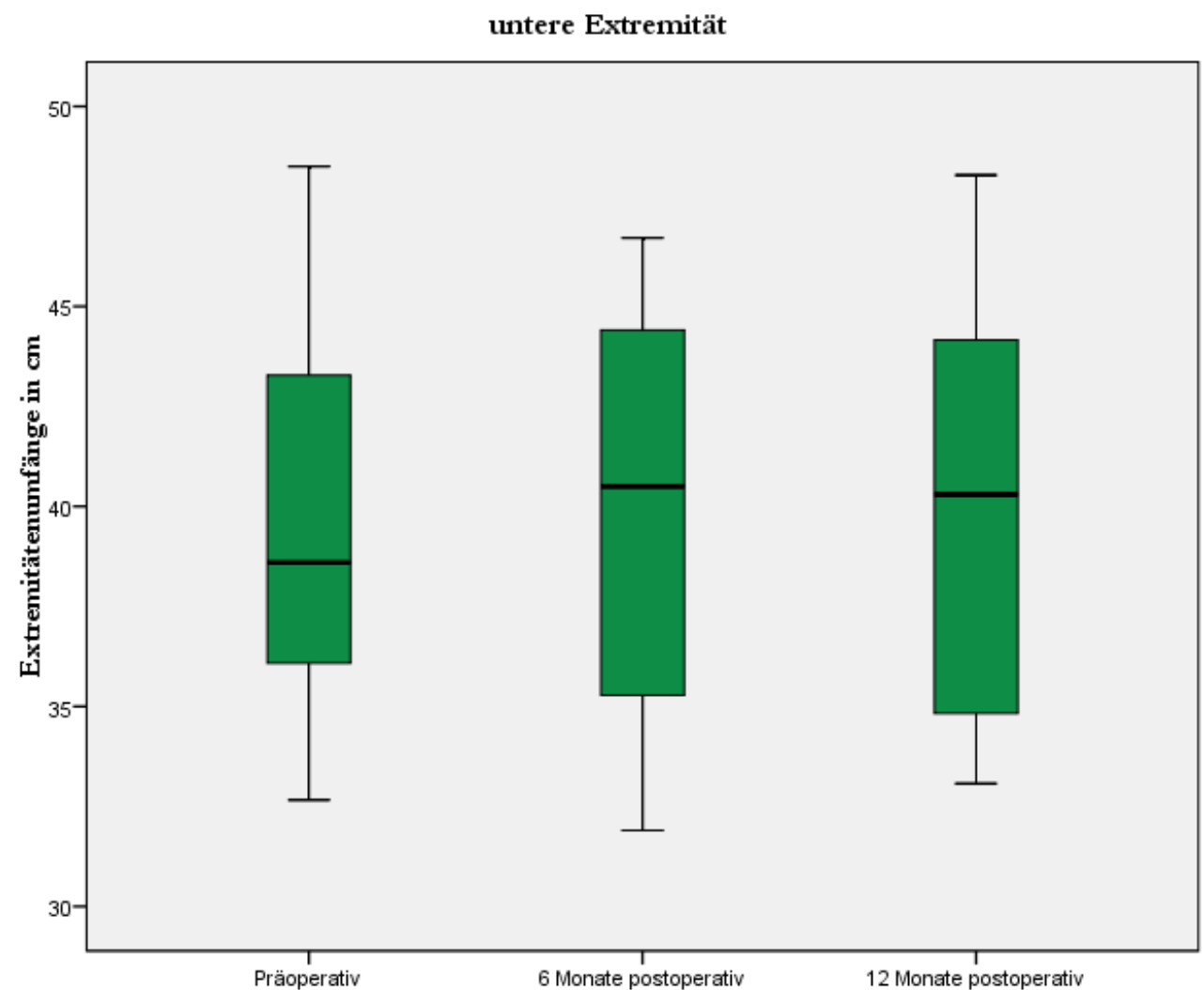

Abbildung 16: Die Box Plots zeigen die Umfangswerte in Zentimetern, Minimum, Maximum, Median und untere und obere Perzentile der unteren Extremitäten. 


\subsubsection{Konservative Therapie}

Bei unserem Gesamtkollektiv von 80 operierten Patienten konnten zehn Patienten (12,5\%) postoperativ (Nachbeobachtungszeitraum: Omentale LK $=18 \pm 3,48$ Monaten; Zervikale $\mathrm{LK}=14,15 \pm$ 4,9 Monaten; Lymphgefäßtx $=14,84 \pm$ 4,46 Monaten) auf die komplette konservative Therapie, bei klinisch stabilem Lymphödem, verzichten. Rechnerisch und in Relation zum jeweiligen Gesamtkollektiv ergeben sich somit für die omentalen Lymphknoten $=36,36 \%$; Zervikalen Lymphknoten $=12,12 \%$ und für die Lymphgefäße $=$ $5,55 \%$.

Im Gesamtkollektiv konnten $10 \%$ der operierten Patienten auf eine Kompressionsbestrumpfung komplett verzichten (Omentale LK $=18,18 \%$; Zervikale LK $=9,09 \% ;$ Lymphgefäßtx $=8,33 \%$ ). Eine reine Kompression ohne manuelle Lymphdrainage führten 18,75\% (Omentale $\mathrm{LK}=27,27 \%$; Zervikale $\mathrm{LK}=27,27 \%$; Lymphgefäßtx $=8,33 \%$ ) durch. Eine auflistende Darstellung erfolgt in Tabelle 5 .

Tabelle 5: Übersicht der konservativen Therapien im Vergleich (insgesamt sowie aufgeteilt nach entsprechendem Eingriff).

\begin{tabular}{|l|r|r|r|r|}
\hline & Gesamteingriffe & Omentale LK & Cervikale LK & Lymphgefäßtx \\
\hline Therapie Insgesamt & 80 & 11 & 33 & 36 \\
\hline $\begin{array}{l}\text { keine konservative } \\
\text { Therapie }\end{array}$ & $10 ;(12,5 \%)$ & $4 ;(36,36 \%)$ & $4 ;(12,12 \%)$ & $2 ;(5,55 \%)$ \\
\hline Nur MLD & $8 ;(10 \%)$ & $2 ;(18,18 \%)$ & $3 ;(9,09 \%)$ & $3 ;(8,33 \%)$ \\
\hline Nur Kompression & $15 ;(18,75 \%)$ & $3 ;(27,27 \%)$ & $9 ;(27,27 \%)$ & $3 ;(8,33 \%)$ \\
\hline MLD + Kompression & $47 ;(58,75 \%)$ & $2 ;(18,18 \%)$ & $17 ;(51,51 \%)$ & $28 ;(77,77 \%)$ \\
\hline
\end{tabular}

\subsubsection{Operationszeiten}

3.2.3.1 Autologe omentale Lymphknotentransplantation roboterassistiert

Insgesamt wurden 18 operative Eingriffe in der Klinik für Unfallchirurgie, Orthopädie und Plastische Chirurgie in die Auswertung eingeschlossen. Der Erhebungszeitraum erstreckte sich von November 2017 bis Januar 2020. Die Messung der Gesamtoperationszeit begann mit dem Hautschnitt und endete mit der Hautnaht. Die Da-Vinci-Zeitmessung begann mit der Übernahme des Operationsgebietes durch den Allgemein- und Viszeralchirurgen und endete mit dessen Abtreten nach vollendeter Hautnaht im Bereich des Abdomens.

Der Mittelwert aller operativen Eingriffe belief sich auf 312,77 Minuten \pm 16,66 Minuten mit einem Median von 322,00 Minuten. Die kürzeste Gesamtoperationszeit betrug 219 
Minuten. Die längste Eingriffszeit lag bei 350 Minuten. Somit ergab sich eine Spannweite von 131 Minuten für die operativen Eingriffe.

Der Einsatz des Da-Vinci-Operationsroboters, inklusive vorbereitenden Maßnahmen, benötigte durchschnittlich 123,3 Minuten \pm 16,77 Minuten mit einem Median von 130 Minuten. Die kürzeste Operationszeit in diesem Abschnitt waren 73 Minuten. Die längste lag bei 190 Minuten mit einer Spannweite von 117 Minuten.

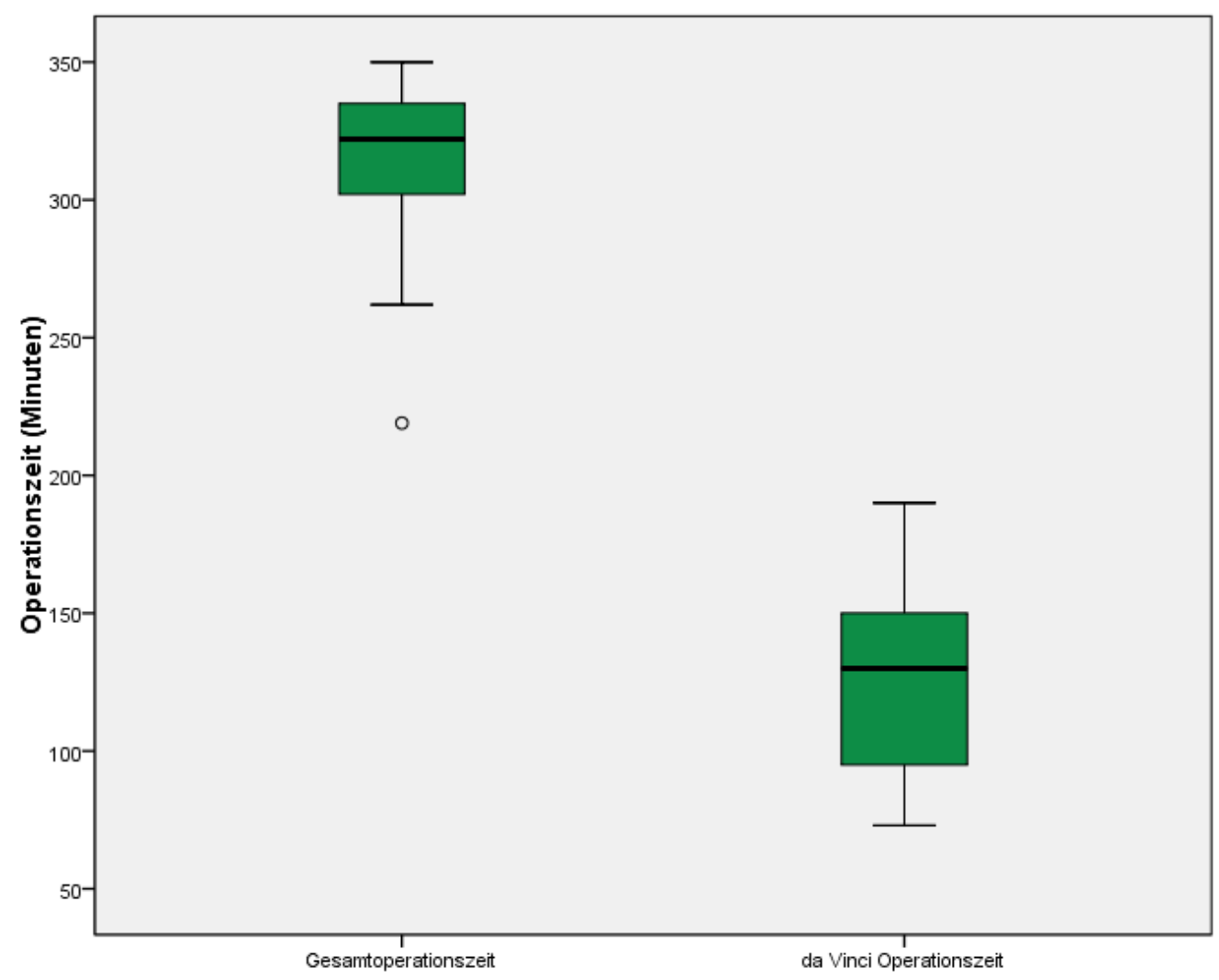

Abbildung 18: Operationszeiten in Minuten; links: Gesamtoperationszeit aller erfassten Eingriffe, rechts: Operationszeit mit dem Da-Vinci-Roboter jeweils mit Minimum, Maximum, Konfidenzintervall, Median und entsprechenden Ausreißern. 
3.2.3.2 Autologe zervikale Lymphknotentransplantation

Insgesamt wurden 33 operative Eingriffe in der Klinik für Unfallchirurgie, Orthopädie und Plastische Chirurgie in die Auswertung der zervikalen Lymphknotentransplantation eingeschlossen. Der Erhebungszeitraum erstreckte sich von Januar 2013 bis Februar 2019. Die Messung der Gesamtoperationszeit begann mit dem Hautschnitt und endete mit der Hautnaht. Der Mittelwert aller operativen Eingriffe belief sich auf 340,66 Minuten $\pm 19,8$ Minuten mit einem Median von 322 Minuten. Die kürzeste Gesamtoperationszeit waren 185 Minuten. Die längste Eingriffszeit lag bei 592 Minuten. Somit ergab sich eine Spannweite von 407 Minuten für die operativen Eingriffe.

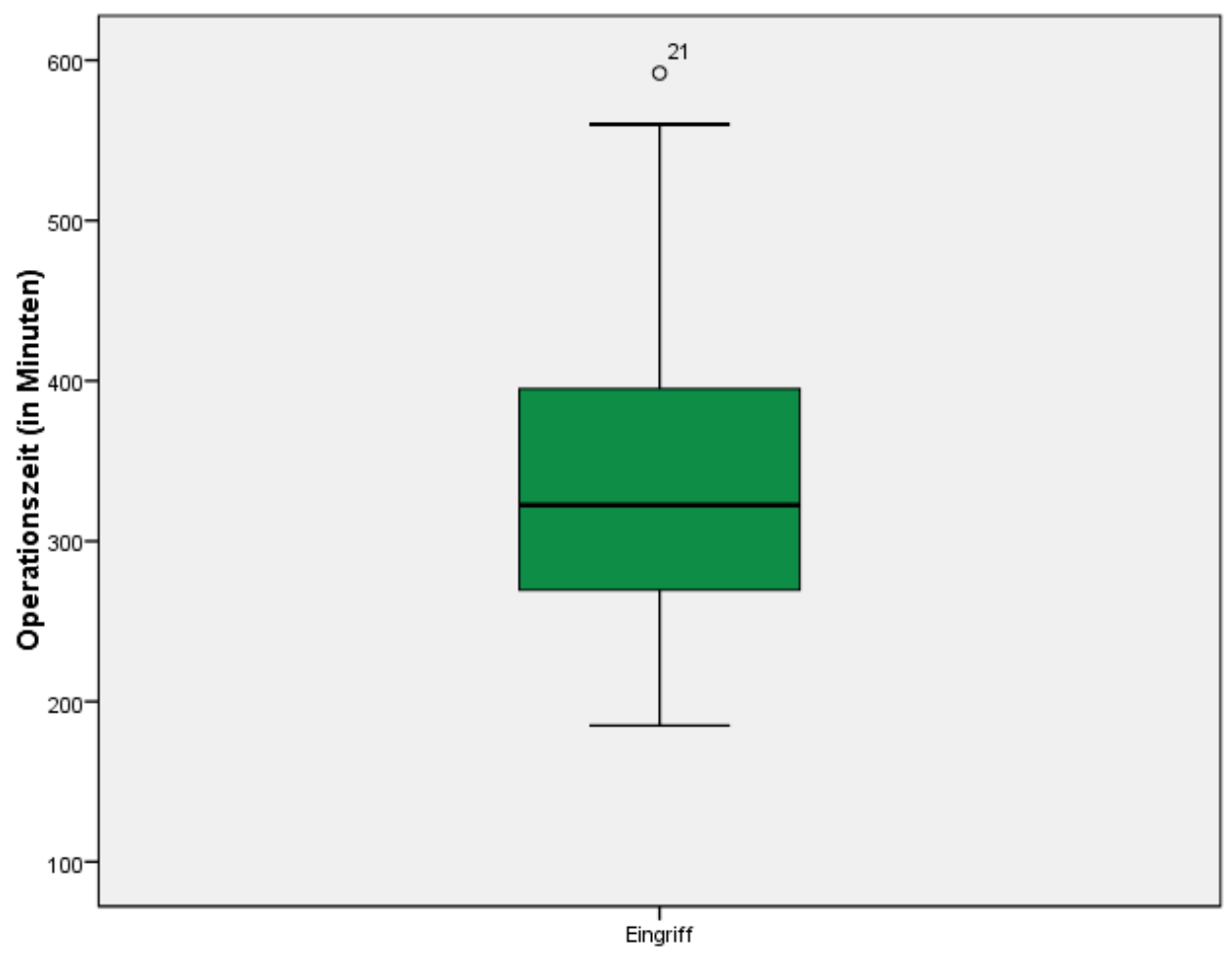

Abbildung 19: Operationszeit der zervikalen Lymphknotentransplantation mit Minimum, Maximum, Konfidenzintervall, Median und entsprechenden Ausreißern. 


\subsubsection{Lymphgefäßtransplantation}

Insgesamt wurden 36 operative Eingriffe in der Klinik für Unfallchirurgie, Orthopädie und Plastische Chirurgie, in die Auswertung der Lymphgefäßtransplantation, eingeschlossen. Der Erhebungszeitraum erstreckte sich von März 2010 bis Juni 2018. Die Messung der Gesamtoperationszeit begann mit dem Hautschnitt und endete mit der Hautnaht. Der Mittelwert aller operativen Eingriffe belief sich auf 278,60 Minuten \pm 19,8 Minuten mit einem Median von 271 Minuten. Die kürzeste Gesamtoperationszeit lag bei 180 Minuten. Die längste Eingriffszeit lag bei 465 Minuten. Somit ergab sich eine Spannweite von 285 Minuten für die operativen Eingriffe.

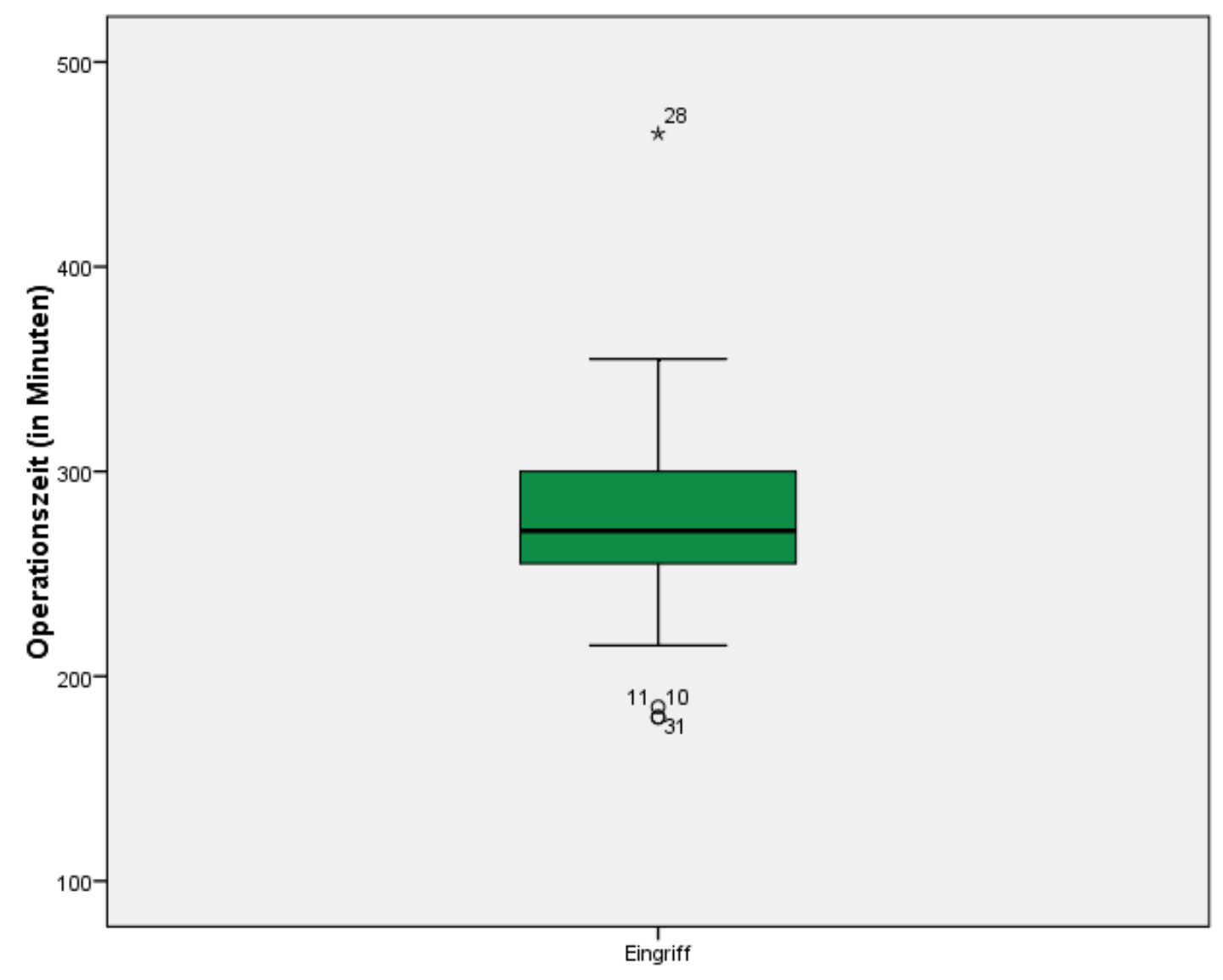

Abbildung 20: Operationszeit der Lymphgefäßtransplantationen mit Minimum, Maximum, Konfidenzintervall, Median und entsprechenden Ausreißern. 


\subsection{Korrelationsanalyse}

\subsubsection{Umfangsmessung}

Um einen möglichen Zusammenhang zwischen der durchgeführten omentalen Lymphknotentransplantation und dem Umfang der betroffenen Extremitäten darzustellen, wurde eine Varianzanalyse durchgeführt. Bei abhängigen Stichproben mit mehr als zwei Gruppen und Verteilungsfreiheit wurde der Friedmann Test angewendet. Ein p-Wert von $\mathrm{p}<0,05$ wurde als signifikant gewertet. Es wurden sowohl die Mittelwerte der Gesamtumfänge als auch die Mittelwerte für die obere und untere Extremität getrennt berechnet. Es konnte keine Korrelation zwischen dem chirurgischen Eingriff und den präoperativen und postoperativen Umfangswerten gezeigt werden.

Abbildungen 12-17 und Tabelle 4 zeigen die Veränderung der Extremitätenumfänge aller im Zeitraum operierten Patienten. In Tabelle 4 werden jeweils die Mittelwerte $\pm 0,95$ Konfidenzintervall (KI) aufgezeigt. In den Box Plots erfolgt die Darstellung der entsprechenden deskriptiven Statistik. Bezüglich der mittleren postoperativen Umfänge kam es in unserem Patientenkollektiv zu keiner klar darstellbaren Veränderung. Dies lässt sich anhand der Einzelfallbetrachtung aus Abbildung 12 erklären. Hier sind Liniendiagramme mit negativer sowie auch positiver Tendenz zu erkennen. Daraus resultiert, bei Gesamtbetrachtung aller erhobenen Umfänge, keine signifikante Veränderung in den Messwerten der Umfänge.

\subsubsection{Operationszeiten}

\subsubsection{Autologe omentale Lymphknotentransplantation roboterassistiert}

Für die Korrelationsuntersuchung wurde zuerst die Normalverteilung der Messwerte getestet. Sowohl im grafischen Histogramm, im Kolmogorov-Smirnov, als auch im Shapiro-Wilk Test für Normalverteilung kann keine Normalverteilung nachgewiesen werden.

Tabelle 6: Testung auf Normalverteilung des omentalen Patientenkollektivs.

Tests auf Normalverteilung

\begin{tabular}{|l|r|r|r|r|r|r|}
\hline & \multicolumn{3}{|c|}{ Kolmogorov-Smirnov } & \multicolumn{3}{c|}{ Shapiro-Wilk } \\
\cline { 2 - 7 } & Statistik & \multicolumn{1}{c|}{ Df } & Signifikanz & Statistik & \multicolumn{1}{c|}{ Df } & Signifikanz \\
\hline Zeit_daVinci &, 189 & 18 &, 087 &, 944 & 18 &, 343 \\
Zeit_Gesamt &, 193 & 18 &, 075 &, 845 & 18 &, 007 \\
\hline
\end{tabular}


Somit erfolgt die Korrelationsanalyse im Folgenden (Tabelle 7 und 8) mit der

Rangkorrelation nach Spearman für abhängige, nicht normalverteilte Variablen.

Tabelle 7: Korrelationsanalyse der Gesamtoperationszeiten mit der Operationsanzahl für die omentalen Lymphknotentransplantationen.

Korrelationen

\begin{tabular}{|cll|r|r|}
\hline & & & \\
\hline Spearman-Rho & Anzahl_OP & Korrelationskoeffizient & 1,000 &,$- 851^{\prime \prime}$ \\
& & Sig. (2-seitig) &. &, 000 \\
& $\mathrm{~N}$ & 18 & 18 \\
\cline { 2 - 5 } & Zeit_daVinci & Korrelationskoeffizient &,$- 851^{\mathrm{m}}$ & 1,000 \\
& Sig. (2-seitig) &, 000 &. \\
& $\mathrm{~N}$ & 18 & 18 \\
\hline
\end{tabular}

Mit einem Korrelationskoeffizient $\mathrm{R}=-0,851$ stellt die Korrelation zwischen der Anzahl der Operation und der Da-Vinci-Operationszeit in der Effektstärke nach Cohen einen sehr starken Effekt dar.

Tabelle 8: Korrelationsanalyse der Da-Vinci-Operationszeiten mit der Operationsanzahl.

norreiationen

\begin{tabular}{|lll|r|r|}
\hline & & Anzahl_OP & Zeit_Gesamt \\
\hline Spearman-Rho & Anzahl_OP & Korrelationskoeffizient & 1,000 &,- 444 \\
& & Sig. (2-seitig) &. &, 065 \\
& $\mathrm{~N}$ & 18 & 18 \\
\cline { 2 - 5 } & Zeit_Gesamt & Korrelationskoeffizient &,- 444 & 1,000 \\
& Sig. (2-seitig) &, 065 &. \\
& $\mathrm{~N}$ & 18 & 18 \\
& & &
\end{tabular}

Mit einem Korrelationskoeffizient $\mathrm{R}=-0,444$ stellt die Korrelation zwischen der Anzahl der Operation und der Gesamtoperationszeit in der Effektstärke nach Cohen einen mittleren Effekt dar.

Beide Ergebnisse sind somit groß genug um sie als bedeutend einzustufen. 
In Abbildung 21 und 22 werden die Operatonszeitwerte des Roboterteils sowie des Gesamteingriffses, in Abhänigkeit der Operationsanzahl, dargestellt. Hierbei ist unter Zuhilfenahme einer Trendlinie die negative Korrelation grafisch visualisierbar.

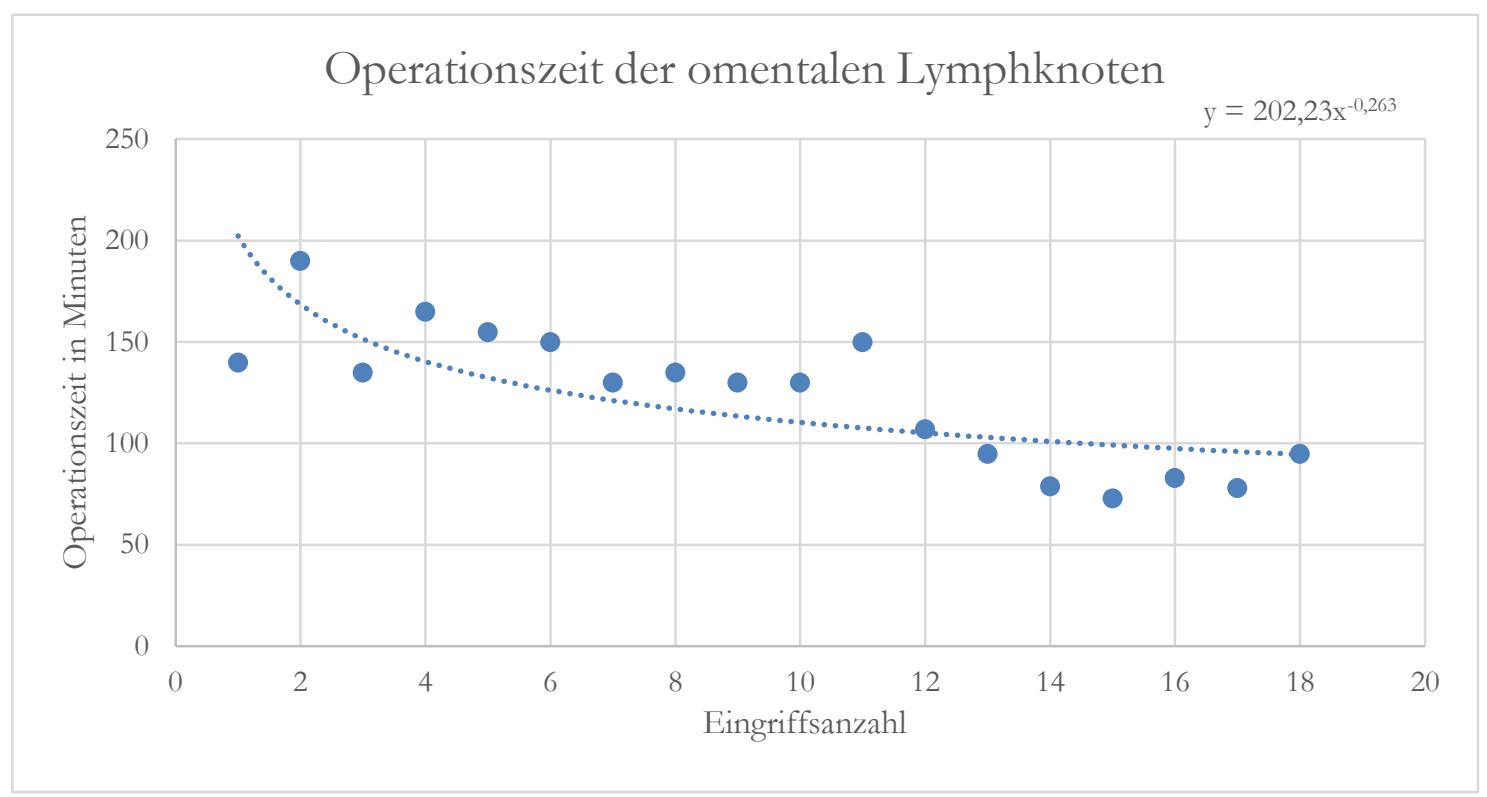

Abbildung 21: Grafische Darstellung der Operationsminuten des Da-Vinci-Systems mit potenzierte Trendlinie zur Näherung des Kurvenverlaufes.

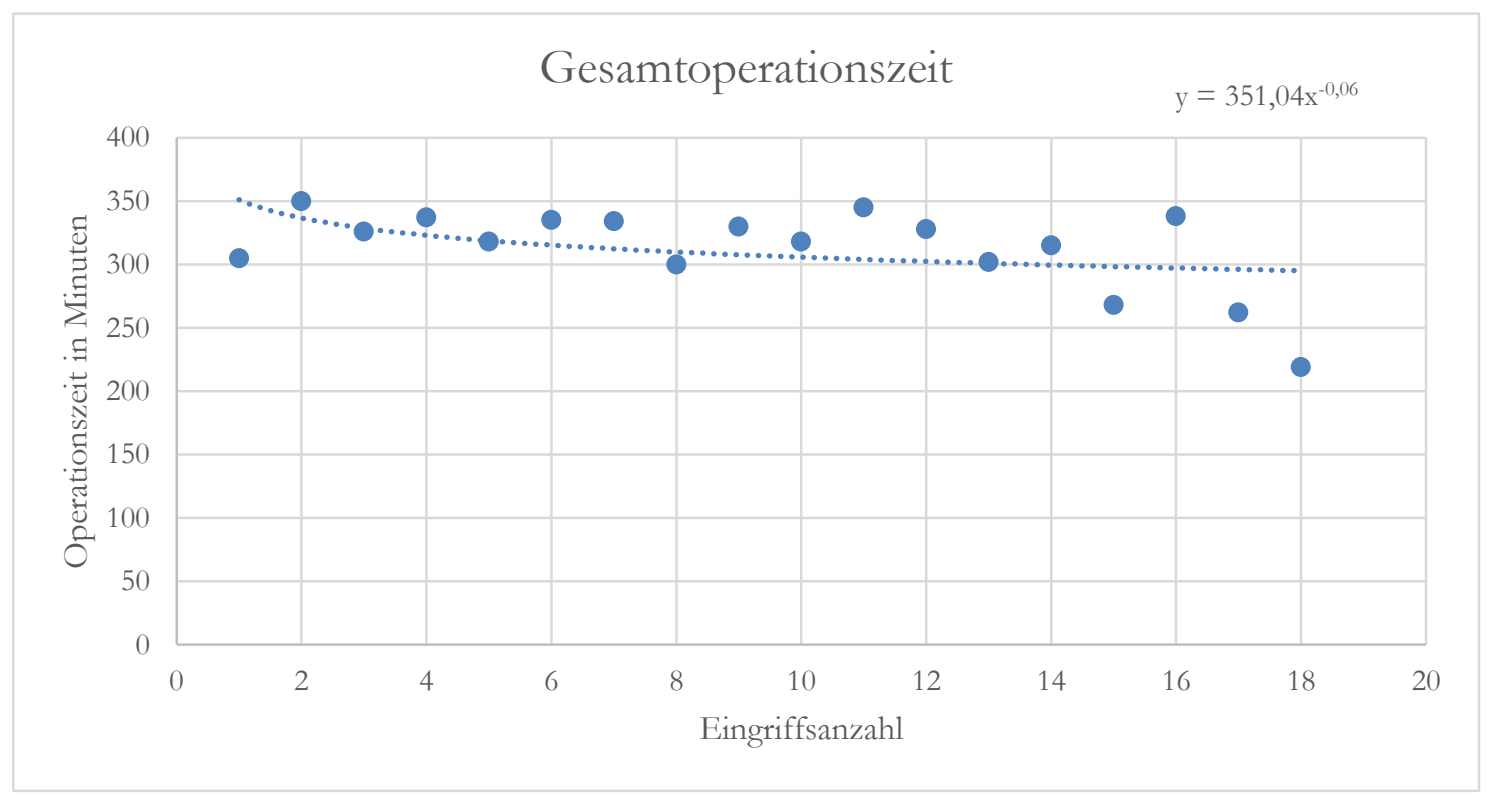

Abbildung 22: Grafische Darstellung der Gesamtoperationszeit mit potenzierte Trendlinie zur Näherung des Kurvenverlaufes. 
3.3.2.2 Autologe zervikale Lymphknotentransplantation

Es erfolgte die Korrelationsanalyse für die entsprechenden Operationszeiten mit der Rangkorrelation nach Spearman für abhängige, nicht normalverteilte Variablen. Zuvor war auch hier eine Testung auf Normalverteilung durchgeführt worden.

Tabelle 9: Korrelationsanalyse nach Spearman für die zervikalen Operationszeiten.

\section{Korrelationen}

\begin{tabular}{|c|c|c|c|c|}
\hline & & & Anzahl.oP & $\begin{array}{c}\text { Operationszei } \\
t\end{array}$ \\
\hline \multirow[t]{6}{*}{ Spearman-Rho } & \multirow[t]{3}{*}{ Anzahl.OP } & Korrelationskoeffizient & 1,000 & ,083 \\
\hline & & Sig. (2-seitig) & . &, 646 \\
\hline & & $\mathrm{N}$ & 33 & 33 \\
\hline & \multirow[t]{3}{*}{ Operationszeit } & Korrelationskoeffizient &, 083 & 1,000 \\
\hline & & Sig. (2-seitig) & ,646 & \\
\hline & & $\mathrm{N}$ & 33 & 33 \\
\hline
\end{tabular}

Mit einem Korrelationskoeffizient $\mathrm{R}=0,083$ stellt die Korrelation zwischen der Anzahl der Operation und der Operationszeit in der Effektstärke nach Cohen keinen Effekt dar.

In Abbildung 23 werden die Operatonszeitwerte in Abhänigkeit der Operationsanzahl dargestellt. Hierbei ist unter Zuhilfenahme einer Trendlinie die fehlende Korrelation grafisch visualisierbar. 


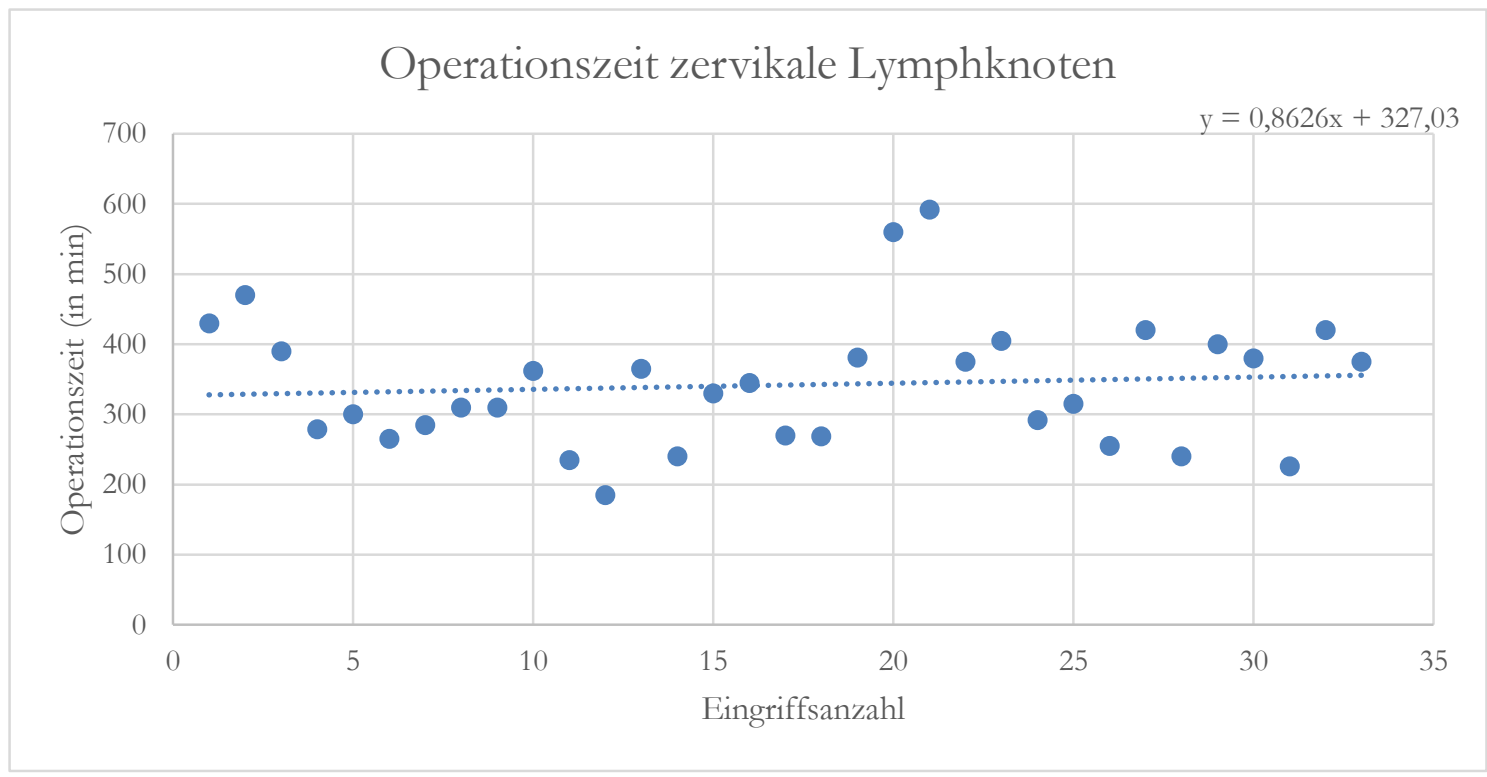

Abbildung 23: Operationszeit in Minuten der durchgeführten zervikalen Lymphknoteneingriffe mit angepasster Trendlinie und entsprechender Funktion.

\subsubsection{Lymphgefäßtransplantation}

Nach Verteilungstestung und fehlender Normalverteilung erfolgte auch für das Lymphgefäßkollektiv die Korrelationsanalyse nach Spearman für abhängige nicht normalverteilte Variablen.

Tabelle 10: Korrelationsanalyse nach Spearman für die Lymphgefäßtransplantationen.

\section{Korrelationen}

\begin{tabular}{|lll|r|r|}
\hline & & OP.Anzahl & Eingriffszeit \\
\hline Spearman-Rho & OP.Anzahl & Korrelationskoeffizient & 1,000 &, 264 \\
& & Sig. (2-seitig) &. &, 120 \\
& $\mathrm{~N}$ & 36 & 36 \\
\cline { 2 - 5 } & Eingriffszeit & Korrelationskoeffizient &, 264 & 1,000 \\
& Sig. (2-seitig) &, 120 &. \\
& $\mathrm{~N}$ & 36 & 36 \\
\hline
\end{tabular}

Mit einem Korrelationskoeffizient $\mathrm{R}=$ 0,264 stellt die Korrelation zwischen der Anzahl der Operation und der Operationszeit in der Effektstärke nach Cohen einen geringen positiven Effekt dar.

In Abbildung 24 werden die Operatonszeitwerte der Lymphgefäßtransplantation in Abhänigkeit der Operationsanzahl dargestellt. Hierbei ist unter Zuhilfenahme einer Trendlinie die Korrelation grafisch darstellbar. 


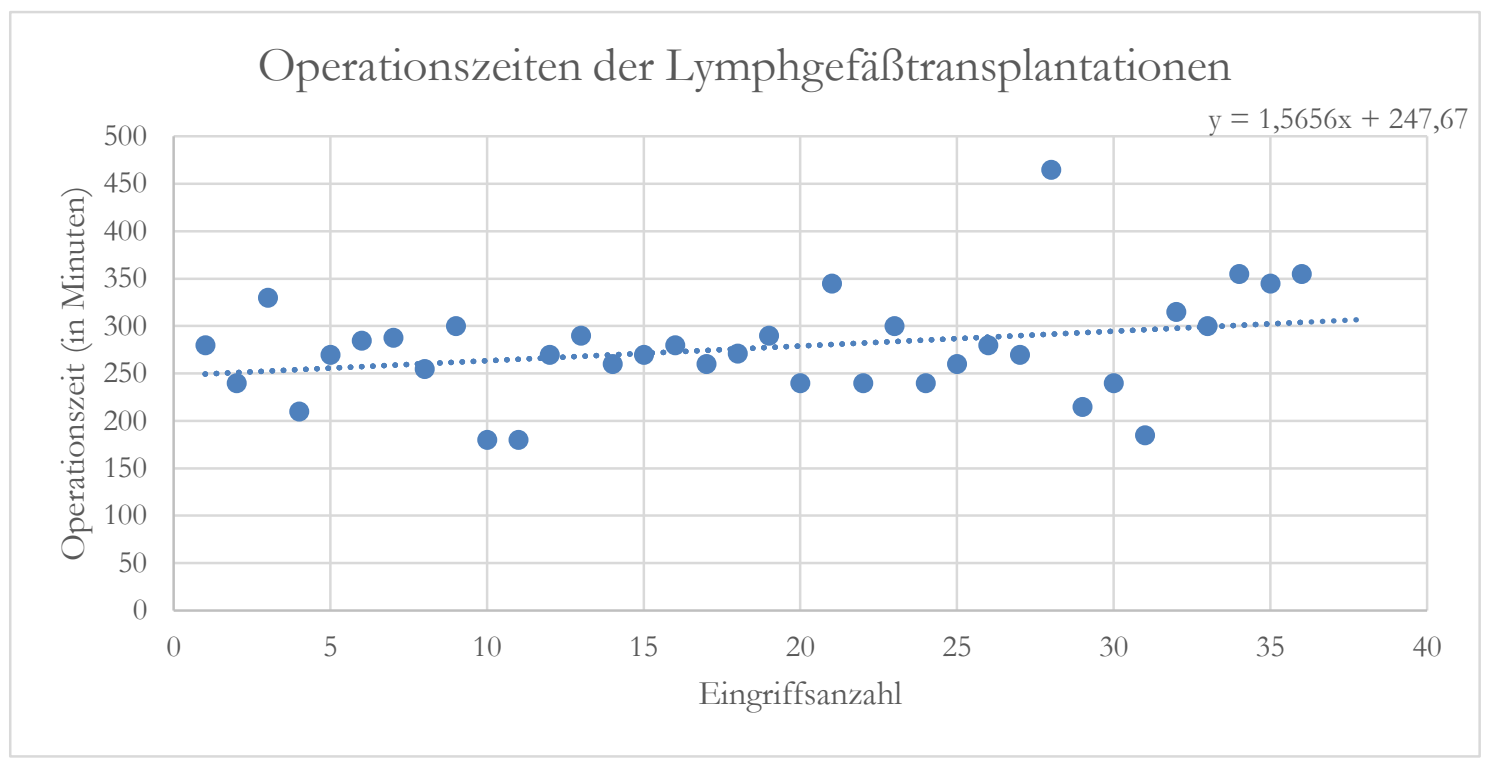

Abbildung 24: Operationszeit in Minuten der durchgeführten Lymphgefäßtransplantationen mit angepasster Trendlinie und entsprechender Funktion. 


\section{Diskussion}

\subsection{Umfangsmessung}

Durch die technische Weiterentwicklung in den letzten Jahren hat auch die Diagnostik in der Lymphologie einen deutlichen Zugewinn an Verfahren verzeichnen können. Wie bereits in der Einleitung aufgelistet, gehören hierzu unter anderem die Umfangs- und Volumenmessung, die indirekte Lymphographie, die Lymphszintigraphie sowie auch die Magnetresonanzlymphographie. Alle Verfahren, ausgenommen die reine Umfangs- und Volumenmessung, sind durch die Notwendigkeit einer Kontrastmittel- oder Tracer Applikation recht invasive und teilweise langwierige diagnostische Verfahren.

Den aktuellen Goldstandard in der Diagnostik stellt immer noch die Lymphszintigraphie dar (Cambria et al. 1993). Unsere Intention in dieser Untersuchung war es aber ein diagnostisches Verfahren zu verwenden, was sich mit der Vorstellung der Patienten in unseren Sprechstunden leicht vereinbaren lässt und gleichwohl reproduzierbar bleibt.

Auch aufgrund der Tatsache, dass die Lymphszintigraphie eine nennenswerte Strahlenbelastung mit sich bringt, stark untersucherabhängig ist und einen entsprechenden Zeitaufwand bedarf, ist dieses Verfahren für die regelmäßigen, teilweise mehrmals pro Quartal durchgeführten Nachkontrollen in unseren Sprechstunden nicht geeignet (Tiedjen et al. 2002; Herpertz 2010; Brauer 2005). Daher führten wir, in regelmäßigen Abständen bei den Patienten mit omentaler Lymphknotentransplantation, eine Umfangsmessung der entsprechenden Extremität durch. Die Umfangsmessung ist ein schnelles, einfaches und kostengünstiges Verfahren, was schon seit langer Zeit in der Lymphologie eingesetzt wird. Gleichwohl sind wir uns sehr bewusst, dass die reine Umfangsmessung nicht ohne weitere additive Messverfahren verwendet werden sollte (Smoot et al. 2011; Sawan et al. 2009). Wie bereits in der Einleitung ausführlich dargestellt, kommt es bei einem länger bestehenden Lymphödem zu chronischen Umbauprozessen im Bindegewebe mit verstärkter Fibrosierung und Fetteinlagerung (Swartz 2001). Somit kann nach mikrochirurgischen Eingriffen am Lymphgefäßsystem ein verbesserter Abfluss der Lymphe vorliegen und das Gewebe deutlich gelockert sein. In den Messwerten spiegelt sich dies jedoch nur bedingt oder gar nicht wieder. Bestärkt wird diese Vermutung durch die Tatsache, dass eine Zunahme des Wassergehaltes in der Haut um 20\% lediglich zu einer Umfangszunahme der Extremität um 2\% führt (Lahtinen et al. 2015). Einen nicht unerheblichen Anteil an der 
Umfangs Zu- oder Abnahme des Beines stellen auch Gewichtsschwankungen der Patienten dar (Mikes et al. 1999). Daher wird die Berücksichtigung des BMI oder die Verwendung eines speziellen Lymphödem Index empfohlen (Yamamoto et al. 2011a).

Die Abmessung der Umfangswerte mit dem Maßband stellt immer auch eine subjektive Messmethode dar (Sawan et al. 2009). Aus eigener Erfahrung wissen wir, dass durch minimal gesteigerten Zug am Maßband, Ungenauigkeiten der Umfangsmessung theoretisch möglich sind.

Nichtsdestotrotz sollten die Umfangswerte, bei der aktuell noch recht neuen und wenig durchgeführten Operationsmethode mit dem Da-Vinci-System, der Vollständigkeit halber in dieser Arbeit mit aufgeführt werden. Die Kombinierbarkeit der Umfangsmesswerte mit weiteren Verfahren, wie beispielsweise der Bioimpedanz oder dem prozentualen Wassergehalt der Haut, haben sich in bereits durchgeführten Studien an unserer Klinik als verlässlich, jedoch bei großer Patientenanzahl als schwierig in die Sprechstundentätigkeit integrierbar erwiesen (Stepniewski et al. 2019). Schlussendlich entsprechen die von uns erhobenen Messwerte hier den bereits gemachten Erfahrungen bei unserem zervikalen Lymphknotenkollektiv (Stepniewski et al. 2019).

Beim Vergleich der Umfangswerte sollte nicht unerwähnt bleiben, dass eine recht große Anzahl unseres omentalen Lymphknotenkollektivs weder Kompression noch Manuelle Lymphdrainage postoperativ durchführt. Logischerweise kommt es, bei fehlender MLD und Kompressionsbestrumpfung, zwangsläufig, alleine durch die Pathophysiologie des Lymphödems begründet, zu größeren Umfangswerten. Wenn die operierten Patienten langjährige Kompression getragen haben und MLD durchgeführt wurde, so sind stabile Umfangswerte und klinisch weiche Extremitäten, bei fehlender Kompression und MLD ein Jahr postoperativ, bereits ein großer Erfolg. Die postoperativ erhobenen Messwerte wären nur dann sicher mit den präoperativ erhobenen Messwerten vergleichbar, wenn Kompression und MLD konsequent nach dem chirurgischen Eingriff weitergeführt werden. 


\subsection{Mikrochirurgische Verfahren und ihre Hebedefekte}

\subsubsection{Autologe omentale Lymphknotentransplantation roboterassistiert}

Bereits in den 1974er Jahren beschäftige Goldsmith sich mit der Idee der Nutzung von omentalen Lymphknoten, im Sinne einer Transposition bei Patienten mit chronischen Lymphödemen. Für ihn war der Zugangsweg jedoch nur mit einem großen Bauchschnitt in Kombination mit einer offenen Laparotomie möglich. Dem Autor nach waren die postoperativen Ergebnisse gut, jedoch kam es teilweise zu weitreichenden Komplikationen, an deren Ende häufig der Tod des Patienten stand (Goldsmith 1974). Seit Etablierung der laparoskopischen minimalinvasiven Chirurgie in den klinischen Alltag und zuletzt der Weiterentwicklung mittels roboterassisterter Verfahren, haben sich deutliche Veränderungen in der rekonstruktiven Lymphchirurgie gezeigt. Der roboterassistierte Lymphknotentransfer aus dem Omentum majus wurde 2016 erstmals von Ciudad beschrieben (Ciudad et al. 2016). Bis zu diesem Zeitpunkt hatte selbiger Autor die omentalen Lymphknotentransplantationen in rein laparoskopischer Art und Weise durchgeführt und hiermit bei zehn Patienten bereits gute Erfolge erzielt (Ciudad et al. 2017b). Besondere Vorteile des Roboterverfahrens zeigten sich unter anderem aufgrund der dreidimensionalen Bildqualität und der robotergestützten Präparation, die den physiologischen Tremor eliminiert, und somit ein sehr präzises und gefäßschonendes Arbeiten ermöglicht (Ciudad et al. 2016).

Aufgrund der Tatsache, dass abdominelle Lymphknoten zur Transplantation verwendet werden und das Peritoneum eröffnet wird, ergeben sich mit den Eingriffen eine Vielzahl von potentiellen Komplikationen. Über diese Risiken sollten sich der behandelnde Arzt sowie auch der Patient im Klaren sein. Kategorisch lassen sich Zugangskomplikationen, Komplikationen des Pneumoperitoneums, Komplikationen des operativen Eingriffs und postoperative Komplikationen abgrenzen. Alles in allem muss gesagt werden, dass die Komplikationsraten bei laparoskopischen Eingriffen sehr gering sind. Die Hälfte aller Komplikationen entsteht bereits beim Einbringen der Trokare, zu Beginn des Eingriffs (Magrina 2002).

Bei unseren Operationen wird eine direkte Eröffnung des Peritoneums unter Sicht mit Trokareinlage durch die Kollegen der Allgemein- und Viszeralchirurgie durchgeführt. Jansen et al. untersuchten in einer gynäkologischen Multicenterstudie die möglichen Komplikationen beim laparoskopischen Zugang. Bei 25764 Patienten zeigten sich 145 Komplikationen, was einer Komplikationsrate von 0,3\% entspricht (Jansen et al. 1997). 
Andere Autoren geben Werte unterhalb von 1\% an (Ahmad et al. 2012; Jiang et al. 2012; Kirchhoff et al. 2008; Molloy et al. 2002). Als häufigste Komplikation wurden Blutungen der epigastrischen Venen und Verletzungen des Darms beschrieben (Jansen et al. 1997). In weiteren Untersuchungen konnte gezeigt werden, dass Komplikationen beim Einbringen der Trokare im Allgemeinen sehr selten sind. Durch das offene Einbringen des ersten Trokars kann die Wahrscheinlichkeit des Auftretens von Komplikationen noch verringert werden. Zusätzlich zeigen sich beim offene Einbringen weniger Wundinfekte (Perugini und Callery 2001; Nuzzo et al. 1997; Mayol et al. 1997).

Aber nicht nur die chirurgischen Komplikationen müssen in der Laparoskopie berücksichtigt werden. Eine Vielzahl von Untersuchungen haben gezeigt, dass aus der Anlage des Pneumoperitoneums, besonders bei kardial vorerkrankten Patienten, eine Beeinflussung des Herzzeitvolumens und Erhöhung des systemischen Gefäßwiderstandes resultieren kann. Dies kann konsekutiv zu schwerwiegenden Komplikationen führen (Maerz et al. 2017; Hein et al. 1997; Harris et al. 1996). Eine Vorselektion, gute Indikationsstellung und mögliche Intensivkapazität sind hier unabdingbar.

Nach dem erfolgreichen Einbringen der Trokare ist bei der weiteren Präparation dann vor allem die Erfahrung des Operateurs ein wesentlicher Faktor für das Auftreten von Komplikationen (Bennett et al. 1997). Mögliche Risiken sind hierbei die akzidentielle Gefäßverletzung oder die Perforation der Hohlorgane des Gastrointestinaltraktes.

Nach erfolgreichem operativen Eingriff sind, neben den frühpostoperativen Komplikationen wie Wundinfektionen und Wundheilungsstörungen, vor allem Trokarhernien und Verwachsungen erwähnenswert. Bensley et al. untersuchten das Auftreten von Trokarhernien und Verwachsungen nach offenen sowie laparoskopischen Eingriffen jeweils zwei und fünf Jahre postoperativ. Für Trokarhernien wurde eine Inzidenz von 1,9\%, beziehungsweise 3,2\%, angegeben. Postoperative Verwachsungen beliefen sich nach Angaben der Autoren auf 4,6\%, beziehungsweise 10,7\% (Bensley et al. 2013; Stüben et al. 2015).

Über die allgemeinen Komplikationen der Laparoskopie hinaus gibt es immer wieder auch beschriebene Komplikationen, die direkt mit dem Robotersystem zusammenhängen. Alemzadeh et al. haben daher an die Food and Drug Administration (FDA) berichtete Fehlermeldungen der Jahre 2000-2013 ausgewertet, die während beziehungsweise nach roboterunterstützten Eingriffen erfasst wurden. Bei 1.745.000 robotergestützten Eingriffen in den USA wurden 10.624 Fälle gemeldet, was eine Komplikationsrate von weniger als 0,6\% ergibt. Von diesen 10.624 Fällen wurden 1.535 (15,4\%) als unerwünschte Ereignisse 
mit signifikanter, negativer Auswirkung auf den Patienten klassifiziert. Hierunter fielen 1.391 Fälle von Patientenverletzung sowie 144 Todesfälle (Alemzadeh et al. 2016). In der Auswertung zeigte sich, dass die Komplikationsraten über die Jahre recht konstant geblieben waren, obwohl sich die Anzahl der Eingriffe deutlich gesteigert hatte. Weiter waren komplexere Eingriffe, besonders in Herz-Thorax Chirurgie sowie Kopf-Hals Chirurgie, eher zu Komplikationen prädestiniert. Als Erklärung wird die verminderte Routine bei geringeren Operationszahlen mit dem System in diesen Fachbereichen angeführt (Alemzadeh et al. 2016).

Insgesamt wurden in unserer Klinik 18 Patienten einer Lymphknotentransplantation zugeführt. Der Erhebungszeitraum erstreckte sich von November 2017 bis Januar 2020. Eine geringe Anzahl dieser Patienten berichtete über ziehende Schmerzen in der Bauchund Thoraxregion. Am ehesten ist dies auf das temporär angelegte Pneumoperitoneum zurückzuführen. Die Beschwerden waren größtenteils bereits im stationären Verlauf komplett rückläufig. Andere Komplikationen, als die bereits weiter oben erwähnten, traten in unserem Patientenkollektiv bis dato nicht auf. Zumindest was Langzeitkomplikationen wie Trokarhernien und Verwachsungen angeht, kann aufgrund der kurzen Zeit noch keine sichere Beurteilung stattfinden.

Frey et al. publizierten 2020 eine Nachuntersuchung mit einem Patientenkollektiv von $\mathrm{N}=$ 5 durchgeführten omentalen Lymphknotentransplantationen. Das Kollektiv bestand aus drei sekundären Lymphödemen nach Mammakarzinom, jeweils einem Patienten mit primärem Lymphödem der unteren Extremität sowie einem Patienten nach partieller Penektomie, Lymphonodektomie und konsekutivem Lymphödem beider Beine und dem Skrotum. Die Lymphknotentransplantation wurde in vier Fällen mit dem Da-Vinci-XiSystem durchgeführt. In einem Fall wurde ein Single Port/ SP System von Intuitive Surgical verwendet. Die gehobenen Lymphknoten wurden sowohl in anatomische $(\mathrm{N}=3)$ als auch in nichtanatomische Regionen $(\mathrm{N}=2)$ transplantiert. Hebedefekte im Bereich der abdominellen Region wurden nicht beschrieben. Zwei der fünf operierten Patienten zeigten postoperative Cellulitiden im Sinne einer Entzündung. Unter antibiotischer Therapie war dies jedoch gut rückläufig und ohne weiteren Interventionsbedarf (Frey et al. 2020). Ciudad et al. beschreiben einen Fall einer erfolgreich durchgeführten roboterassistierten omentalen Lymphknotentransplantation ohne Komplikationen (Ciudad et al. 2016).

Nach ausführlicher Literaturrecherche der aktuell in der MEDLINE Database unter diesem Themenbereich gelisteten Artikel, stellt unser Patientenkollektiv mit 18 komplikationslos operierten Patienten und elf nachuntersuchten Patienten, mit einem 
Nachuntersuchungszeitraum von mehr als 12 Monaten, das größte roboterassistierte Kollektiv dar. Die zuvor erläuterten, möglichen Komplikationen bei abdominellen laparoskopischen Eingriffen traten nicht auf. Langzeitkomplikationen wie Trokarhernien oder ein sich durch Verwachsungen entwickelnder Ileus sind laut Literatur beschriebene und mögliche Folgen der Eingriffe. Da seit der erstmaligen Durchführung des Eingriffes an unserer Klinik (November 2017) erst drei Jahre vergangen sind, können Langzeitfolgen noch nicht abschließend beurteilt werden.

Wie bereits von Frey et al. beschrieben und durchgeführt, werden in der Literatur auch immer wieder Transplantationen von Lymphknoten in nichtanatomische Regionen, wie den Ellenbogen, das Sprunggelenk oder den Fußrücken gefunden. Die Idee der Autoren besteht darin die bereits in der Peripherie gestaute Lymphflüssigkeit in das venöse System abzuleiten, ohne dass ein Transport gegen die Schwerkraft notwendig ist (Frey et al. 2020; Cheng et al. 2012; Sapountzis et al. 2014a). Ein Großteil der Patienten mit Lymphödem wird postoperativ einer Radiatio zugeführt. Ein weiterer Vorteil der Transplantation in nichtanatomische, periphere Regionen liegt somit in der gesunden Beschaffenheit des Gewebes ohne Strahlenschäden, Verwachsungen oder Vernarbungen. Die Indikation für die Transplantation in periphere Regionen sollte jedoch stets gut geprüft und mit den Patienten besprochen werden. Ein primärer Hautverschluss über dem Transplantat gelingt aufgrund des dünnen Haut-Weichteilmantels in diesen Regionen fast nie. Zwar kann im Verlauf eine Lappenausdünnung vorgenommen werden (Cheng et al. 2012), eine visuelle sowie teilweise funktionelle Einschränkung wird nach solchen Transplantationen jedoch häufig verbleiben.

Im Gegensatz dazu ist bei der inguinalen oder axillären Lymphknotentransplantation ein primärerer Hautverschluss fast immer ohne funktionelle Einschränkungen möglich. Das durch die Bestrahlung und Voroperation häufig vernarbte Gewebe sollte entfernt und gelöst werden, um ein lockeres und entspanntes Transplantatbett zu gewährleisten (Becker et al. 2012).

Durch verschiedenen klinische sowie experimentelle Untersuchungen konnte dargestellt werden, dass vaskularisierte Lymphknoten nach erfolgreicher mikrochirurgischer Gefäßnaht und Reperfusion des Transplantatlappens durch Gefäßneubildung, Anschluss an die entsprechende Empfängerregion erlangen können. Tobbia et al. untersuchten an einem Schafsmodell, ob die Transplantation eines autologen Lymphknotens in eine lymphonodektomierte Region die lymphatische Transportfunktion wiederherstellen und das Ausmaß des postoperativen Lymphödems reduzieren würde. Es wurden jeweils 
vaskularisierte und avaskularisierte Lymphknoten transplantiert. In einer dritten Gruppe wurde ein reiner Hautschnitt ohne Lymphknotentransplantation durchgeführt. Die vaskularisierten Lymphknotentransplantate waren mit der größten klinischen Verbesserung assoziiert, wobei sich eine signifikante Ödemreduktion und eine deutlich bessere Lymphtransportfähigkeit, im Vergleich zu den beiden anderen Gruppen, zeigte (Tobbia et al. 2009). Somit ist die Vaskularisierung des Transplantates für den postoperativen Erfolg des Eingriffes ein essentieller Faktor. Eine direkte Anastomisierung der Lymphknoten mit entsprechenden Lymphgefäßen ist für die Funktionsaufnahme jedoch nicht notwendig (Miranda Garcés et al. 2017; Cheng et al. 2014). Nach Maruyama et al. besitzen Makrophagen die Möglichkeit mithilfe von VEGF-C (vascular endothelial growth factor $C$ ) eine Metamorphose zu lymphatischen Endothelzellen zu durchlaufen und ermöglichen dadurch nach einer gewissen Zeit den Anschluss der transplantierten Lymphknoten an das umliegende Gewebe (Aschen et al. 2014; Yan et al. 2011). VEGF-C stellt den Liganden für den VEGFR 3 (vascular endothelial growth factor receptor 3) dar und ist mit ihm an der zellulären Neubildung der Lymphgefäße beteiligt (Aschen et al. 2014; Yan et al. 2011).

Mit der Fragestellung nach lymphangiogener Revaskularisierung und deren Mechanismen transplantierten Yan et al. in einem Tiermodell Hautlappen auf entsprechende Mäuseschwänze, bei denen zuvor ein $2 \mathrm{~mm}$ langer Hautschnitt mit Präparation und Ligatur der Lymphgefäße durchgeführt worden war. Verglichen wurden die Ergebnisse mit einer Kontrollgruppe, bei der ein reiner Hautschnitt mit Ligatur der Lymphgefäße im Bereich der Schwanzregion stattgefunden hatte (Yan et al. 2011). In der Untersuchung des VEGFC Verteilungsmusters zwei Wochen nach Gewebetransfer zeigten sich in der immunhistologischen Färbung die höchsten Expressionen in der Peripherie des Lappens. Nach weiteren sechs Wochen war die höchste Expression dann in der zentralen Region des Transplantates zu erkennen. Nach Yan et al. zeigte das von peripher nach zentral verlaufende VEGF-C Expressionsmuster die Infiltration der Lymphgefäße, beginnend vom peripheren Rand des Hautlappens in das Zentrum. An der Lymphangiogenese waren hauptsächlich die von der Empfängermaus stammenden Lymphgefäße beteiligt. Darüber hinaus schienen die Lymphgefäße des Spenders spontan mit denen des Empfängers zu reanastomosieren. Im Vergleich zur Kontrollgruppe zeigte sich eine vierfache Abnahme des Schwanzvolumens sowie eine in der Lymphszintigraphie diagnostizierte 2,5- fache Zunahme der lymphatischen Transportgeschwindigkeit (Yan et al. 2011).

An einem Mausmodell versuchen Aschen et al. die Herkunft der, in die transplantierten Lymphknoten infiltrierenden, Lymphgefäße zu evaluieren. In immunohistologischen 
Auswertungen wurde zusätzlich die VEGF-C Expression sowie die Lokalisation der eingewachsenen Lymphgefäße untersucht. In den infiltrierenden Lymphgefäßen und im perinodalen Fett war es zu einer deutlichen Zunahme der VEGF-C Expression gekommen. Neu gebildete Lymphgefäße lagen in unmittelbarer Nähe zu den hochendothelialen Venolen der Lymphknoten und die transplantierten Mäuse zeigten bereits vier Wochen nach Transplantation eine fast gleiche Isotopenleuchtkraft des Technetium-99, im Vergleich mit der Kontrollgruppe. Laut den Autoren konnte somit eine schnelle, von den Endothelzellen des umliegenden Gewebes ausgehende, Infiltration von funktionellen Lymphgefäßen in die Lymphknoten bewiesen werden. In Korrelation mit der Infiltration aus der Peripherie konnte eine erhöhte endogene Expression von VEGF-C im perinodalen Fett und in den infiltrierenden Lymphgefäßen gezeigt werden. Durch die anatomische Nähe der neu gebildeten Lymphgefäße zu den hochendothelialen Venolen der Lymphknoten sahen Aschen et al. die Hypothese unterstützt, dass der Lymphknotentransfer das entsprechende Lymphödem durch Austausch mit der systemischen Zirkulation, im Sinne von lympho-venösen Anastomosen, verbessert (Aschen et al. 2014).

Trotz den oben beschriebenen postoperativen Erfolgen nach autologer Lymphknotentransplantation kommt es immer wieder zu nicht komplett suffizienter Entstauung im Bereich der operierten Extremität. Becker et al. beobachteten dies mehrfach im Bereich des Ellenbogens und führten dann eine erneute Lymphknotentransplantation von der kontralateralen Seite der initial verwendeten Leistenseite durch (Becker et al. 2012). Auch wir beobachteten in unserem omentalen roboterassistierten Patientenkollektiv in einigen Fällen eine residuelle Schwellung mit Verhärtung im Bereich des Ellenbogens und der dorsalen proximalen Unterarmregion. Wir führten bei Beschwerdepersistenz und Operationswunsch die Anlage von Lympho-venösen Anastomosen durch. Aufgrund des geringen Risikoprofil der LVAs sind wir sicher, dass ein zusätzlicher Schaden bei guten postoperativen Ergebnissen ausbleibt und die entsprechenden Regionen gezielt entstaut werden können (Klingelhoefer et al. 2019). Eine spezifische Nachuntersuchung der LVAs, als ergänzender Eingriff zur roboterassistierten Lymphknotentransplantation, ist zum aktuellen Zeitpunkt an unserer Klinik noch ausstehend.

\subsubsection{Autologe zervikale Lymphknotentransplantation}

Die autologe Lymphknotentransplantation ist ein mikrochirurgisches Verfahren, was durch C. Becker in den 1980er Jahren etabliert und geprägt wurde. Die Idee dahinter besteht in der Umsetzung von nicht bestrahlten Lymphknoten, mit ihrem umgebenden Fettgewebe 
und versorgenden Gefäßen, in die entsprechende lymphödematös veränderte Empfängerregion. Hier soll durch eine zusätzliche Narbenlösung und Implantation der gesunden Lymphknoten die Blockierung des Lymphabflusses, welcher durch Primäroperation, Bestrahlung und Lymphknotenentfernung verursacht wurde, aufgehoben werden (Becker et al. 2012; Becker et al. 2006; Becker et al. 1991). An sich funktionieren die Lymphknoten dann im Sinne einer Kurzschlussverbindung zwischen Lymphgefäß beziehungsweise Lymphknoten und Venen. Nach Lin et al. sowie Cheng et al. wird die Lymphflüssigkeit dann über die hochendothelialen Venolen dem venösen System zugeführt und abtransportiert (Cheng et al. 2014; Lin et al. 2009).

Über postoperative Ergebnisse der Lymphknotentransplantation sind bereits eine Vielzahl von Publikationen bekannt. Becker et al. beschrieben 2006 die postoperativen Resultate von 24 Patienten, die nach einer Brustkrebserkrankung und Axilladissektion einer Lymphknotentransplantation zugeführt wurden. Als Spenderregion wurden hier vaskularisierte Lymphknoten der Leiste verwendet, die in die Axilla transplantiert wurden. Nach 8,3 Jahren mittlerem Beobachtungszeitraum hatten sich die Extremitätenumfänge in zehn Fällen der kontralateralen, gesunden Seite angeglichen. Bei 12 Patienten kam es zu einer Reduktion der Extremitätenumfänge um 50\%. Lediglich bei zwei Patienten zeigte sich keine Veränderung nach operativer Versorgung (Becker et al. 2006). Eine gesonderte Darstellung oder Auflistung von Komplikationen wird nicht gegeben.

Cheng et al. führten bei sechs Patienten mit sekundären Beinlymphödemen eine Lymphknotentransplantation mit submentalen Lymphknotenpaketen als Spenderregion durch und transplantierten diese in die Sprunggelenksregion. Im Ergebnis konnten signifikante Volumendifferenzen zwischen den präoperativen und postoperativen Volumenmessungen gezeigt werden. Zusätzlich kam es zu einer Reduktion der Erysipelneigung (Cheng et al. 2012). Prinzipiell gibt es eine Vielzahl von möglichen Spenderregionen. Viele Autoren beziehungsweise Chirurgen bevorzugen aber die Entnahme von supraklavikulären Lymphknoten, anstatt von oben genannten submentalen Lymphknoten, da hierbei häufig eine deutlich dezentere Narbe resultiert (Althubaiti et al. 2013). Zusätzlich birgt die Entnahme in der Submentalregion die Gefahr einer intraoperativen Schädigung des Ramus marginalis mandibulae des Nervus facialis. Bei der supraklavikulären Entnahme wird dieser Risikofaktor umgangen (Cheng et al. 2012).

Auch Batista et al. transplantierten von 1990 bis 201238 Patienten mit sekundären Beinlymphödemen. 30 von 38 Patienten hatten eine inguinale Lymphonodektomie und 27 von 38 hatten eine Radiatio erhalten. In der Mehrzahl der Fälle wurde als Spenderstelle die 
laterale Thoraxwand verwendet, in etwas weniger Fällen die kontralaterale Zervikal- oder Inguinalregion. Die postoperativen Ergebnisse zeigten bei 36 von 38 Patienten eine Volumenreduktion von mindestens 30\% (Batista et al. 2017).

Ciudad et al. haben 2017 verschiedene Hebedefekte bei 110 Patienten, welche über zwei Jahre postoperativ betreut wurden, nachuntersucht. Elf Patienten hatten ein primäres Lymphödem, während 99 an einem sekundären Lymphödem litten. In die Auswertung konnten 69 Patienten mit Lymphödem der unteren Extremität und 41 mit Lymphödem der oberen Extremität eingeschlossen werden. Als Spenderregion wurden inguinale, supraklavikuläre und omentale Entnahmen durchgeführt. Ciudad beschreibt im ganzen Beobachtungszeitraum nur drei Hebedefektkomplikationen. Zweimal zeigten sich Lymphfisteln mit Austritt von Lymphflüssigkeit, jeweils nach inguinaler und supraklavikulärer Lymphknotenentnahme. Bei einem Patienten kam es direkt nach supraklavikulärer Lymphknotenentnahme zur Nachblutung, die chirurgisch, durch eine Ligatur der Arteria transversa colli, gestillt wurde (Ciudad et al. 2017a).

Allgemein sollte es sich bei den Spenderlymphknoten möglichst um gesunde, unbestrahlte Lymphknoten handeln, damit die optimalen Bedingungen für die zügige und vollumfängliche Funktionsaufnahme gewährleistet ist. Besonders bei sekundären Lymphödemen der unteren Extremität, bei denen eine Radiatio der entsprechenden Region und des kleinen Beckens sehr wahrscheinlich ist, sollte die Entnahme von kontralateralen inguinalen Lymphknoten sorgfältig geprüft werden. Das Risiko eines iatrogenen Lymphödems der Spenderregion zeigt sich in diesen Fällen aufgrund der potentiellen Vorschädigung deutlich erhöht (Pons et al. 2014; Vignes et al. 2013).

Nach ausführlicher Literaturrecherche existiert zum aktuellen Zeitpunkt nur eine Veröffentlichung über die Entwicklung von iatrogenen Armlymphödemen, im Sinne eines Hebedefekts, nach der Entnahme eines supraklavikulären Lymphknotenpaketes (Lee et al. 2015). Maldonado et al. untersuchten 2017 die Hebedefektmorbidität von supraklavikulären Lymphknoten. Die Untersuchungsgruppe bestand aus 100 Patienten. 22 von 100 Patienten litten an einem primären, die anderen 78 der 100 an einem sekundären Lymphödem. Bei 16 Patienten wurde das Lymphknotenpaket mit entsprechender Monitorinsel gehoben und als Transplantat in der Axilla oder Leistenregion verwendet. Bei drei Patienten kam es zu einer Leckage im Sinne einer Lymphfistel, welche sich ohne chirurgischen Handlungsbedarf verschloss. Bei zwei Patienten traten Wundinfektionen in der supraklavikulären Region auf. Einmalig entwickelte sich postoperativ eine Lymphozele, die dann durch einen erneuten Eingriff versorgt werden musste. Im ersten postoperativen 
Monat klagte eine geringe Anzahl von Patienten über leichte Parästhesien im Bereich der Zugangsnarbe supraklavikular (Maldonado et al. 2017).

In der Nachuntersuchung unserer Daten von Januar 2013 bis April 2019 zeigten sich ähnliche Ergebnisse. Eine Patientin musste operativ an einer Lymphfistel revidiert werden. Zwei Lymphozelen konnten erfolgreich konservativ durch Punktion behandelt werden. Selbiges Procedere galt auch für ein Serom, was jedoch mehrfach abpunktiert wurde. Mit der Anzahl von jeweils $\mathrm{N}=1$ traten in unserem Fall ein Wundinfekt und eine hypertrophe, schmerzhafte Narbe auf. Ähnlich wie Maldonado et al. können auch wir einen Fall von Hypästhesie in der supraklavikulären Region verzeichnen.

Das im Patientenkollektiv einmalig aufgetretene Horner-Syndrom verdeutlicht die mehrfach thematisierte komplexe Anatomie im Bereich der supraklavikular Region (Maldonado et al. 2017). Der Symptomkomplex aus Ptosis, Endophtahalmus und Miosis stellt mit einer Inizidenz von 0,2\% eine sehr seltene Komplikation, vor allem bei Eingriffen an der Schilddrüse und bei neck dissections, dar (Cozzaglio et al. 2008; Hu et al. 2017). Cozzaglio et al. untersuchten 495 durchgeführte Thyreoidektomien zwischen 1997 bis 2007. Mit genau einem dokumentierten Fall ergibt sich die oben genannte Inzidenz von 0,2\% (Cozzaglio et al. 2008). Bis dato ist der genaue pathophysiologische Mechanismus des Horner-Syndroms noch ungeklärt. Es schließt sich jedoch immer ein konservatives Therapieprozedere an (Sapalidis et al. 2019). Mögliche Ursachen sind postoperative Hämatome, durch Ischämie hervorgerufene Nervenschäden oder eine, durch eingesetzte Retraktoren und andere Instrumente, hervorgerufene Zugbelastung des Sympatikusnerves. Bei spontaner Regeneration, wie auch in unserem Fall, geht man von einer intraoperativen Überdehnung des entsprechenden Nervens aus (Cozzaglio et al. 2008).

Unsere eigenen Ergebnisse sowie die exemplarisch erwähnte Literatur zeigt deutlich, dass die autologe zervikale Lymphgefäßtransplantation eine wirksame und sinnvolle Möglichkeit in der Therapie des sekundären Lymphödems der Extremitäten darstellt. Komplikationen sind in der Regel selten, jedoch nicht komplett vernachlässigbar. Bezüglich der supraklavikulären Lymphknotentransplantation und unter Berücksichtigung der aktuellen Vergleichsliteratur, entsprechen unsere herausgearbeiteten Hebedefektmorbiditäten der aktuellen Datenlage.

\subsubsection{Lymphgefäßtransplantation}

Die autologe Lymphgefäßtransplantation wurde Anfang der 1980er Jahre von Baumeister erstmalig angewendet und in den folgenden Jahren fest etabliert (Baumeister et al. 1981b; 
Baumeister et al. 1981a). Sie stellt ein mikrochirurgisches Verfahren dar, bei dem Lymphkollektoren von der gesunden Extremität mit den Lymphkollektoren der aszendierenden kranken Seite verbunden werden um somit den Lymphabfluss der entsprechenden Region verbessern (Baumeister et al. 2016).

Insgesamt wurden von Baumeister et al. zwischen den Jahren 1980 bis 2011, 352 Patienten lymphgefäßtransplantiert. Von den 352 Lymphödemen entfielen 143 auf die untere Extremität. In einer Langzeitstudie aus dem Jahr 2016, zeigten sich vor allem für die obere Extremität sehr gute Ergebnisse. Bei 16 Patienten, die über einen Zeitraum von zehn Jahren nachbeobachtet wurden, resultierte eine Anpassung der Armvolumina zu den entsprechenden Messwerten einer Kontrollgruppe. Auch in der Gruppe mit Beinlymphödemen war eine signifikante Umfangsreduktion zu beobachten (Baumeister et al. 2016).

In einer vorherigen Studie in unserer Abteilung war bei 14 Patienten mit sekundärem Lymphödem eine Lymphgefäßtransplantation durchgeführt worden. Fünf von 14 waren Lymphödeme der unteren Extremität. Bei zwei dieser fünf Patienten war innerhalb des mittleren Beobachtungszeitraums von 2,6 Jahren im Seitenvergleich kein Volumenüberschuss mehr quantifizierbar (Felmerer et al. 2012).

Weiss et al. untersuchten mit Hilfe der Lymphszintigraphie die Auswirkung der Lymphgefäßtransplantation auf den Lymphabfluss der entsprechenden Empfängerextremität. Insgesamt wurden über acht Jahre hinweg 20 Patienten in regelmäßigen Abständen nachkontrolliert. Hierbei zeigte sich bei 17 von 20 Patienten ein signifikant gebesserter Lymphabfluss. Bei zwei von acht Patienten zeigten sich normwertige Lymphabflusswerte (Weiss et al. 1996; Weiss et al. 2003).

Auch die in unserer Klinik zwischen März 2010 und September 2019 durchgeführten Eingriffe zeigen sowohl klinisch als auch im Bedarf der konservativen Therapie zufriedenstellende Ergebnisse. Oben genannte Studien unterstreichen die allgemeine Praktikabilität der Lymphgefäßtransplantation. Ziel dieser Untersuchung war es jedoch auch die Hebedefektmorbidität der Lymphgefäßtransplantation zu evaluieren. In der Literatur finden sich bis dato hierzu keine verlässlichen Angaben. Ob bis zum aktuellen Zeitpunkt noch nie Hebedefekte aufgetreten sind kann somit nicht sicher beurteilt werden.

In unserer Untersuchung erhoben wir insgesamt 12 Hebedefektmorbiditäten. Mit jeweils N = 1 waren Wundinfekte stationär sowie poststationär, Wundheilungsstörungen mit und ohne Hautnekrose und eine Lymphfistel vorhanden. Die entsprechende 
Wundheilungsstörung mit Hautnekrose musste operativ versorgt werden. Alle weiteren Komplikationen konnten konservativ behandelt werden. Zweimal traten Lymphfisteln, ohne notwendigen Interventionsbedarf auf, die sich spontan und nach Anhebung des Albuminspiegels verschlossen. Mit einer Anzahl von $\mathrm{N}=5 \mathrm{kam}$ es $\mathrm{zu}$ iatrogenen Lymphödeme im Bereich des Spenderbeines. Eine genaue Darstellung des benötigten Kompressionsbedarfes findet sich unter 3.1.3. im Ergebnisteil. Mit einem prozentualen Anteil von 13,88\% (5/36) liegt die Inzidenz recht hoch. Hier sollte jedoch hervorgehoben werden, dass eine entsprechende Kompression eventuell nicht von Dauer gewesen ist. Die Nennung als Komplikation in unsere Liste erfolgte bereits bei Umfangszunahme oder Rezeptierung von Kompressionskleidung am entsprechenden Bein. Eine Langzeitevaluation erfolgte hier nicht.

Die Entscheidung für eine Lymphgefäßtransplantation wurde in unserer Abteilung alleine von klinischen Faktoren abhängig gemacht. Eine bereits aufgetretene, minimale Schwellneigung am potentiellen Spenderbein sowie ein pathologisches Verteilungsmuster nach Einspritzen des Patentblaues intraoperativ waren Kontraindikationen für den Eingriff.

Der Großteil (61,11\%) unserer operierten Patienten erhielt im Zuge der onkologischen Primäroperation eine kombinierte Radiochemotherapie $(\mathrm{N}=19)$, beziehungsweise eine reine Radiotherapie $(\mathrm{N}=3$ ). Häufig kommt es bei der durchgeführten Radiotherapie zu einer entsprechenden Schädigung der ableitenden pelvinen Lymphknoten sowie entsprechender afferenter Lymphgefäße. Tatsächlich ist es sehr gut möglich, dass eine Schädigung durch fibrosklerotische Umbauprozesse nach Radiotherapie im Bereich des Spenderbeines bereits stattgefunden hatte und die Lymphgefäße deutlich in ihrer Funktion eingeschränkt waren. Trotz eingeschränkter Funktion war es aber visuell und anamnestisch nie zu einer Schwellneigung gekommen (Mihara et al. 2012b). Gestützt wird diese Vermutung von der Tatsache, dass es sich bei 3 von 5 Patienten mit iatrogenen Lymphödemen der Spenderstelle um initiale Beinlymphödeme handelt. Alle drei Patienten hatten nach primärem onkologischen Eingriff eine Radiatio im Beckenbereich erhalten. Die zwei weiteren Armlymphödeme wurden postoperativ ebenso mit einer Radiochemotherapie behandelt. Eine Strahlenschädigung der Lymphgefäße ist aufgrund der anatomischen Distanz von Axilla und Inguinalregion im Falle der Armlymphödeme eher unwahrscheinlich. Eine mögliche Erklärung für das Auftreten der iatrogenen Beinlymphödeme könnte jedoch in der durchgeführten Chemotherapie liegen. Bereits mehrere Studien haben gezeigt, dass entsprechende Chemotherapeutika, besonders in 
Kombination mit einer Lymphonodektomie, zu sekundären Lymphödemen führen oder diese deutlich begünstigen können (Hidding et al. 2018; Swaroop et al. 2015; Lee et al. 2014). In Kombination mit dem chirurgischen Eingriff und der Entnahme der Lymphgefäße sowie der damit verbundenen zusätzlichen Schädigung in der entsprechenden Region wird die Entwicklung von Lymphödemen an der Spenderregion deutlich wahrscheinlicher.

Entsprechend dieser Hypothesen sollte, zusätzlich zur Anamnese sowie der klinischen Untersuchung, ein weiteres diagnostisches Verfahren zur Darstellung und Untersuchung der Lymphgefäße berücksichtigt werden. Wie oben bereits beschrieben, stellt aktuell noch die Lymphgefäßszintigraphie das Standardverfahren in der Lymphödemdiagnostik dar (Cambria et al. 1993). Die Untersuchung von überlegeneren Verfahren sind aktuell Thema vieler Studien. Mihara et al. haben 2012 verschiedene Möglichkeiten der Lymphödemdiagnostik verglichen. Das verwendete Patientenkollektiv bestand aus sekundären einseitigen Lymphödemen des ISL-Stadium I. In der Untersuchung zeigten sich ICG-Lymphangiographie und MR-Lymphangiographie, aufgrund einer besseren Spezifität von 1,0, den anderen Verfahren überlegen. In der Auswertung zeigte die Lymphszintigraphie signifikant häufiger falsch negative Ergebnisse. Mihara et al. empfahlen daher zur präoperativen Planung einer Transplantation der Lymphgefäße eine MRLymphangiographie sowie eine ICG-Lymphographie durchzuführen. Hiermit solle zuerst der Zustand des Lymphgefäßsystems und dann der Zustand und die Veränderungen des umliegenden Gewebes beurteilt werden (Mihara et al. 2012a).

In der tatsächlichen klinischen Anwendung beinhaltet die ICG-Lymphographie jedoch auch deutliche Limitationen. Unserer Erfahrung nach und in publizierten Artikeln haben sich vor allem die geringe Eindringtiefe und das eingeschränkte Sichtfeld als problematisch erwiesen (Ogata et al. 2007).

Eine neue Methode zur Darstellung von Blut- und Lymphgefäßen könnte diesen Konflikt eventuell beheben. Giacalone et al. untersuchten 2020 in einer Pilotstudie elf Patienten mit primären und sekundären Lymphödemen mit dem MSOT Gerät, iThera Medical GmbH, München, Deutschland. In acht von elf Fällen konnten sowohl Blut- als auch Lymphgefäße dargestellt werden. In zwei von elf Fällen gelang sogar die Darstellung der pulsatilen Bewegungen der Lymphgefäße. Insgesamt war eine Tiefendarstellung von bis zu $2 \mathrm{~cm}$ möglich. Zusätzlich und mit der parallelen Möglichkeit der Darstellung von Blutgefäßen bringt die Technik laut Giacalone neue Einblicke in der Diagnostik und schlussendlich auch der Therapie des Lymphödems (Giacalone et al. 2020). Im Gegensatz zu den vorher 
genannten Verfahren, verwendet das optoakustische MSOT Verfahren keine ionisierte Strahlung und kann im Gegensatz zur Lymphszintigraphie auch räumliche Informationen liefern. Des Weiteren können tiefer gelegene Lymphgefäße dargestellt werden ohne vom Einfluss des dermal backflows, wie bei der ICG-Lymphangiographie, gestört zu werden. Die gleichzeitige Darstellung von Blut- und Lymphgefäßen ist, im Vergleich zu den anderen Verfahren, ein Alleinstellungsmerkmal. Ob sich die MSOT Technik im klinischen Alltag bewähren kann, muss in weiteren Studien gezeigt werden.

Die Ergebnisse der Auswertung unserer Lymphgefäßgruppe sowie weitere exemplarisch aufgeführte Studien zeigen, dass eine Lymphgefäßtransplantation eine Linderung der Symptome und der Extremitätenumfänge bewirken kann. Nach unseren Daten ist ein Hebedefekt bei der operativen Versorgung ein nicht zu unterschätzender Risikofaktor. Eine große Bedeutung kommt hier der Anamnese, klinischen Untersuchung sowie auch präoperativen Diagnostik zu. Neue Verfahren zur Beurteilung des Zustandes der Lymphgefäße sind Stand aktueller Forschung. Die MSOT Technik bietet hier vielversprechende Ansätze, die in Zukunft mittels weiterer Studien evaluiert werden müssen.

\subsection{Konservative Therapie}

Durch unsere langjährige Erfahrung in der rekonstruktiven Lymphchirurgie und durch die zahlreichen Gespräche mit betroffenen Patienten, wurde über die Jahre deutlich wie stark die Krankheit den Alltag der Patienten dominiert. Vorherige Untersuchungen in Form von Fragebögen haben gezeigt, dass das tägliche Tragen und Anlegen der Kompressionsbestrumpfung sowie die vielen physiotherapeutischen Intervalle eine weitreichende Einschränkung für das alltägliche Leben der Patienten bedeutet (Stepniewski et al. 2019). Mit dieser Untersuchung wollten wir deshalb auch das Maß an konservativer Therapie postoperativ darstellen. Zusätzlich zu den in Abbildung 5 bis 11 gezeigten Kreisdiagrammen soll die Tabelle 5 die entsprechende konservative Therapie nach chirurgischer Intervention übersichtlich darstellen.

Insgesamt hängt die Entwicklung des Lymphödems nach chirurgischem Eingriff von vielen verschiedenen Faktoren ab. Der Erfolg der Eingriffe ist nicht genau vorhersehbar und häufig schwierig zu objektivieren. Mögliche bedingende Faktoren sind beispielsweise die radiogene Schädigung des Gewebes, der Grad des bereits erfolgten fibrosklerotischen Umbaus und die Pflege des Lymphödems. Eine Übersicht der konservativen Therapien im 
Vergleich (Insgesamt sowie aufgeteilt nach entsprechendem Eingriff) findet sich im Ergebnissteil 3.3.3 Konservative Therapie beziehungsweise Tabelle 5.

Vorherige Untersuchungen mit einem sehr kleinen Patientenkollektiv haben bereits gezeigt, dass sich im Bezug auf die postoperativ erhobenen Umfangswerte keine statistisch verwertbaren Unterschiede zeigen lassen (Stepniewski et al. 2019). Dies konnten wir in unserer Untersuchung bestätigen.

In unserer Nachuntersuchung lassen sich, bezüglich der postoperativ notwendigen konservativen Therapie, deutliche Unterschiede zwischen den drei Verfahren ausmachen. Mit einer Anzahl von N = 36 stellt die Gruppe der Lymphgefäßtransplantationen das größte Kollektiv dar. Es resultierte aber nur bei 22,21\% eine Verbesserung der konservativen Therapie. Die zervikalen Lymphknoten liegen mit 48,48\% deutlich vor der Lymphgefäßtransplantation. Mit einer Verbesserung von $81,81 \%$ zeigt die omentale Lymphknotengruppe die besten Ergebnisse. Besonders deutlich wird dies bei Betrachtung der Anzahl der Patienten, welche nach dem Eingriff komplett ohne konservative Therapie verblieben. Obwohl in der omentalen Gruppe dreifach weniger Patienten operiert wurden, ist die Anzahl $(\mathrm{N}=4)$ der Patienten ohne konservative Therapie, verglichen mit den zervikalen Patienten, die Gleiche. Die erhobenen Werte nach Lymphgefäßtransplantation sind, aufgrund der unterschiedlichen Wirkungsweise des Eingriffes, nicht wirklich mit den beiden anderen Methoden vergleichbar. Unter Berücksichtigung der Ergebnisse ist jedoch klar, dass die Lymphgefäßtransplantationen, im Bezug auf den postoperativen Bedarf an konservativer Therapie, das schlechteste Ergebnis der drei Verfahren liefert.

Deutliche Unterschiede der beiden Lymphknotenkollektive zeigen sich bei der Anzahl der Patienten, die postoperativ keine beziehungsweise weiterhin eine volle Kompressionsbehandlung durchführen. Die Werte sind in beiden Situationen mehr als doppelt so hoch, verglichen mit dem entsprechenden Wert des anderen Verfahrens (keine konservative Therapie: Omental; 36,36\% vs. Zervikal: 12,12\% sowie Omental; 18,18\% vs. Zervikal 51,51\%). Eine mögliche Erklärung für diese Ergebnisse könnte in der Herkunft der entsprechenden Lymphknoten liegen, die sich mit Kopf-Hals und Bauchregion deutlich unterscheiden.

Lymphknoten drainieren anatomisch unterschiedliche Geweberegionen. Sie gelten als primäre Interaktionsstelle zwischen Antigenen und immunologisch kompetenten Zellen des Körpers. Die strukturellen Veränderungen der Lymphknotenmorphologie spiegelt dadurch die lokale Immunreaktivität wieder (Cottier et al. 1973). Luscieti et al. fanden bereits in 1980 heraus, dass zervikale und mesenteriale Lymphknoten des Bauchraumes, 
aufgrund einer ständigen Exposition gegenüber äußeren Einflüssen, die höchste Anzahl an Keimzentren in ihren entsprechenden Lymphknoten zeigen (Luscieti et al. 1980). Eine Hypothese wäre, dass die omentalen Lymphknoten, aufgrund der unmittelbaren Nähe zum Magen, im Vergleich zu den supraklavikulären Lymphknoten direkter an immunologischen Prozessen beteiligt sind.

Dass die unterschiedlichen anatomischen Regionen entsprechend Einfluss auf den Lymphabfluss der Extremitäten haben können, zeigte bereits Gillies im Jahre 1935. Er legte bei an einem Lymphödem erkrankten Patienten eine künstliche Hautbrücke, mit Hilfe eines Hautlappens von Arm zu Bein oder von Arm zu Arm, an. Ein Ende der Hautbrücke lag im ödematösen Gewebe der betroffenen Extremität, das andere Ende im nächstgelegenen Lymphabflussgebiet. Nach einer gewissen Zeit wurde die Hautbrücke an ihrem einen Ende gelöst und der Hautlappen an der Empfängerregion eingelegt. Dem Autor nach zeigte sich in der Nachuntersuchung 15 Jahre nach erfolgter Intervention kein erneutes Auftreten eines Lymphödems (Gillies 1950; Withey et al. 2001).

Auch die von uns festgestellte Reduktionsrate der Erysipelneigung würde diese Hypothesen unterstützen. Prinzipiell haben die transplantierten Lymphknoten, mit ihren entsprechenden Lymphozyten und zur Phagozytose ausgestattete Makrophagen, die Möglichkeit Infektionen an ihrem neuen Wirkungsort zu bekämpfen (Sapountzis et al. 2014a). In unseren postoperativen Daten zeigte sich, mit einer Reduktion von $\mathrm{N}=3$ auf $\mathrm{N}$ $=0$ für die omentalen Lymphknoten und $\mathrm{N}=3$ auf $\mathrm{N}=1$ für die supraklavikulären Lymphknoten, ein vermindertes Auftreten von Erysipelen im entsprechenden Nachbeobachtungszeitraum.

Ob jedoch tatsächlich die Region der Spenderlymphknoten und ihre immunologische Aktivität die entscheidende Rolle für die besseren Ergebnisse spielt kann nicht sicher beurteilt werden.

Ein weiterer Grund könnte in der Anzahl der in den jeweiligen Transplantaten enthaltenen Lymphknoten liegen. Hypothetisch könnte davon ausgegangen werden, dass eine höhere Anzahl enthaltender Lymphknoten in den entsprechenden Transplantaten auch eine bessere Funktion versprechen würde. Schaverien charakterisierte in seinen anatomischen Studien den omentalen Lappen mit 6,4 \pm 7,3 Lymphknoten wohingegen im supraklavikulären Transplantat mit 3,3 \pm 1,5 deutlich weniger Lymphknoten vorhanden sind (Schaverien und Coroneos 2019). 
Die letzte potentielle Ursache könnte in der Anzahl der untersuchten Patienten liegen. Die zervikale Untersuchungsgruppe $(\mathrm{N}=33)$ stellt die dreifache Anzahl der Patienten dar. Rein theoretisch wäre es möglich, dass die ersten elf omentalen Lymphknotentransplantationen sehr gute Ergebnisse geliefert haben. Folgende 22 Patienten jetzt jedoch alle ohne Verbesserung in der konservativen Therapie verbleiben. Als Resultat wären die postoperativen Erhebungswerte der beiden Gruppen dann ziemlich ähnlich.

Zusammenfassend lässt sich sagen, dass die omentale Lymphknotentransplantation, in Bezug auf den Bedarf an konservativer Therapie, die besten Ergebnisse der drei untersuchten Verfahren liefert.

\subsection{Operationszeiten}

In der Abbildung 25 werden die drei Eingriffe im zeitlichen Vergleich aufgelistet. Es fällt auf, dass die zervikale Lymphknotentransplantation die mit Abstand längste aber auch eine der kürzesten Operationszeiten hat. Der Boxplot der omentalen Lymphknotentransplantationen ist am dichtesten, im Vergleich zu den anderen zwei Operationsverfahren. Somit hat die omentale Lymphknotentransplantation, mit einer Spannweite von 131 Minuten, die am wenigsten variierende Operationszeit (zervikale = 407 Minuten, Lymphgefäße = 285 Minuten). Auch die Mittelwerte der entsprechenden Eingriffe für omentale Lymphknoten mit 312,77 Minuten, zervikale Lymphknoten mit 340,66 Minuten und Lymphgefäßtransplantation mit 278,60 Minuten unterscheiden sich deutlich. Eine Erklärung soll in den unteren Abschnitten gefunden und diskutiert werden. 


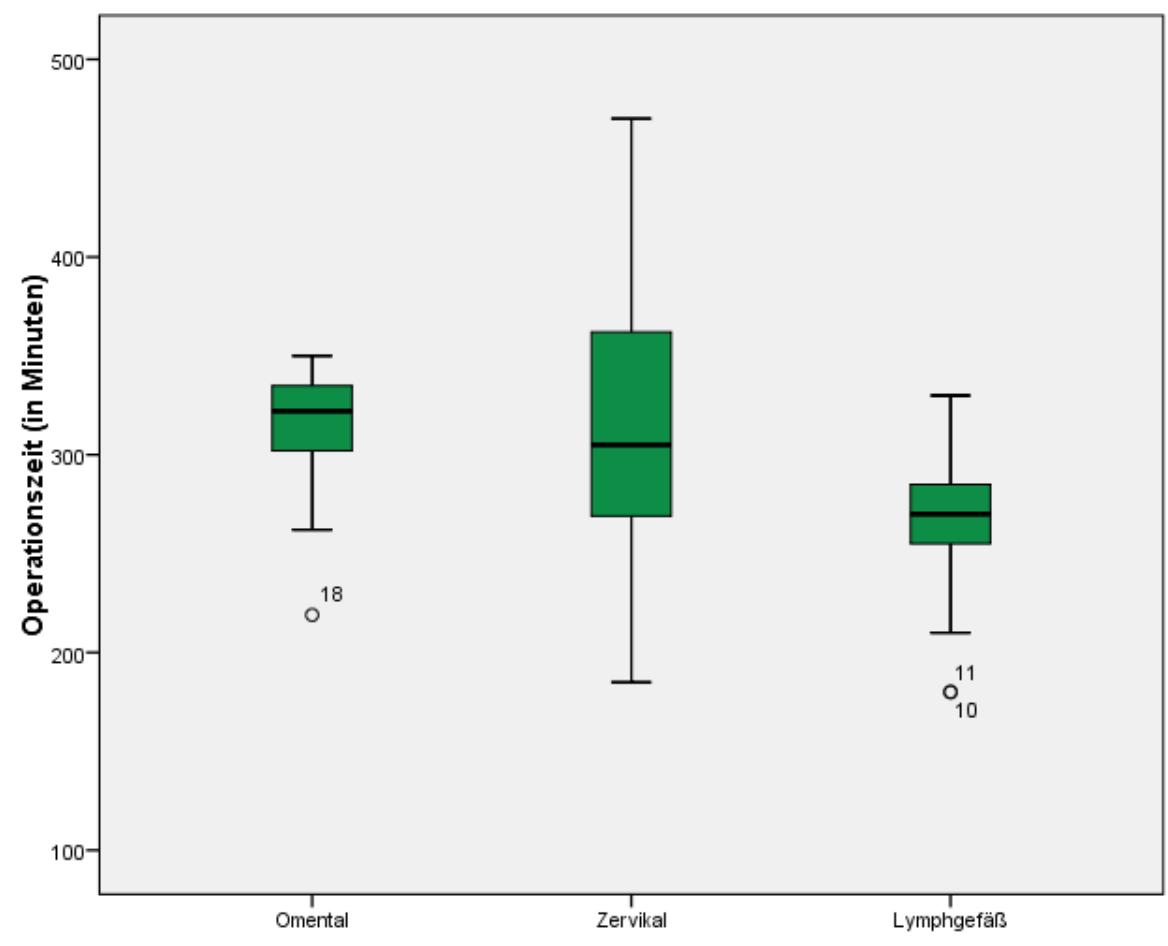

Abbildung 25: Die einzelnen Operationszeiten der jeweiligen Operationsmethode im zeitlichen Vergleich (jeweils Minimum, Maximum, Median und Ausreißer)

\subsubsection{Autologe omentale Lymphknotentransplantation roboterassistiert}

Logischerweise stellt die Etablierung beziehungsweise Durchführung eines neuen operativen Verfahrens immer auch eine besondere Herausforderung für Operateure, Assistenten und alle weiteren beteiligten Strukturen dar. Da wir im November 2017 erstmalig mit der omentalen roboterassistierten Lymphknotentransplantation begannen, wollten wir herausfinden, wie sich die Etablierung und der routinemäßige Umgang mit dem Operationsroboter auf den durchgeführten Eingriff auswirken. Hierfür bietet sich die benötigte Operationszeit als entsprechender Marker an. In der Effektstärke nach Cohen stellt unser Korrelationswert von $\mathrm{R}=-0,851$ für den roboterassistierten Teil der Operation einen großen Effekt dar. Unter Berücksichtigung der Tatsache, dass die Verwendung des Operationsroboters zu Untersuchungsbeginn im Jahr 2017 ein noch nicht etabliertes Verfahren an unserer Klinik beziehungsweise in unserer Abteilung darstellte, scheinen die erhobenen Korrelationswerte gut nachvollziehbar.

Nicht unerwähnt sollte hier blieben, dass für die erfolgreiche Durchführung des Eingriffs eine gute Kooperation mit den Kollegen der Allgemein- und Viszeralchirurgie der 
Universitätsmedizin Göttingen unabdingbar war. Auch hier wurde der Da-VinciOperationsroboter erst seit kürzerer Zeit standardmäßig für eine Vielzahl von Eingriffen eingesetzt. Die in dieser Zeit gemachten Erfahrungen, bezüglich der Anwendung des Robotersystems, stellte einen nicht unerheblichen Zugewinn auch für unsere Eingriffe dar. Deutlich wird dies bei Betrachtung des Korrelationskoeffizienten der Gesamtoperationszeit. Hier zeigt sich für $\mathrm{R}=-0,444$ ein deutlich geringerer Wert als für den reinen roboterassistierten Teil des Eingriffs. Somit lässt sich rückschließend feststellen, dass mit steigender Anzahl der Eingriffe eine Reduktion der Operationszeit, besonders für den roboterassistierten Eingriff, $\mathrm{zu}$ verzeichnen ist. Der weitaus geringere Korrelationskoeffizienz R für die Gesamtoperation lässt sich vermutlich mit der bereits vorhandenen Routine in der Präparation und Anastomiserung der vaskulären Strukturen erklären. Ein mathematisches und graphisches Korrelat liefern die entsprechenden Graphen in Abbilung 21 und Abbildung 22. Ein deutlich flacher abfallender Kurvenverlauf in der Abbildung 22 zeigt den geringeren Korrelationskoeffizient bei den Gesamteingriffszeiten.

Um eine bessere Annäherung an die in Zukunft wahrscheinliche Operationszeit zu bekommen, können entsprechende Operationswerte, mithilfe der Formel in Abbildung 21 und 22, berechnet werden. Bei einer verwendeten Anzahl von 30 beziehungsweise 60 Operationen ergeben sich die in Tabelle 11 dargestellte Werte.

Tabelle 11: Berechnete Näherung der erwarteten Operationszeiten

\begin{tabular}{|l|l|l|l|}
\hline & Funktion & $\mathbf{x}=\mathbf{3 0}$ (in Minuten) & $\mathbf{x}=\mathbf{6 0}$ (in Minuten) \\
\hline Gesamtoperationszeit & $y=351,04 x^{-0,06}$ & $y=286,23$ & $y=274,57$ \\
\hline Da-Vinci-Operationszeit & $y=202,23 x^{-0,263}$ & $y=82,67$ & $y=68,89$ \\
\hline
\end{tabular}

Durch den begrenzten Kurvenverlauf ergeben sich trotz Verdoppelung der Eingriffsanzahl nur noch eine geringe Änderung in den Operationszeiten. Vermutlich wird die tatsächliche Änderung der Operationszeit den oben genannten hypotetischen 60 Operationen entsprechen oder vielleicht sogar etwas darüber bleiben. Durch den Prozess des An- und Abdockens des Roboters sind bei jedem Eingriff bereits feste Zeiten vorgegeben, die sich auch im Verlauf in ihrer Dauer nicht weiter reduzierten lassen können.

Auf lange Sicht stellt die roboterassistierte Lymphknotentransplantation somit, im Bezug auf die benötigte Operationszeit, eine dem zervikalen Verfahren mindestens gleichwertige 
Alternative dar. Nach unseren erhobenen Daten und Berechungen ist die omentale Lymphknotentransplantation eine deutlich überlegenere Operationsmethode. Mit ihrer geringen Spannweite von 131 Minunten ist die Operation deutlich besser planbar und zeitlich extrem verlässlich. Bereits schon jetzt, mit duchgeführten Eingriffen von $\mathrm{N}=18$, waren sowohl Durchschnittswerte von 312,77 Minunten als auch die Medianwerte mit 322 Minuten besser beziehungsweise gleichwertig mit den entsprechenden Werte unseres zervikalen Lymphknotenkollektivs (Mittelwert: 340,66 Minuten; Medianwert: 322 Minuten).

In der aktuellen Vergleichsliteratur findet sich eine Publikation aus April 2020 (Frey et al. 2020). Hier wurden für fünf durchgeführte Eingriffe eine Operationszeit von neun Stunden und 19 Minuten als Durchschnittswert angegeben. Leider wird keine detaillierte Auflistung der einzelnen Operationsschritte beschrieben. Schlussendlich beginnt aber nach Setzen des Gefäßclips auf die $A$. gastroepiploica dextra die Ischämiezeit. Spätestens nach 120 Minuten sollten dann die Anastomosen angefertigt sein, um die Vitalität des Transplantates nicht zu gefährden. $\mathrm{Ob}$ der erste Schritt des Eingriffes, mit Präparation der Anschlussgefäße und roboterassistierter Hebung des Omentumlappens, bis zum Absetzen selbigen Lappens ungefähr sechs Stunden in Anspruch genommen haben könnte, eine weitere halbe Stunde wird erfahrungsgemäß für den Hautverschluss benötigt, kann abschließend nicht sicher beurteilt werden. Beachtlich bleibt jedoch die deutliche zeitliche Differenz zwischen unseren Operationszeiten und den oben genannten Werten. Eventuell kommen die längeren Zeiten durch die zusätzlich durchgeführte Flureszenzangiographie zu Stande. Ein möglicher Grund könnte aber auch die geringe Anzahl an Eingriffen und die damit verbundene geringe Routine sein. Zusätzlich ist $\mathrm{zu}$ erwähnen, dass die Transplantation teilweise in nichtanatomische Regionen durchgeführt wurde. Häufig ist hierbei der Hautverschuss mit der Deckung und dem Einpassen in die entsprechende Region deutlich aufwändiger. In der Axilla oder der Leiste lässt sich dagegen ein spannungsfreier Hautverschluss, nach zuvoriger Narbenlösung, problemlos durchführen. Ciudad et al. beschreiben in einem publizierten Fall die Lappenhebung mit einem Zeitwert von 55 Minuten. Weitere Zeitwerte der folgenden Operationsschritte werden hier nicht angegeben (Ciudad et al. 2016). Dieser angegebene Wert deckt sich aber ziemlich genau mit unserer beobachteten Konsolenzeit für die entsprechende Lappenhebung mit dem DaVinci.

\subsubsection{Zervikale Lymphknotentransplantation}

$\mathrm{Zu}$ ähnlicher Zeit wie die Lymphgefäßtransplantation wurde auch die Lymphknotentransplantation von C. Becker als mikrochirurgisches Verfahren geprägt 
(Becker et al. 2012; Becker et al. 2006; Becker et al. 1991). Vor Etablierung der omentalen Lymphknotenhebung mit dem Operationsroboter wurde in unserer Klinik von Januar 2013 bis Februar 2019 die Zervikalregion als Spenderstelle für entsprechende Lymphknotentransplantationen verwendet. Die Operationszeit der zervikalen Lymphknoten dient somit als direktes Gegenstück für die erhobenen Operationszeiten bei den roboterassistierten omentalen Lymphknoten.

In der Korrelationsanalyse zeigte sich der Korrelationskoeffizient $\mathrm{R}=0,083$. Somit kann in der Effektstärke nach Cohen kein Effekt zwischen Operationsanzahl und Operationszeit gefunden werden. Grafisch zeigt sich dies in Abbildung 23 mit einer Geraden ohne wesentliche Dynamik. Bei einem Mittelwert aller operativen Eingriffe von 340,66 Minuten und einem Median von 322 Minuten ist die besonders breite Spannweite mit 407 Minuten zu erwähnen. Ein Grund für diese extremen Werte liegt vermutlich an den feinen und variablen anatomischen Verhältnissen der supraklavikulären Region. Selbst mit Lupenbrillen ist die Lappenhebung, aufgrund der Nähe zum Plexus cervicalis sowie den Halsgefäßen, eine zeitaufwendige Arbeit, die sehr schwierig planbar und vorhersagbar ist.

\subsubsection{Lymphgefäßtransplantation}

Die autologe Lymphgefäßtransplantation wurde in den 1980er Jahren von Baumeister entwickelt und im laufe der Zeit in der rekonstruktiven Lymphchirurgie fest etabliert (Baumeister et al. 1981b; Baumeister et al. 1981a). In unserer Klinik wurden Patienten, vor allem in den Jahren 2011 bis 2015, in großer Zahl einer Lymphgefäßtransplantation zugeführt. Im restlichen Datenerhebungszeitraum von 2010 bis 2018 waren es jeweils zwei bis drei Eingriffe pro Jahr. Die Korrelationsanalyse der Operationszeiten aller Jahre zeigt für $\mathrm{R}=0,264$ einen geringen jedoch positiven Effekt. Somit nehmen die Operationszeiten, rein statistisch gesehen, mit steigender Anzahl auch zu. Dies wird auch an der postiven Trendlinie in Abbildung 24 nochmal grafisch dargestellt. Als möglicher Grund der positiven Korrelation kann beispielsweise ein Wechsel der Operateure vermutet werden. Eine zu beantwortende und die Operationszeit stark beeinflussende Frage ist hier auch, ob Hebestelle und Anschlussstelle parallel präpariert wurden oder, ob dies von einem Operateur nacheinander geschah. Es ist davon auszugehen, dass der Grund für die positive statistische Korrelation hier begründet liegt.

Die Lymphgefäßtransplantation bietet mit einem Mittelwert von 278,60 Minuten die geringste Gesamtoperationszeit. Wie die beiden anderen Verfahren gilt auch sie prinzipiell als mikrochirurgischer Eingriff und die Verwendung eines Operationsmikroskops und 
Lupenbrillen sind unabdingbar. Trotzdem ist sie, im Vergleich $\mathrm{zu}$ den anderen beiden Eingriffen, keine Operation unter Anwendung eines freien Lappens. Eine arterielle und venöse Gefäßnaht ist nicht notwendig, dafür müssen ein bis drei Lymphgefäßnähte angelegt werden. Nichtsdestotrotz entfallen die aufwendige Präparation des Lappens sowie dessen Vorbereitung.

\subsection{Schlussfolgerung}

Unsere Untersuchung mit ihren Ergebnissen zeigt deutlich, dass die autologe Lymphknotentransplantation sowie auch die autologe Lymphgefäßtransplantation vielversprechende Möglichkeiten in der Therapie der sekundären Extremitätenlymphödeme darstellt. In Zusammenschau der publizierten Artikel sowie unserer eigenen Erfahrungen mit der Erkrankung, stehen eine Vielzahl von Spenderstellen für die Entnahme der Lymphknoten zur Auswahl. Hierbei sollte eine minimale Hebedefektmorbidität angestrebt werden.

Unseren Ergebnissen nach stellt die roboterassistierte Lymphknotentransplantation aus dem Omentum die sicherste Methode bezüglich Hebedefektmorbiditäten dar. Im zeitlichen Vergleich zur suprakavikulären Spenderregion zeigen sich überlegene Werte. Zusätzlich waren die Ergebnisse bezüglich des Bedarfs an konservativer Therapie postoperativ deutlich besser.

\subsection{Limitationen}

Wie bereits unter 4.1.1 und unter 4.3 ausgeführt, zeigen unsere Auswertungen der konservativen Therapie postoperativ gute Ergebnisse, sodass einige Patienten entweder auf einen Teil, oder auf die komplette konservative Therapie verzichten konnten. Zusätzlich hatten viele unserer Patienten weite Anfahrtswege oder mussten in unserer Sprechstunde längere Wartezeiten in Kauf nehmen. Dies sollte bei Betrachtung der vermessenen Umfangswerte berücksichtigt werden.

Das Patientenkollektiv der supraklavikulären Lymphknoten und die Lymphgefäße stellen mit $\mathrm{N}=33$ sowie $\mathrm{N}=36$ eine in etwa vergleichbare Patientenanzahl dar. Unser omentales Lymphknotenkollektiv ist mit 18 bzw. elf Fällen deutlich geringer. Bessere Ergebnisse in der konservativen Therapie, die sich hier als vermeintliche Vorteile für eben dieses operative Verfahren zeigten, könnten eventuell bei gleichem Patientenkollektiv relativiert werden. Selbiges gilt für die Hebedefektmorbidität in unserer omentalen Lymph- 
knotengruppe. Die roboterassistierte Lymphknotentransplantation wird zum aktuellen Zeitpunkt von sehr wenigen Kliniken und Zentren weltweit durchgeführt. Dementsprechend ist die Datenlage aktuell auch noch sehr gering. Weitere Komplikationen, die momentan noch gar nicht bekannt sind, könnten in Zukunft deutlich relevanter werden. Allerdings wäre es auch möglich, dass sich unserer Ergebnisse mit zunehmendem Patientenkollektiv manifestieren und die Vorteile der omentalen Lymphknotentransplantation unterstreichen. 


\section{$5 \quad$ Zusammenfassung}

Das sekundäre Lymphödem der Extremitäten ist eine chronisch-progrediente Erkrankung des Lymphgefäßsystems, welches in der westlichen Welt überwiegend nach onkologischchirurgischer Therapie und Radiatio entsteht. Großer Leidensdruck und deutliche Einschränkungen in der Lebensqualität sind bezeichnend für diese, zum aktuellen Zeitpunkt, noch unheilbare Erkrankung. Durch den medizintechnischen Fortschritt in den letzten Jahren konnten vor allem mikrochirurgische Verfahren etabliert werden, die es ermöglichen Wege zu finden, um die Lymphabflusshindernisse zu umgehen und einen bestenfalls normalen Lymphabfluss wiederherzustellen.

Ziel dieser klinischen Arbeit war es unsere Ergebnisse, der seit November 2017 an unserer Klinik durchgeführten autologen omentalen Lymphknotentransplantation, darzustellen. In selbiger Arbeit sollten die Hebedefektmorbiditäten der anderen lymphchirurgischen Eingriffe herausgearbeitet werden und im Anschluss mit den aufgetretenen Komplikationen in unserem omentalen Lymphknotenkollektiv verglichen werden. Als lymphchirurgische Vergleichseingriffe wurden 33 autologe supraklavikuläre Lymphknotentransplantation und 36 autologe Lymphgefäßtransplantation nach Baumeister ausgewertet. Zusätzlich sollte darstellt werden, wie sich die Arbeit mit dem neu eingesetzten Da-Vinci-Operationsroboter über den Erhebungszeitraum verändert. Hierzu wurden die jeweiligen Operationszeiten erfasst und ausgewertet. Als weiteres Vergleichsmerkmal der drei Eingriffstypen, wurde der Bedarf an konservativer Therapie postoperativ herausgearbeitet.

Insgesamt bestand das Patientenkollektiv aus 87 beziehungsweise 80 Patienten, die sich im Erhebungszeitraum von März 2010 bis April 2020 im Schwerpunktbereich der Plastischen Chirurgie der Klinik für Unfallchirurgie, Orthopädie und Plastische Chirurgie der Universitätsmedizin Göttingen, aufgrund ihres sekundären Beinlymphödems, in Behandlung befanden. Die Umfangsmessungen unseres omentalen Lymphknotenkollektivs $(\mathrm{N}=11)$ erfolgte präoperativ sowie sechs und zwölf Monate postoperativ. Wie bereits erwartet, kam es zu keiner signifikanten Änderung der Umfangswerte des Gesamtkollektivs. Deutliche Unterschiede zeigten sich im Bezug auf Hebedefekte und dem Bedarf an postoperativer konservativer Therapie. Während in unserem omentalen Lymphknotenkollektiv bei einigen Patienten allenfalls ein leichtes abdominelles Spannungsgefühl, im Sinne einer Hebedefektmobidität, zu verzeichnen war, zeigte unser 
supraklavikuläres Lymphknotenkollektiv in der postoperativen Erhebung zehn Fälle von Hebedefektmorbiditäten bei einer Gesamtanzahl von 33 Operationen. Einmalig war eine chirurgische Intervention notwendig. In unserem Lymphgefäßkollektiv zeigten sich in 12 Fällen Hebedefektmorbiditäten. In einem Fall war eine operative Intervention notwendig. Das Maß an konservativer Therapie unterschied sich in den drei untersuchten Gruppen deutlich. Bei einem Gesamtkollektiv von 80 operierten Patienten konnten zehn Patienten $(12,5 \%)$ postoperativ (Nachbeobachtungszeitraum: Omentale LK $=18 \pm 3,48$ Monaten; Zervikale $\mathrm{LK}=14,15 \pm$ 4,9 Monaten; Lymphgefäßtx $=14,84 \pm$ 4,46 Monaten) auf die komplette konservative Therapie, bei klinisch stabilem Lymphödem, verzichten. Rechnerisch und in Relation zum jeweiligen Gesamtkollektiv entfielen auf die omentalen Lymphknoten $=36,36 \%$; Zervikalen Lymphknoten $=12,12 \%$ und auf die Lymphgefäße $=$ $5,55 \%$.

In Zusammenschau der Ergebnisse ist die omentale Lymphknotentransplantation, aufgrund der geringen Hebedefektmorbidität und der guten postoperativen Ergebnisse der supraklavikulären Lymphknotentransplantation deutlich überlegen. Auch in der Gegenüberstellung der Operationszeit lässt sich dies bestätigen.

Von besonderer Bedeutung für die Indikationsstellung einer Lymphgefäßtransplantation ist die fundierte Anamnese- und Befunderhebung. Aktuelle Studien haben hier zusätzliche therapeutische Möglichkeiten aufgezeigt, die sich als eventuelle Hilfstools der präoperativen Indikationsstellung und zur Reduktion von Hebedefektmorbiditäten in Zukunft als hilfreich erweisen könnten. 


\section{$6 \quad$ Literaturverzeichnis}

Ahmad G, O'Flynn H, Duffy JMN, Phillips K, Watson A (2012): Laparoscopic entry techniques. Cochrane Database Syst Rev 2 , CD006583

Alanen E, Lahtinen T, Nuutinen J (1999): Penetration of electromagnetic fields of an openended coaxial probe between $1 \mathrm{MHz}$ and $1 \mathrm{GHz}$ in dielectric skin measurements. Phys Med Biol 44, N169

Alemzadeh H, Raman J, Leveson N, Kalbarczyk Z, Iyer RK (2016): Adverse events in robotic surgery: A retrospective study of 14 years of FDA data. PLoS ONE $\underline{11}$, e0151470

Althubaiti GA, Crosby MA, Chang DW (2013): Vascularized supraclavicular lymph node transfer for lower extremity lymphedema treatment. Plast Reconstr Surg 131, 133e-135e

Alvin A, Diehl J, Lindsten J, Lodin A (1967): Lymph vessel hypoplasia and chromosome aberrations in 6 patients with Turner`s syndrome. Acta Derm Venereol, 25, 25-33

Aschen SZ, Farias-Eisner G, Cuzzone DA, Albano NJ, Ghanta S, Weitman ES, Ortega S, Mehrara BJ (2014): Lymph node transplantation results in spontaneous lymphatic reconnection and restoration of lymphatic flow. Plast Reconstr Surg 133, 301-310 Azzali G (1982): The ultrastructural basis of lipid transport in the absorbing lymphatic vessel. J Submicrosc Cytol 14, 45-54

Batista BN, Becker C, Germain M (2013): Vascularized lymph node transfer for patients with secondary inferior limb lymphedema. Plast Reconstr Surg 132, 32-33

Batista BN, Germain M, Faria JCM, Becker C (2017): Lymph node flap transfer for patients with secondary lower limb lymphedema. Microsurgery $\underline{37}, 29-33$

Baumeister RGH (1983): Mikrochirurgie des Lymphgefäßsystems. Chirurg 54, 374-378

Baumeister RGH (2009): Lymphödem und Lymphgefäßchirurgie. Gefässchirurgie $\underline{14}$, 401409

Baumeister RGH, Seifert J, Hahn D (1981a): Autotransplantation of lymphatic vessels. Lancet $\underline{1}, 147$

Baumeister RGH, Seifert J, Wiebecke B, Hahn D (1981b): Experimental basis and first application of clinical lymph vessel transplantation of secondary lymphedema. World J Surg $\underline{5}, 401-407$

Baumeister RGH, Siuda S, Bohmert H, Moser E (1986): A microsurgical method for reconstruction of interrupted lymphatic pathways: autologous lymph-vessel transplantation for treatment of lymphedemas. Scand J Plast Reconstr Surg 20, 141-146 
Baumeister RGH, Mayo W, Notohamiprodjo M, Wallmichrath J, Springer S, Frick A (2016): Microsurgical lymphatic vessel transplantation. J Reconstr Microsurg $\underline{32}$, 34-41

Bean JF, Hebal F, Hunter CJ (2015): A single center retrospective review of hair tourniquet syndrome and a proposed treatment algorithm. J Pediatr Surg 무, 1583-1585

Becker C (2016): Autologous Lymph Node Transfers. J Reconstr Microsurg 32, 28-33

Becker C, Hidden G, Godart S, Maurage H, Pecking A (1991): Free lymphatic transplant. Eur J Lymphol $\underline{6}, 75-80$

Becker C, Assouad J, Riquet M, Hidden G (2006): Postmastectomy lymphedema: longterm results following microsurgical lymph node transplantation. Ann Surg 243, 313-315

Becker C, Vasile JV, Levine JL, Batista BN, Studinger RM, Chen CM, Riquet M (2012): Microlymphatic surgery for the treatment of iatrogenic lymphedema. Clin Plast Surg $\underline{39}$, 385-398

Bellini C, Witte MH, Campisi C, Bonioli E, Boccardo F (2009): Congenital lymphatic dysplasias: genetics review and resources for the lymphologist. Lymphology 42, 36-41 Bennett CL, Stryker SJ, Ferreira, Adams J, Beart RW (1997): The learning curve for laparoscopic colorectal surgery. Preliminary results from a prospective analysis of 1194 laparoscopic-assisted colectomies. Arch Surg 132, 41-44

Bensley RP, Schermerhorn ML, Hurks R, Sachs T, Boyd CA, O'Malley AJ, Cotterill P, Landon BE (2013): Risk of late-onset adhesions and incisional hernia repairs after surgery. J Am Coll Surg 216, 1159-1167

Blaney JM, McCollum G, Lorimer J, Bradley J, Kennedy R, Rankin JP (2015): Prospective surveillance of breast cancer-related lymphoedema in the first-year post-surgery: feasibility and comparison of screening measures. Support Care Cancer 23, 1549-1559

Boggon RP, Palfrey AJ (1973): The microscopic anatomy of human lymphatic trunks. J Anat 114, 389-405

Bollinger A (1993): Microlymphatics of human skin. Int J Microcirc Clin Exp 12, 1-15

Bollinger A, Isenring G, Franzeck UK, Brunner U (1983): Aplasia of superficial lymphatic capillaries in hereditary and connatal lymphedema (Milroy's disease). Lymphology $\underline{16}, 27-$ 30

Borgstrom B, Laurell CB (1953): Studies of lymph and lymph-proteins during absorption of fat and saline by rats. Acta Physiol Scand 29, 264-280

Brandes R, Busse R: Kreislauf. In: Schmidt RF, Land F, Heckmann M (Hrsg.): Physiologie des Menschen. 31. Auflage; Springer, Heidelberg 2010, 572-626

Brauer WJ (1996): Lymphszintigraphie, Diagnostik mit dem Laufbandergometer. Lymphologie 20, 87-89 
Brauer WJ (2005): Fehlermöglichkeiten bei der Indikationsstellung, Durchführung und Interpretation der Funktionslymphszintigraphie. LymphForsch 모 85-90

Brauer WJ, Weissleder H (2017): Methodik und Ergebnisse der Funktionslymphszintigraphie: Erfahrungen bei 924 Patienten. Phlebologie 31, 118-125

Brice G, Child AH, Evans A, Bell R, Mansour S, Burnand K, Sarfarazi M, Jeffery S, Mortimer P (2005): Milroy disease and the VEGFR-3 mutation phenotype. J Med Genet $\underline{42}, 98-102$

Brorson H, Svensson H (1998): Liposuction combined with controlled compression therapy reduces arm lymphedema more effectively than controlled compression therapy alone. Plast Reconstr Surg 102, 1058-1068

Brorson H, Svensson H, Norrgren K, Thorsson O (1998): Liposuction reduces arm lymphedema without significantly altering the already impaired lymph transport. Lymphology $\underline{31}, 156-172$

Brorson H, Ohlin K, Olsson G, Svensson B, Svensson H (2008a): Controlled compression and liposuction treatment for lower extremity lymphedema. Lymphology 41, 52-63

Brorson H, Ohlin K, Svensson B (2008b): The facts about liposuction as a treatment for lymphoedema. J Lymphoedema $\underline{3}, 38$

Cambria RA, Gloviczki P, Naessens JM, Wahner HW (1993): Noninvasive evaluation of the lymphatic system with lymphoscintigraphy: a prospective, semiquantitative analysis in 386 extremities. J Vasc Surg 18, 773-782

Carlson JA (2014): Lymphedema and subclinical lymphostasis (microlymphedema) facilitate cutaneous infection, inflammatory dermatoses, and neoplasia: A locus minoris resistentiae.

Clin Dermatol 32, 599-615

Casley-Smith JR, Gaffney RM (1981): Excess plasma proteins as a cause of chronic inflammation and lymphodema: quantitative electron microscopy. J Pathol 133, 243-272

Casley-Smith JR, Clodius L, Piller NB (1980): Tissue changes in chronic experimental lymphoedema in dogs. Lymphology $\underline{13}, 130-141$

Charles RH: Elephantiasis Scroti: In: Latham AC, English TC (Hrsg.): A System of Treatment. Band 3; J \& A Churchill, London 1912, 504

Cheng MH, Huang JJ, Huang JJ, Nguyen DH, Saint-Cyr M, Zenn MR, Tan BK, Lee CL (2012): A novel approach to the treatment of lower extremity lymphedema by transferring a vascularized submental lymph node flap to the ankle. Gynecol Oncol 126, 93-98

Cheng MH, Chen SC, Henry SL, Tan BK, Lin MC-Y, Huang JJ (2013): Vascularized groin lymph node flap transfer for postmastectomy upper limb lymphedema: flap anatomy, recipient sites, and outcomes. Plast Reconstr Surg 131, 1286-1298 
Cheng MH, Huang JJ, Wu CW, Yang CY, Lin CY, Henry SL, Kolios L (2014): The mechanism of vascularized lymph node transfer for lymphedema: natural lymphaticovenous drainage. Plast Reconstr Surg $\underline{133}, 192 \mathrm{e}-8 \mathrm{e}$

Chlebicki MP, Oh CC (2014): Recurrent cellulitis: risk factors, etiology, pathogenesis and treatment. Curr Infect Dis Rep $\underline{16}, 422$

Ciudad P, Date S, Lee MH, Lo Torto F, Nicoli F, Araki J, Chen HC (2016): Robotic harvest of a right gastroepiploic lymph node flap. Arch Plast Surg 43, 210-212

Ciudad P, Manrique OJ, Date S, Sacak B, Chang WL, Kiranantawat K, Lim SY, Chen HC (2017a): A head-to-head comparison among donor site morbidity after vascularized lymph node transfer: Pearls and pitfalls of a 6-year single center experience. J Surg Oncol 115, 3742

Ciudad P, Maruccia M, Socas J, Lee MH, Chung KP, Constantinescu T, Kiranantawat K, Nicoli F, Sapountzis S, Yeo MSW (2017b): The laparoscopic right gastroepiploic lymph node flap transfer for upper and lower limb lymphedema: Technique and outcomes.

Microsurgery $\underline{37}, 197-205$

Cook KH, Park MC, Lee IJ, Lim SY, Jung YS (2016): Vascularized free lymph node flap transfer in advanced lymphedema patient after axillary lymph node dissection. J Breast Cancer 19, 92-95

Cornish BH (2006): Bioimpedance analysis: scientific background. Lymphat Res Biol 4, 4750

Cornish BH, Chapman M, Hirst C, Mirolo B, Bunce IH, Ward LC, Thomas BJ (2001): Early diagnosis of lymphedema using multiple frequency bioimpedance. Lymphology $\underline{34}$, $2-11$

Cottier H, Turk J, Sobin L (1973): A proposal for a standardized system of reporting human lymph node morphology in relation to immunological function. J Clin Pathol $\underline{26}$, $317-331$

Cozzaglio L, Coladonato M, Doci R, Travaglini P, Vizzotto L, Osio M, Gennari L (2008): Horner's Syndrome as a complication of thyroidectomy: Report of a case. Surg today $\underline{38}$, 1114-1116

Dale RF (1985): The inheritance of primary lymphoedema. J Med Genet 22, 274-278

Damstra RJ, Jagtman EA, Steijlen PM (2010): Cancer-related secondary lymphoedema due to cutaneous lymphangitis carcinomatosa: clinical presentations and review of literature. Eur J Cancer Care (Engl) 19, 669-675

Dimakakos E, Arkadopoulos N, Katsenis K, Toumpis S, Syrigos K (2009): Emmanuil Kondoleon: pioneer in surgical treatment for lymphedema. Lymphology 42, 42-45 
Dixon JB, Raghunathan S, Swartz MA (2009): A tissue-engineered model of the intestinal lacteal for evaluating lipid transport by lymphatics. Biotechnol Bioeng $\underline{103}$, 1224-1235

Drake RL, Vogl W, Mitchell AWM, Gray H (Hrsg.): Gray's anatomy for students. Churchill Livingstone/Elsevier, Philadelphia 2005

Dreyer G, Norões J, Figueredo-Silva J, Piessens WF (2000): Pathogenesis of lymphatic disease in bancroftian filariasis: a clinical perspective. Parasitol Today $\underline{16}, 544-548$

Dumanian GA, Futrell JW (1996): The Charles procedure: misquoted and misunderstood since 1950. Plast Reconstr Surg 무, 1258-1263

Dupuy A, Benchikhi H, Roujeau JC, Bernard P, Vaillant L, Chosidow O, Sassolas B, Guillaume JC, Grob JJ, Bastuji-Garin S (1999): Risk factors for erysipelas of the leg (cellulitis): case-control study. BMJ $\underline{318}, 1591-1594$

Dylke ES, McEntee MF, Schembri GP, Brennan PC, Bailey E, Ward LC, Kilbreath SL (2013): Reliability of a radiological grading system for dermal backflow in lymphoscintigraphy imaging. Acad Radiol 20, 758-763

Eberhardt RT, Raffetto JD (2014): Chronic venous insufficiency. Circulation 130, 333-346 Ehmke H: Das Kreislaufsystem. In: Pape H-C, Kurtz A, Silbernagl (Hrsg.): Physiologie. 7. Auflage; Georg Thieme Verlag, Stuttgart 2014, 213-265

Erdogan Iyigun Z, Selamoglu D, Alco G, Pilancı KN, Ordu C, Agacayak F, Elbüken F, Bozdogan A, Ilgun S, Guler Uysal F (2015): Bioelectrical impedance for detecting and monitoring lymphedema in patients with breast cancer. Preliminary results of the florence nightingale breast study group. Lymphat Res Biol $\underline{13}$, 40-45

Fátima Guerreiro Godoy M de, Pereira De Godoy JM (2015): Factitious lymphedema of the arm: case report and review of publications. Eur J Phys Rehabil Med 51, 337-339 Felmerer G, Sattler T, Lohrmann C, Tobbia D (2012): Treatment of various secondary lymphedemas by microsurgical lymph vessel transplantation. Microsurgery $\underline{32}, 171-177$ Felmerer G, Dowlatshahi AS, Stark GB, Földi E, Földi M, Ahls MG, Ströbel P, Aung T (2016): Lymphangiosarcoma: Is Stewart-Treves syndrome a preventable condition? Lymphat Res Biol 14, 35-39

Ferrell RE, Levinson KL, Esman JH, Kimak MA, Lawrence EC, Barmada MM, Finegold DN (1998): Hereditary lymphedema: evidence for linkage and genetic heterogeneity. Hum Mol Genet 7, 2073-2078

Földi E, Földi M, Weissleder H (1985): Conservative treatment of lymphoedema of the limbs. Angiology $\underline{36}, 171-180$ 
Földi E, Sauerwald A, Hennig B (2000): Effect of complex decongestive physiotherapy on gene expression for the inflammatory response in peripheral lymphedema. Lymphology $\underline{33}$, $19-23$

Földi E, Földi M, Clodius L, Neu H: Lymphostatische Krankheitsbilder. In: Földi M, Kubik S (Hrsg.): Lehrbuch der Lymphologie. 5. Auflage; Urban \& Fischer, München 2002, $253-348$

Földi M, Földi E: Physiologie und Pathophysiologie des Lymphgefäßsystems. In: Földi M, Kubik S (Hrsg.): Lehrbuch der Lymphologie. 5. Auflage; Urban \& Fischer, München 2002a, 209-252

Földi M, Földi E: Die akute Entzündung. In: Földi M, Kubik S (Hrsg.): Lehrbuch der Lymphologie. 5. Auflage; Urban \& Fischer, München 2002b, 443-448

Földi M, Strössenreuther RHK (Hrsg.): Foundations of manual lymph drainage. 3. Auflage; Elsevier Mosby, St. Louis 2004

Földi M, Clodius L, Casley-Smith JR: Das Lymphödem. In: Hornstein OP, Hundeiker M, Schönefeld J (Hrsg.): Neue Entwicklungen in der Dermatologie. Band 4; Springer, Berlin 1987, 107-122

Forte AJ, Boczar D, Huayllani MT, Lu X, McLaughlin SA (2019): Pharmacotherapy agents in lymphedema treatment: A systematic review. Cureus $\underline{11}$, e6300

Frey JD, Yu JW, Cohen SM, Zhao LC, Choi M, Levine JP (2020): Robotically assisted omentum flap harvest: A novel, minimally invasive approach for vascularized lymph node transfer. Plas Reconst Surg Glob Open $\underline{8}$, e2505

Fu MR, Axelrod D, Haber J (2008): Breast-cancer-related lymphedema: information, symptoms, and risk-reduction behaviors. J Nurs Scholarsh $\underline{40}$, 341-348

Fu MR, Chen CM, Haber J, Guth AA, Axelrod D (2010): The effect of providing information about lymphedema on the cognitive and symptom outcomes of breast cancer survivors. Ann Surg Oncol 17, 1847-1853

Füller J, Guderian D, Köhler C, Schneider A, Wendt TG (2008): Lymph edema of the lower extremities after lymphadenectomy and radiotherapy for cervical cancer. Strahlenther Onkol 184, 206-211

Gashev AA (2008): Lymphatic vessels: pressure- and flow-dependent regulatory reactions. Ann N Y Acad Sci 1131, 100-109

Gaw R, Box R, Cornish B (2011): Bioimpedance in the assessment of unilateral lymphedema of a limb: the optimal frequency. Lymphat Res Biol $\underline{9}$, 93-99

Giacalone G, Yamamoto T, Belva F, Hayashi A (2020): Bedside 3D visualization of lymphatic vessels with a handheld multispectral optoacoustic tomography device. J Clin Med $\underline{9}, 815$ 
Giannardi GF, Pelu G, Zampi G (1960): The clinical picture and histopathological basis of the so-called Stewart-Treves syndrome. Arch De Vecchi Anat Patol 34, 361-389

Gillies H (1950): The lymphatic wick. Proc R Soc Med 43, 1054-1056

Goldblum JR, Folpe AL, Weiss SW: Malignant vascular tumor. In: Goldblum JR, Folpe AL, Weiss SW (Hrsg.): Enzinger and Weiss's Soft Tissue Tumors. 6. Auflage;

Saunders/Elsevier, Philadelphia 2014, 733-732

Goldsmith HS (1974): Long term evaluation of omental transposition for chronic lymphedema. Ann Surg 180, 847-849

Goldsmith HS, los Santos R de (1967): Omental transposition in primary lymphedema. Surg Gynecol Obstet 125, 607-610

Goyal P, Chaudry G, Alomari AI: Conventional imaging modalities for the diagnosis of lymphedema. In: Greene AK, Slavin SA, Brorson H (Hrsg.): Lymphedema; Springer International Publishing, Cham 2015, 149-155

Guyton AC (1963): A concept of negative interstitial pressure based on pressures in implanted perforated capsules. Circ Res 12, 399-414

Guyton AC, Granger HJ, Taylor AE (1971): Interstitial fluid pressure. Physiol Rev $\underline{51}$, 527563

Hadjis NS, Carr DH, Banks L, Pflug JJ (1985): The role of CT in the diagnosis of primary lymphedema of the lower limb. AJR Am J Roentgenol 144, 361-364

Handley WS (1908): Lymphangioplasty: a new method for the relief of the brawny arm of breast-cancer and for similar conditions of lymphatic oedema. Lancet 171, 783-785

Handley WS (1910): Hunterian lectures on the surgery of the lymphatic system. Br Med J 1 , 853-864

Hara H, Mihara M, Hasegawa K, Yamanaka K (2016): Lymphatic dysfunction after ligation surgery for varicose vein. SAGE Open Med Case Rep ㄴ, 2050313X16672154

Harris SN, Ballantyne GH, Luther MA, Perrino AC (1996): Alterations of cardiovascular performance during laparoscopic colectomy: a combined hemodynamic and echocardiographic analysis. Anesth Analg $\underline{\text { 83 }}$, 482-487

Hasselhof V, Sperling A, Buttler K, Ströbel P, Becker J, Aung T, Felmerer G, Wilting J (2016): Morphological and Molecular Characterization of Human Dermal Lymphatic Collectors. PLoS ONE $\underline{11}$, e0164964

Hauck G (1972): Pathways between capillaries and lymphatics. Pflugers Arch $\underline{336}$, 55-57

Hauck G, Castenholz A (1992): Contribution of prelymphatic structures to lymph drainage. Z Lymphol 16, 6-9 
Hein HA, Joshi GP, Ramsay MA, Fox LG, Gawey BJ, Hellman CL, Arnold JC (1997): Hemodynamic changes during laparoscopic cholecystectomy in patients with severe cardiac disease. J Clin Anesth ㅁ, 261-265

Herpertz U: Ödeme und Lymphdrainage: Diagnose und Therapie von Ödemkrankheiten. 4. Auflage; Schattauer, Stuttgart 2010

Hidding JT, Beurskens CHG, van der Wees PJ, Bos WC, Nijhuis MW, van Laarhoven HWM, (2018): Changes in volume and incidence of lymphedema during and after treatment with docetaxel, doxorubicin, and cyclophosphamide (TAC) in patients with breast cancer. Support Care Cancer 26, 1383-1392

Hoffer EC, Meador CK, Simpson DC (1969): Correlation of whole-body impedance with total body water volume. J Appl Physiol 27, 531-534

Hu X, Zhang X, Gan H, Yu D, Sun W, Shi Z (2017): Horner syndrome as a postoperative complication after minimally invasive video-assisted thyroidectomy: A case report.

Medicine (Baltimore) $\underline{\text { 96, e } 8888}$

Illouz YG (1980): Une nouvelle technique pour les lipodystrophies localisées. Rev Chir Esth Franc 6, 9-14

Illouz YG (1983): Body contouring by lipolysis: a 5-year experience with over 3000 cases. Plast Reconstr Surg $\underline{72}, 591-597$

Inghammar M, Rasmussen M, Linder A (2014): Recurrent erysipelas-risk factors and clinical presentation. BMC Infect Dis $\underline{14}, 270$

International Society of Lymphology (ISL) (2013): The diagnosis and treatment of peripheral lymphedema: 2013 consensus document of the International Society of Lymphology. Lymphology 46, 1-11

Jain MS, Danoff JV, Paul SM (2010): Correlation between bioelectrical spectroscopy and perometry in assessment of upper extremity swelling. Lymphology $\underline{43}, 85-94$

Jansen FW, Kapiteyn K, Trimbos-Kemper T, Hermans J, Trimbos JB (1997): Complications of laparoscopy: a prospective multicentre observational study. Br J Obstet Gynaecol 104, 595-600

Jiang X, Anderson C, Schnatz PF (2012): The safety of direct trocar versus Veress needle for laparoscopic entry: a meta-analysis of randomized clinical trials. J Laparoendosc Adv Surg Tech A $\underline{22}, 362-370$

Johnson OW, Chick JFB, Chauhan NR, Fairchild AH, Fan C-M, Stecker MS, Killoran TP, Suzuki-Han A (2016): The thoracic duct: clinical importance, anatomic variation, imaging, and embolization. Eur Radiol 26, 2482-2493

Kaiserling E: Morphologische Alterationen. In: Földi M, Kubik S (Hrsg.): Lehrbuch der Lymphologie. 5. Auflage; Urban \& Fischer, München 2002, 349-398 
Karri V, Yang M-C, Lee IJ, Chen S-H, Hong JP, Xu E-S, Cruz-Vargas J, Chen H-C (2011): Optimizing outcome of charles procedure for chronic lower extremity lymphoedema. Ann Plast Surg $\underline{66}$, 393-402

Kasseroller RG (1998): The Vodder School: the Vodder method. Cancer 83, 2840-2842 Kaulesar Sukul DM, den Hoed PT, Johannes EJ, van Dolder R, Benda E (1993): Direct and indirect methods for the quantification of leg volume: comparison between water displacement volumetry, the disk model method and the frustum sign model method, using the correlation coefficient and the limits of agreement. J Biomed Eng 15, 477-480

Kinmonth JB, Wolfe JH (1980): Fibrosis in the lymph nodes in primary lymphoedema. Histological and clinical studies in 74 patients with lower-limb oedema. Ann R Coll Surg Engl 62, 344-354

Kinnaert P (1973): Anatomical variations of the cervical portion of the thoracic duct in man. J Anat $\underline{115}, 45-52$

Kirchhoff P, Dincler S, Buchmann P (2008): A multivariate analysis of potential risk factors for intra- and postoperative complications in 1316 elective laparoscopic colorectal procedures. Ann Surg 248, 259-265

Kiyonaga M, Mori H, Matsumoto S, Yamada Y, Sai M, Okada F (2012): Thoracic duct and cisterna chyli: evaluation with multidetector row CT. Br J Radiol 도, 1052-1058

Klingelhoefer E, Hesse K, Taeger CD, Prantl L, Stepniewski A, Felmerer G (2019): Factors affecting outcomes after supermicrosurgical lymphovenous anastomosis in a defined patient population. Clin Hemorheol and Microcirc $\underline{73}$, 53-63

Kondoleon E (1912a): Die chirurgische Behandlung der elephantiastischen Oedeme durch eine neue Methode der Lymphableitung. Münch Med Wschr 모, 2726-2729

Kondoleon E (1912b): Die operative Behandlung der elephantiastischen Oedeme. Zentralbl Chir $\underline{\text { 39, }}$ 1022-1025

Koshima I, Nanba Y, Tsutsui T, Takahashi Y, Itoh S (2003): Long-term follow-up after lymphaticovenular anastomosis for lymphedema in the leg. J Reconstr Microsurg 19 , 209215

Koshima I, Narushima M, Mihara M, Yamamoto T, Hara H, Ohshima A, Kikuchi K, Todokoro K, Seki Y, Iida T (2016): Lymphadiposal flaps and lymphaticovenular anastomoses for severe leg edema: Functional reconstruction for lymph drainage system. J

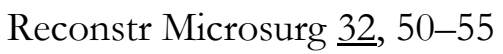

Kubik S: Anatomie des Lymphgefäßsystems. In: Földi M, Kubik S (Hrsg.): Lehrbuch der Lymphologie. 5. Auflage; Urban \& Fischer, München 2002, 1-178

Lahtinen T, Seppälä J, Viren T, Johansson K (2015): Experimental and analytical comparisons of tissue dielectric constant (TDC) and bioimpedance spectroscopy (BIS) in 
assessment of early arm lymphedema in breast cancer patients after axillary surgery and radiotherapy. Lymphat Res Biol 13, 176-185

Lanning P, Similä S, Suramo I, Paavilainen T (1978): Lymphatic abnormalities in Noonan's syndrome. Pediatr Radiol 7, 106-109

Lanz O (1911): Eröffnung neuer Abfuhrwege bei Stauung im Bauch und in den unteren Extremitäten. Zentralbl Chir $\underline{38}, 153$

Leak LV (1976): The structure of lymphatic capillaries in lymph formation. Fed Proc $\underline{35}$, 1863-1871

Lee M, McClure E, Reinertsen E, Granzow JW (2015): Lymphedema of the upper extremity following supraclavicular lymph node harvest. Plast Reconstr Surg 135, 1079e$1082 \mathrm{e}$

Lee MJ, Beith J, Ward L, Kilbreath S (2014): Lymphedema following taxane-based chemotherapy in women with early breast cancer. Lymphat Res Biol 12, 282-288 Leitlinie Lymphödem (2017): AWMF Reg.-Nr. 058-001. Hrsg. v. Gesellschaft Deutschsprachiger Lymphologen, Deutsche Gesellschaft für Lymphologie. Stand: 23.05.2017 https://www.awmf.org/uploads/tx_szleitlinien/058-

011_S2k_Diagnostik_und_Therapie_der_Lymphoedeme_2019-07.pdf; abgerufen am 20.09.2019

Lewis JM, Wald ER (1984): Lymphedema praecox. J Pediatr 104, 641-648

Lin C-H, Ali R, Chen SC, Wallace C, Chang YC, Chen HC, Cheng MH (2009): Vascularized groin lymph node transfer using the wrist as a recipient site for management of postmastectomy upper extremity lymphedema. Plast Reconstr Surg $\underline{123}$, 1265-1275

Lippi G, Favaloro EJ, Cervellin G (2012): Hemostatic properties of the lymph: relationships with occlusion and thrombosis. Semin Thromb Hemost $\underline{38}$, 213-221 Liu NF, Lu Q, Jiang ZH, Wang CG, Zhou JG (2009): Anatomic and functional evaluation of the lymphatics and lymph nodes in diagnosis of lymphatic circulation disorders with contrast magnetic resonance lymphangiography. J Vasc Surg 49, 980-987

Liu NF, Yan ZX, Wu XF (2012): Classification of lymphatic-system malformations in primary lymphoedema based on MR lymphangiography. Eur J Vasc Endovasc Surg 44, 345-349

Lohrmann C, Foeldi E, Speck O, Langer M (2006): High-resolution MR lymphangiography in patients with primary and secondary lymphedema. AJR Am J Roentgenol 187, 556-561 Lohrmann C, Pache G, Felmerer G, Foeldi E, Schaefer O, Langer M (2009): Posttraumatic edema of the lower extremities: evaluation of the lymphatic vessels with magnetic resonance lymphangiography. J Vasc Surg $\underline{49}, 417-423$ 
Lu Q, Xu J, Liu N (2010): Chronic lower extremity lymphedema: a comparative study of high-resolution interstitial MR lymphangiography and heavily T2-weighted MRI. Eur J Radiol $\underline{73}, 365-373$

Lüllmann-Rauch R: Taschenlehrbuch Histologie. 5. Auflage; Georg Thieme Verlag, Stuttgart 2015

Luscieti P, Hubschmid T, Cottier H, Hess MW, Sobin LH (1980): Human lymph node morphology as a function of age and site. J Clin Pathol $\underline{33}, 454-461$

Maerz DA, Beck LN, Sim AJ, Gainsburg DM (2017): Complications of robotic-assisted laparoscopic surgery distant from the surgical site. Br J Anaesth 118, 492-503

Magrina JF (2002): Complications of laparoscopic surgery. Clin Obstet Gynecol 4도, 469480

Maldonado AA, Chen R, Chang DW (2017): The use of supraclavicular free flap with vascularized lymph node transfer for treatment of lymphedema: A prospective study of 100 consecutive cases. J Surg Oncol $\underline{115}, 68-71$

Mansel RE, Fallowfield L, Kissin M, Goyal A, Newcombe RG, Dixon JM, Yiangou C, Horgan K, Bundred N, Monypenny I (2006): Randomized multicenter trial of sentinel node biopsy versus standard axillary treatment in operable breast cancer: the ALMANAC Trial. J Natl Cancer Inst $\underline{98}$, 599-609

Marsch WC (2005): The lymphatic system and the skin. Classification, clinical aspects und histology. Hautarzt 56, 277-293

Mayol J, Garcia-Aguilar J, Ortiz-Oshiro E, De-Diego Carmona JA, Fernandez-Represa JA (1997): Risks of the minimal access approach for laparoscopic surgery: multivariate analysis of morbidity related to umbilical trocar insertion. World J Surg 21, 529-533

Melsom H, Vaage S, Stenwig A, Solheim O (1981): Postmastectomy lymphangiosarcoma. Clinical features, diagnosis and therapy. Tidsskr Laegeforen 101, 951-953

Mihara M, Hara H, Araki J, Kikuchi K, Narushima M, Yamamoto T, Iida T, Yoshimatsu H, Murai N, Mitsui K (2012a): Indocyanine green (ICG) lymphography is superior to lymphoscintigraphy for diagnostic imaging of early lymphedema of the upper limbs. PLoS ONE 7, e38182

Mihara M, Hara H, Hayashi Y, Narushima M, Yamamoto T, Todokoro T, Iida T, Sawamoto N, Araki J, Kikuchi K (2012b): Pathological steps of cancer-related lymphedema: histological changes in the collecting lymphatic vessels after lymphadenectomy. PLoS ONE $\underline{7}$, e41126

Mikes DM, Cha BA, Dym CL, Baumgaertner J, Hartzog AG, Tacey AD, Calabria MR (1999): Bioelectrical impedance analysis revisited. Lymphology $\underline{32}$, 157-165 
Minari C, Cecconami L, Fioravanti A, Montemerani M, Scola C, Marcolongo R (1994): Lymphoedema of the limbs in rheumatoid arthritis. Clin Rheumatol 13, 464-469

Miranda Garcés M, Pons G, Mirapeix R, Masià J (2017): Intratissue lymphovenous communications in the mechanism of action of vascularized lymph node transfer. J Surg Oncol $115,27-31$

Mitsumori LM, McDonald ES, Wilson GJ, Neligan PC, Minoshima S, Maki JH (2015): MR lymphangiography: How i do it. J Magn Reson Imaging 42, 1465-1477

Molloy D, Kaloo PD, Cooper M, Nguyen TV (2002): Laparoscopic entry: a literature review and analysis of techniques and complications of primary port entry. Aust $\mathrm{N}$ Z J Obstet Gynaecol 42, 246-254

Mortimer PS (1998): The pathophysiology of lymphedema. Cancer $\underline{83}, 2798-2802$

Nguyen AT, Suami H (2015): Laparoscopic free omental lymphatic flap for the treatment of lymphedema. Plast Reconstr Surg 136, 114-118

Nicoli F, Constantinides J, Ciudad P, Sapountzis S, Kiranantawat K, Lazzeri D, Lim SY, Nicoli M, Chen P-Y, Yeo MS-W (2015): Free lymph node flap transfer and laser-assisted liposuction: a combined technique for the treatment of moderate upper limb lymphedema. Lasers Med Sci $\underline{30}, 1377-1385$

Notohamiprodjo M, Baumeister RGH, Jakobs TF, Bauner KU, Boehm HF, Horng A, Reiser MF, Glaser C, Herrmann KA (2009): MR-lymphangiography at 3.0 T- a feasibility study. Eur Radiol 19, 2771-2778

Nuutinen J, Ikäheimo R, Lahtinen T (2004): Validation of a new dielectric device to assess changes of tissue water in skin and subcutaneous fat. Physiol Meas $\underline{25}$, 447-454

Nuzzo G, Giuliante F, Tebala GD, Vellone M, Cavicchioni C (1997): Routine use of open technique in laparoscopic operations. J Am Coll Surg 184, 58-62

O'Brien BM, Khazanchi RK, Kumar PA, Dvir E, Pederson WC (1989): Liposuction in the treatment of lymphoedema; a preliminary report. Br J Plast Surg 42, 530-533

Ogata F, Narushima M, Mihara M, Azuma R, Morimoto Y, Koshima I (2007):

Intraoperative lymphography using indocyanine green dye for near-infrared fluorescence labeling in lymphedema. Ann Plast Surg 무, 180-184

Olszewski WL (2008): Contractility patterns of human leg lymphatics in various stages of obstructive lymphedema. Ann N Y Acad Sci 1131, 110-118

Olszewski WL, Engeset A (1980): Intrinsic contractility of prenodal lymph vessels and lymph flow in human leg. Am J Physiol 239, H775-H783 
Pabst R: Lymphknoten. In: Drenckhahn D (Hrsg.): Makroskopische Anatomie, Histologie, Embryologie, Zellbiologie. Band 2; 16. Auflage; Elsevier, Urban \& Fischer, München 2004, 156-159

Paskett ED, Naughton MJ, McCoy TP, Case LD, Abbott JM (2007): The epidemiology of arm and hand swelling in premenopausal breast cancer survivors. Cancer Epidemiol

Biomarkers Prev 16, 775-782

Pennock BE, Schwan HP (1969): Further observations on the electrical properties of hemoglobin-bound water. J Phys Chem $\underline{73}$, 2600-2610

Perry MD, Manoli A (2012): Reconstruction of the foot after leg or foot compartment syndrome. Crit Care Nurs Clin North Am 24, 311-322

Perugini RA, Callery MP: Complications of laparoscopic surgery. In: Holzheimer RG, Mannick JA (Hrsg.): Surgical Treatment: Evidence-Based and Problem-Oriented. Zuckschwerdt, München 2001

Pflug J, Calnan J (1968): The valves of the thoracic duct at the angulus venosus. Br J Surg 55, 911-916

Pittaluga P, Chastanet S (2012): Lymphatic complications after varicose veins surgery: risk factors and how to avoid them. Phlebology 27, 139-142

Pons G, Masia J, Loschi P, Nardulli ML, Duch J (2014): A case of donor-site lymphoedema after lymph node-superficial circumflex iliac artery perforator flap transfer. J Plast Reconstr Aesthet Surg 67, 119-123

Porter CJ (1997): Drug delivery to the lymphatic system. Crit Rev Ther Drug Carrier Syst $\underline{14}, 333-393$

Pschyrembel klinisches Wörterbuch: 267. Auflage; De Gruyter, Berlin 2017

Rabe E (1999): Artificial lymphedema from the clinical viewpoint. Wien Med Wochenschr $\underline{149}, 95$

Roozendaal R, Mebius RE, Kraal G (2008): The conduit system of the lymph node. Int Immunol 20, 1483-1487

Sacchi G, Weber E, Aglianò M, Raffaelli N, Comparini L (1997): The structure of superficial lymphatics in the human thigh: precollectors. Anat Rec 247, 53-62

Sapalidis K, Florou M, Tsopouridou K, Cheva A, Niki M, Pavlidis E, Koulouris C, Mantalovas S, Giannakidis D, Katsaounis A (2019): Horner's Syndrome: An uncommon complication of thyroidectomy and selective lateral neck dissection. Curr Health Sci J $\underline{45}$, $111-115$ 
Sapountzis S, Singhal D, Rashid A, Ciudad P, Meo D, Chen H-C (2014a): Lymph node flap based on the right transverse cervical artery as a donor site for lymph node transfer. Ann Plast Surg $\underline{73}, 398-401$

Sapountzis S, Ciudad P, Lim SY, Chilgar RM, Kiranantawat K, Nicoli F, Constantinides J, Wei MYS, Sönmez TT, Singhal D (2014b): Modified Charles procedure and lymph node flap transfer for advanced lower extremity lymphedema. Microsurgery $\underline{34}$, 439-447

Sawan S, Mugnai R, Lopes AB, Hughes A, Edmondson RJ (2009): Lower-limb lymphedema and vulval cancer: feasibility of prophylactic compression garments and validation of leg volume measurement. Int J Gynecol Cancer 19, 1649-1654

Schad H, Flowaczny H, Brechtelsbauer H, Birkenfeld G (1978): The significance of respiration for thoracic duct flow in relation to other driving forces of lymph flow. Pflugers Arch $\underline{378}, 121-125$

Schaverien MV, Coroneos CJ (2019): Surgical treatment of lymphedema. Plast Reconstr Surg 144, 738-758

Schmid-Schönbein GW (1990): Microlymphatics and lymph flow. Physiol Rev $\underline{70}$, $987-$ 1028

Schook CC, Mulliken JB, Fishman SJ, Grant FD, Zurakowski D, Greene AK (2011): Primary lymphedema: clinical features and management in 138 pediatric patients. Plast Reconstr Surg 127, 2419-2431

Sherman KA, Koelmeyer L (2011): The role of information sources and objective risk status on lymphedema risk-minimization behaviors in women recently diagnosed with breast cancer. Oncol Nurs Forum $\underline{38}$, E27-36

Silva AK, Chang DW (2016): Vascularized lymph node transfer and lymphovenous bypass: Novel treatment strategies for symptomatic lymphedema. J Surg Oncol 113, 932-939

Sixt M, Kanazawa N, Selg M, Samson T, Roos G, Reinhardt DP, Pabst R, Lutz MB, Sorokin L (2005): The conduit system transports soluble antigens from the afferent lymph to resident dendritic cells in the T cell area of the lymph node. Immunity 22, 19-29

Smeltzer DM, Stickler GB, Schirger A (1985): Primary lymphedema in children and adolescents: a follow-up study and review. Pediatrics $\underline{76}$, 206-218

Smith JB, McIntosh GH, Morris B (1970): The traffic of cells through tissues: a study of peripheral lymph in sheep. J Anat 107, 87-100

Smith ME, Riffat F, Jani P (2013): The surgical anatomy and clinical relevance of the neglected right lymphatic duct: review. J Laryngol Otol 127, 128-133

Smoot BJ, Wong JF, Dodd MJ (2011): Comparison of diagnostic accuracy of clinical measures of breast cancer-related lymphedema: area under the curve. Arch Phys Med Rehabil 2, 603-610 
Starling EH (1896): On the Absorption of Fluids from the Connective Tissue Spaces. J

Physiol 19, 312-326

Staverman AJ (1951): The theory of measurement of osmotic pressure. Rec Trav Chim, 344-352

Stepniewski A, Weiß SM, Felmerer G (2019): Die mikrochirurgische Therapie beim Beinlymphödem - Eine prospektive Studie. LymphForsch 1, 6-15

Stewart FW, Treves N (1948): Lymphangiosarcoma in postmastectomy lymphedema; a report of six cases in elephantiasis chirurgica. Cancer $\underline{1}, 64-81$

Stewart NJ, Pritchard DJ, Nascimento AG, Kang YK (1995): Lymphangiosarcoma following mastectomy. Clin Orthop Relat Res, 135-141

Strand SE, Persson BR (1979): Quantitative lymphoscintigraphy I: Basic concepts for optimal uptake of radiocolloids in the parasternal lymph nodes of rabbits. J Nucl Med $\underline{20}$, 1038-1046

Stüben BO, Klink CD, Krones CJ, Neumann UP (2015): Operative Versorgung von Trokarhernien. Meeting Abstract im Rahmen des 132. Kongress der Deutschen Gesellschaft für Chirurgie, München, 28.04.2015-01.05.2015

Stuchly MA, Stuchly SS (1980): Coaxial Line Reflection Methods for Measuring Dielectric Properties of Biological Substances at Radio and Microwave Frequencies-A Review. IEEE Trans. Instrum. Meas. 29, 176-183

Stutman LJ, Dumont AE, Shinowara GY (1965): Coagulation factors in human lymph and plasma. Am J Med Sci 250, 292-297

Sty JR, Boedecker RA, Scanlon GT, Babbitt DP (1979): Radionuclide "dermal backflow" in lymphatic obstruction. J Nucl Med 20, 905-906

Swaroop MN, Ferguson CM, Horick NK, Skolny MN, Miller CL, Jammallo LS, Brunelle CL, O'Toole JA, Isakoff SJ, Specht MC (2015): Impact of adjuvant taxane-based chemotherapy on development of breast cancer-related lymphedema: results from a large prospective cohort. Breast Cancer Res Treat 151, 393-403

Swartz MA (2001): The physiology of the lymphatic system. Adv Drug Deliv Rev $\underline{50}, 3-20$ Swartz MA, Berk DA, Jain RK (1996): Transport in lymphatic capillaries. I. Macroscopic measurements using residence time distribution theory. Am J Physiol 270, H324-9

Szuba A, Rockson SG (1998): Lymphedema: classification, diagnosis and therapy. Vasc Med $\underline{3}, 145-156$

Tiedjen KU, Heimann K-D, Knorz S: Radiologische Diagnostik bei Gliedmaßenschwellungen. In: Földi M, Kubik S (Hrsg.): Lehrbuch der Lymphologie. 5. Auflage; Urban \& Fischer, München 2002, 489-510 
Tobbia D, Semple J, Baker A, Dumont D, Johnston M (2009): Experimental assessment of autologous lymph node transplantation as treatment of postsurgical lymphedema. Plast Reconstr Surg 124, 777-786

Todd J, Austwick T, Berridge D, Tan LB, Barth JH (2011): B-type natriuretic peptide in lymphedema. Lymphology $\underline{44}, 29-34$

Trzewik J, Mallipattu SK, Artmann GM, Delano FA, Schmid-Schönbein GW (2001):

Evidence for a second valve system in lymphatics: endothelial microvalves. FASEB J $\underline{15}$, $1711-1717$

Tso P, Balint JA (1986): Formation and transport of chylomicrons by enterocytes to the lymphatics. Am J Physiol 250, G715-G726

Unno N, Inuzuka K, Suzuki M, Yamamoto N, Sagara D, Nishiyama M, Konno H (2007):

Preliminary experience with a novel fluorescence lymphography using indocyanine green in patients with secondary lymphedema. J Vasc Surg 또, 1016-1021

Unno N, Nishiyama M, Suzuki M, Tanaka H, Yamamoto N, Sagara D, Mano Y, Konno H (2010): A novel method of measuring human lymphatic pumping using indocyanine green

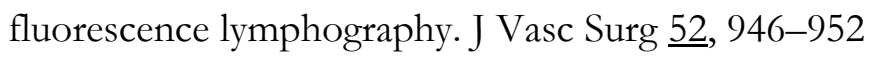

Vignes S, Blanchard M, Yannoutsos A, Arrault M (2013): Complications of autologous lymph-node transplantation for limb lymphoedema. Eur J Vasc Endovasc Surg 45, 516520

Vodder E (1936): Le drainage lymphatique, une nouvelle méthode thérapeutique. Santé Pour Tous, Paris 1936

Vodder E (1983): Die technische Grundlage der manuellen Lymphdrainge. Phys Ther $\underline{1}$, $17-18$

Warren AG, Janz BA, Slavin SA, Borud LJ (2007): The use of bioimpedance analysis to evaluate lymphedema. Ann Plast Surg $\underline{58}, 541-543$

Weinstein M, Roberts M (1950): Elephantiasis and the Kondoleon operation; a 20-year postoperative follow-up. Am J Surg 9ㅗ, 327-331

Weiss L: Lymphatic vessels and lymph nodes: In: Greep RO, Weiss L (Hrsg.): Histology. 3. Auflage; McGraw-Hill, New York 1973, 423-444

Weiss M, Baumeister RGH, Tatsch K, Hahn K (1996): Lymphoscintigraphy for noninvasive long term follow-up of functional outcome in patients with autologous lymph vessel transplantation. Nuklearmedizin $\underline{35}$, 236-242

Weiss M, Baumeister RGH, Hahn K (2003): Dynamic lymph flow imaging in patients with oedema of the lower limb for evaluation of the functional outcome after autologous lymph vessel transplantation: an 8-year follow-up study. Eur J Nucl Med Mol Imaging 30, $202-$ 
Weiss M, Burgard C, Baumeister R, Strobl F, Rominger A, Bartenstein P, Wallmichrath J, Frick A, Notohamiprodjo M (2014): Magnetic resonance imaging versus

lymphoscintigraphy for the assessment of focal lymphatic transport disorders of the lower limb: first experiences. Nuklearmedizin $\underline{53}, 190-196$

Welsch U, Deller T: Sobotta Lehrbuch Histologie. 3. Auflage; Elsevier, Urban \& Fischer, München 2010

Wiig H, Swartz MA (2012): Interstitial fluid and lymph formation and transport:

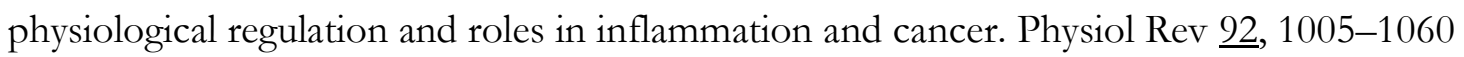

Willard-Mack CL (2006): Normal structure, function, and histology of lymph nodes. Toxicol Pathol 34, 409-424

Wilputte F, Renard M, Venner J-P, Strapart J, Leduc O, Leduc A, Klein P (2005):

Hemodynamic response to multilayered bandages dressed on a lower limb of patients with heart failure. Eur J Lymphol Relat Probl $\underline{15}, 1-4$

Winiwarter A (1892): Die chirurgischen Krankheiten der Haut und des Zellgewebes. In: Billroth CAT (Hrsg.): Deutsche Chirurgie. Band 23; Verlag Ferdinand Enke, Stuttgart 1892, 152-292

Withey S, Pracy P, Wood S, Rhys-Evans P (2001): The use of a lymphatic bridge in the management of head and neck lymphoedema. Br J Plast Surg $\underline{54}$, 716-719

Wohlrab J, Herrmann A, Marsch W (2000): Diagnostik und Therapie der Papillomatosis lymphostatica. LymphForsch 4, 61-68

World Health Organisation (1992): Lymphatic filariasis: the disease and its control. Fifth report of the WHO Expert Committee on Filariasis. World Health Organ Tech Rep Ser $\underline{821}, 1-71$

Yamamoto T, Matsuda N, Todokoro T, Yoshimatsu H, Narushima M, Mihara M, Uchida G, Koshima I (2011a): Lower extremity lymphedema index: a simple method for severity evaluation of lower extremity lymphedema. Ann Plast Surg 67, 637-640

Yamamoto T, Matsuda N, Doi K, Oshima A, Yoshimatsu H, Todokoro T, Ogata F, Mihara M, Narushima M, Iida T (2011b): The earliest finding of indocyanine green lymphography in asymptomatic limbs of lower extremity lymphedema patients secondary to cancer treatment: the modified dermal backflow stage and concept of subclinical lymphedema. Plast Reconstr Surg 128, 314e-321e

Yamamoto T, Yoshimatsu H, Narushima M, Yamamoto N, Hayashi A, Koshima I (2015): Indocyanine green lymphography findings in primary leg lymphedema. Eur J Vasc Endovasc Surg 49, 95-102

Yan A, Avraham T, Zampell JC, Aschen SZ, Mehrara BJ (2011): Mechanisms of lymphatic regeneration after tissue transfer. PLoS ONE $\underline{6}$, e17201 
Yu Y, Song Z, Xu Z, Ye X, Xue C, Li J, Bi H (2017): Bilayered negative-pressure wound therapy preventing leg incision morbidity in coronary artery bypass graft patients: A randomized controlled trial. Medicine (Baltimore) $\underline{96}$, e5925 


\section{Danksagung}

Ich möchte hiermit Herrn Prof. Dr. med. W. Lehmann, Direktor der Klinik für Unfallchirurgie, Orthopädie und Plastische Chirurgie, danken, der mir die Möglichkeit gegeben hat, diese Arbeit in seiner Klinik durchzuführen.

Ein besonderer Dank geht an Herrn Prof. Dr. med. G. Felmerer, Leiter des Schwerpunktbereiches Plastische Chirurgie, für die Überlassung des Themas, die gute Betreuung, die Überlassung von Literatur und Hilfsmaterialien sowie die stete Diskussionsund Hilfsbereitschaft.

Frau Dr. med. J. C. Witzel danke ich für die Überlassung wissenschaftlicher, statistischer Arbeitsmittel. 
